\title{
Acousto-Optic Scanners and Modulators
}

\section{Reeder N. Ward}

Noah Industries, Inc.

Melbourne, Florida, USA

\section{Mark T. Montgomery}

SkyCross, Inc.

Viera, Florida, USA

\section{Milton Gottlieb}

\section{Consultant}

Carnegie Mellon University

Pittsburgh, Pennsylvania, USA

\section{CONTENTS}

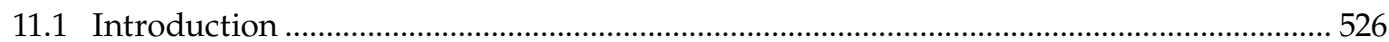

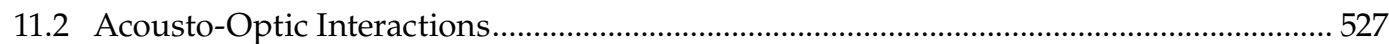

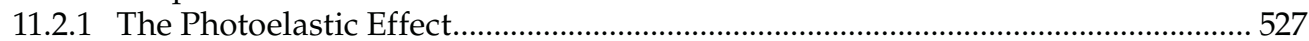

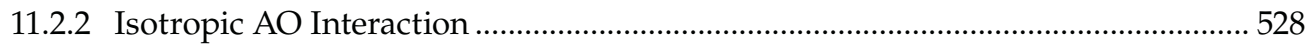

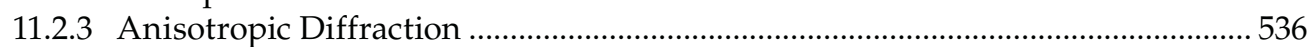

11.3 Acousto-Optic Modulator and Deflector Design ......................................................... 543

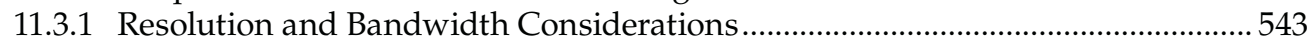

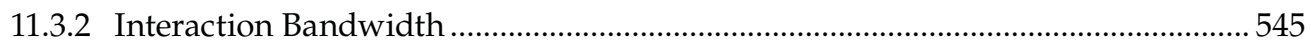

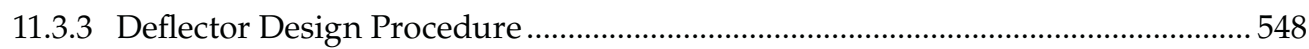

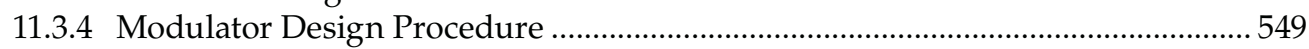

11.4 Specialized Acousto-Optic Devices for Scanning .......................................................... 551

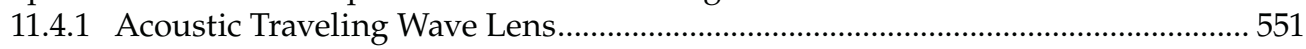

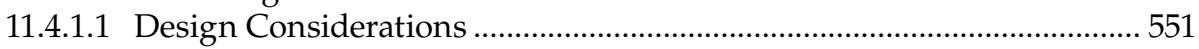

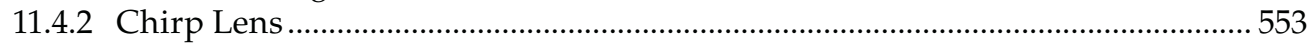

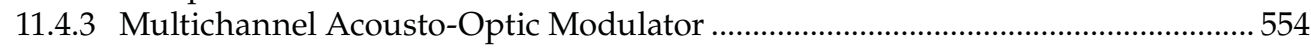

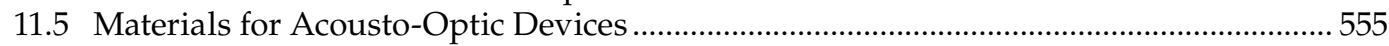

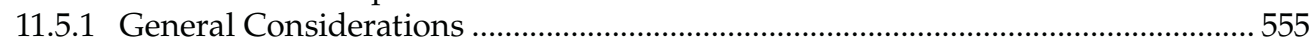

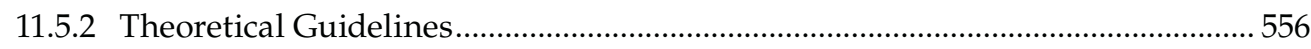

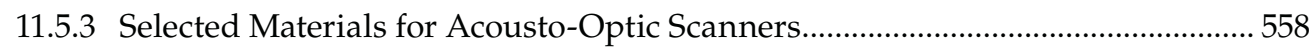

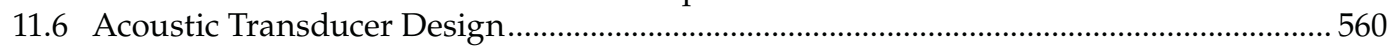

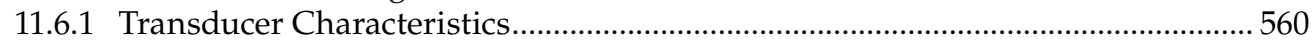

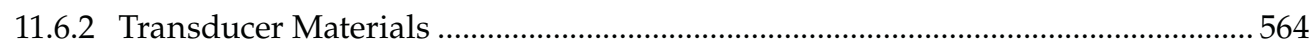

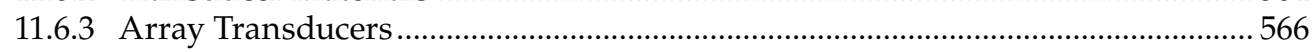

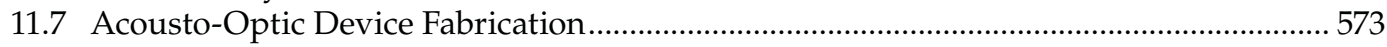

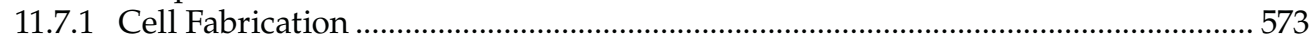

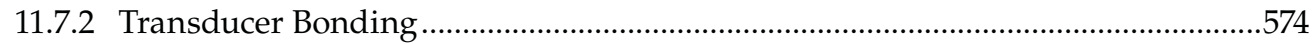

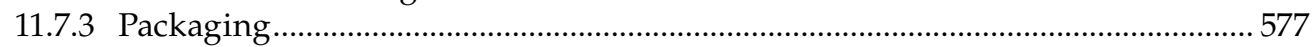

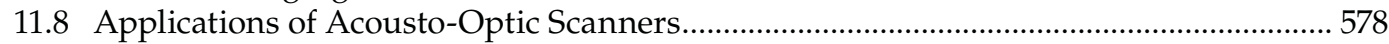

11.8.1 Multichannel Acousto-Optic Modulator for Polygonal Scanner ........................... 578

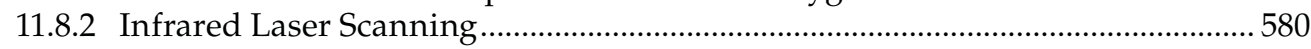




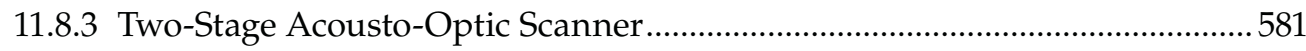

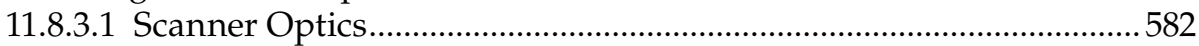

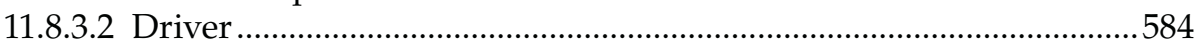

11.8.4 Applications of Acousto-Optic Devices and Acousto-Optic

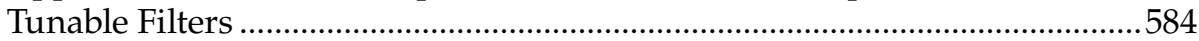

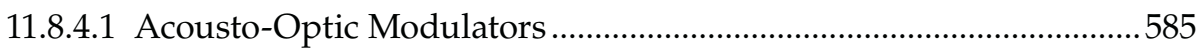

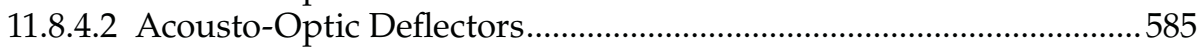

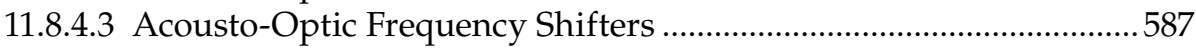

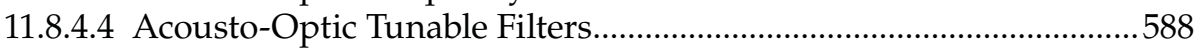

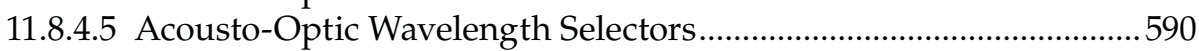

11.8.4.6 Polychromatic Acousto-Optic Modulators.......................................... 591

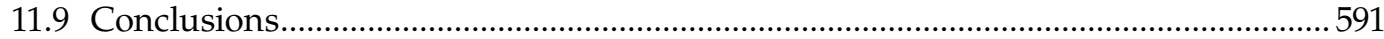

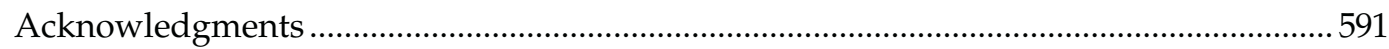

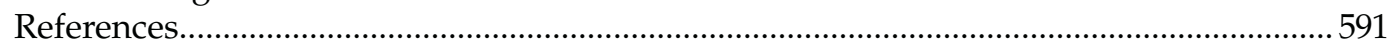

\subsection{INTRODUCTION}

It will be apparent to the reader of this book that there are a great variety of applications of lasers for which scanning devices are required, and that these applications include a wide range of performance requirements on the scanner. The basic specifications include speed, resolution, and random access time, and the choice of a scanner will be determined by these parameters. Acousto-optic (AO) scanners are best suited to those systems that are of moderate cost, since the cost of AO Bragg cells and the associated drive electronics are by no means trivial, and for which the resolution requirement is about 1000 spots. In addition, $\mathrm{AO}$ technology is most appropriate where random access times on the order of $10 \mu$ are needed, or where it may be desired to perform intensity modulation on the laser beam, as in image recording. There are currently many systems employing AO scanners, perhaps the most familiar being laser printers, in which the scanner capability is an excellent match to the system requirements. Large-area television display was one of the first applications considered for $\mathrm{AO}$ scanners, and it performs this function very well, although such display systems are relatively uncommon. These, as well as other applications of AO scanners, will be described in detail in a later section.

The interaction of light waves with sound waves has in recent years been the basis of a large number of devices related to various laser systems for display, information handling, optical signal processing, and numerous other applications requiring the spatial or temporal modulation of coherent light. The phenomena underlying these interactions were largely understood as long ago as the mid-1930s, but remained as scientific curiosities, having no practical significance, until the 1960s. During this period several technologies were developing rapidly, at the same time that many applications of the laser were being suggested that require high-speed, high-resolution scanning methods. These new technologies gave rise to high-efficiency, wideband acoustic transducers capable of operation to several gigahertz, high-power wideband solid-state amplifiers to drive such transducers, and the development of a number of new, synthetic AO crystals with very large figure of merit (low-drive-power requirements) and low acoustic losses at high frequencies. This combination of properties makes acousto-optics the method of choice for many systems, and is very often the only approach to satisfy demanding requirements. In this chapter, 
the underlying principles of $\mathrm{AO}$ interactions will be reviewed, and this will be followed by a description of the materials considerations and the relevant acoustic technology. AO scanning devices will be described in some detail, including the important features of optical design for various types of systems.

\subsection{ACOUSTO-OPTIC INTERACTIONS}

\subsubsection{The Photoelastic Effect}

The underlying mechanism of all AO interactions is very simply the change induced in the refractive index of an optical medium due to the presence of an acoustic wave. An acoustic wave is a traveling pressure disturbance that produces regions of compression and rarefaction in the material, and the refractive index is related to the density, for the case of an ideal gas, by the Lorentz-Lorenz relation

$$
\frac{n^{2}-1}{n^{2}+1}=\text { constant } \times r
$$

where $\eta$ is the refractive index and $\rho$ is the density. In fact, this relation is adhered to remarkably well for most simple solid materials as well. The elasto-optic coefficient is obtained directly by differentiation of Equation 11.1:

$$
r \frac{\partial n}{\partial r}=\frac{\left(n^{2}-1\right)\left(n^{2}+1\right)}{6 n}
$$

where it is understood that the derivative is taken under isentropic conditions. This is generally the case for ultrasonic waves, in which the flow of energy by thermal conduction is slow compared with the rate at which density changes within a volume smaller than an acoustic wavelength. The fundamental quantity given by Equation 11.2, also known as the photoelastic constant $p$, can be easily related to the pressure applied, with the result

$$
p=\frac{1}{b} \frac{\partial n}{\partial P}
$$

where $P$ is the applied pressure and $\beta$ is the compressibility of the material. The photoelastic constant of an ideal material with a refractive index of 1.5 is 0.59 . It will be seen later that the photoelastic constants of a wide variety of materials lie in the range from about 0.1 to 0.6 , so that this simple theory gives a reasonably good approximation to measured values.

The relation in Equation 11.3 follows from the usual definition of the photoelastic constant:

$$
\Delta\left(\frac{1}{e}\right)=\Delta\left(\frac{1}{n^{2}}\right)=p e
$$


where $\varepsilon$ is the dielectric constant $\left(\varepsilon=n^{2}\right)$ and $e$ is the strain amplitude produced by the acoustic wave. From Equation 11.4 it is easily seen that the change in refractive index, $\Delta n$, produced by the strain is

$$
\Delta n=-\frac{1}{2} n^{3} p e
$$

where $e$ is of the form $e_{0} \exp (i \Omega t)$ for an acoustic wave of frequency $\Omega$. The magnitude of refractive index change typical for $\mathrm{AO}$ devices is not large. Strain amplitudes lie in the range $10^{-8}$ to $10^{-5}$, so that using the above expressions for $\Delta n$ and $p$ gives for $\Delta n$ about $10^{-8}$ to $10^{-5}$ (for $n=1.5$ ). It may be somewhat surprising, then, that devices based upon such a small change in refractive index are capable of generating large effects, but it will be seen that this comes about because these devices are configured in a way that can produce large phase changes at optical wavelengths.

The relation defining the photoelastic interaction has been written in Equation 11.5 as a scalar relation, in which the photoelastic constant is independent of the directional properties of the material. In fact, even for an isotropic material such as glass, longitudinal acoustic waves and transverse (shear) acoustic waves cause the photoelastic interaction to assume different parameters. A complete description of the interaction, particularly for anisotropic materials, requires a tensor relation between the dielectric properties, the elastic strain, and the photoelastic coefficient. This may be represented by the tensor equation

$$
\Delta\left(\frac{1}{n^{2}}\right)_{i j}=\sum_{k l} p_{i j k l e_{k l}}
$$

where $\left(1 / n^{2}\right)_{i j}$ is a component of the optical index ellipsoid, $e_{k l}$ are the cartesian strain components, and $p_{i j k l}$ are the components of the photoelastic tensor. The crystal symmetry of any particular material determines which of the components of the photoelastic tensor may be nonzero, and also which components are related to others. This may be useful in determining whether some crystal, based only upon its symmetry, may even be considered for certain applications.

\subsubsection{Isotropic AO Interaction}

The most useful photoelastic effect is the ability of acoustic waves to diffract a light beam. There are several ways to understand how diffraction comes about; the acoustic wave may be thought of as a diffraction grating, made up of periodic changes in optical phase, rather than transparency, and moving at sonic velocity rather than being stationary. Thus, it is possible to analyze the diffraction as resulting from a moving phase grating. Alternatively, the light and sound may be thought of as particles, photons, and phonons, undergoing collisions in which energy and momentum are conserved. Either of these descriptions may be used to obtain all the important diffraction effects, but some are more easily understood on the basis of one or the other. It will be useful, then, to outline both of these approaches.

To examine the simplest case of plane acoustic waves interacting with plane light waves, consider Figure 11.1. Suppose the light wave, of frequency $\omega$ and wavelength $\lambda$, is incident from the left into a delay line with an acoustic wave of frequency $\Omega$ and wavelength $\Lambda$. If 


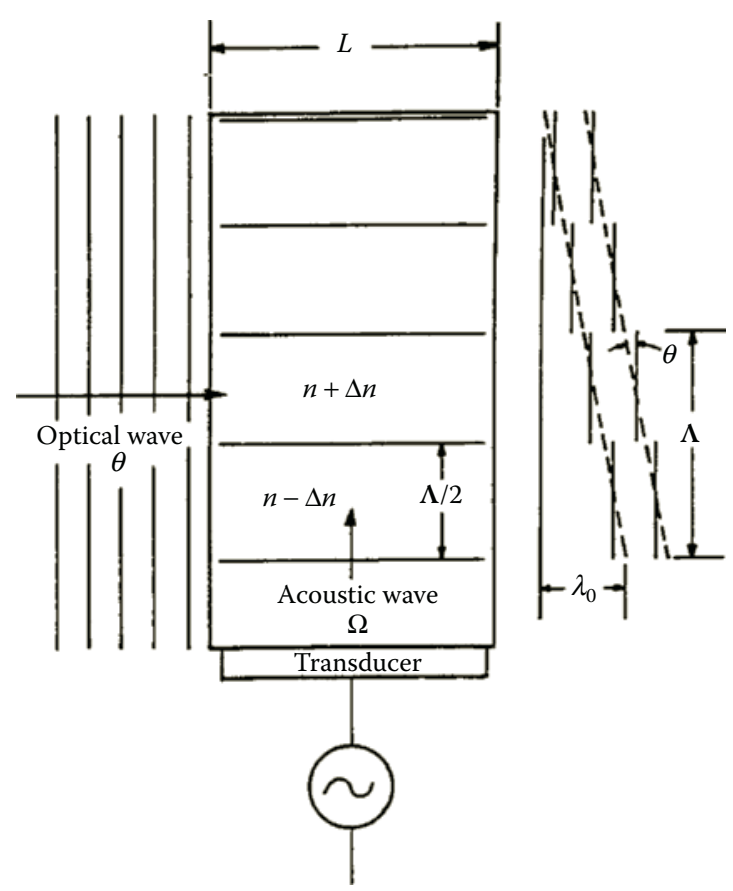

\section{FIGURE 11.1}

Tilting of optical wavefronts caused by upward-traveling acoustic wave.

the refractive index of the delay medium is $n+\Delta n$ in the presence of the acoustic wave, the phase of the optical wave will be changed by an amount

$$
\Delta f=2 p \frac{L}{I} \Delta n
$$

if the length of the delay line is $L$. Some typical values of $\Delta \phi$ can be obtained by assuming $L=2.5 \mathrm{~cm}(1 \mathrm{in})$ and $\lambda=0.5 \mu \mathrm{m}$, with $\Delta n$ reaching a peak value of $10^{-5}$. This yields a phase change of $\pi \mathrm{rad}$, which is, of course, quite large. It is large because $L / \lambda$, the number of optical wavelengths, is 50,000 , so that a very small $\Delta n$ can still produce a sizable $\Delta \phi$. If the electric field incident on the delay line is represented by

$$
E=E_{0} e^{i \omega t}
$$

then the field of the phase-modulated emerging light will be

$$
E=E_{0} e^{i(\omega t+\Delta f)}=e^{i \omega t} e^{i 2 p(L / 1)\left(a_{0} \sin \Omega t\right)}
$$

We shall not give a detailed derivation of the resulting temporal and spatial distribution of the light field, but we can use intuition and analogy with radiowave modulation to arrive at the resultant fields. It is well known from radio frequency (RF) engineering that the spectrum of a phase-modulated carrier of frequency $\omega$ consists of components separated by multiples of the modulation frequency $\Omega$, as shown in Figure 11.2. There is 


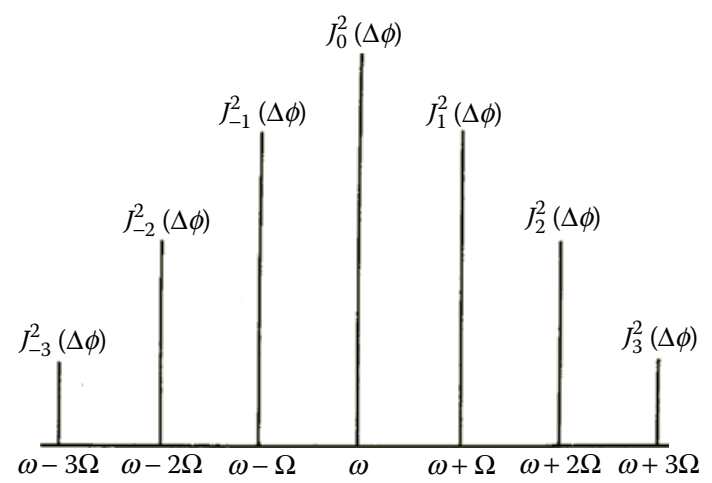

FIGURE 11.2

Intensity of diffracted orders due to Raman-Nath interaction.

a multiplicity of sidebands about the carrier frequency, such that the frequency of the $n$th sideband is $\omega+n \Omega$, where $n$ is both positive and negative. The amplitude of each sideband is proportional to the Bessel function of order equal to the sideband number, and whose argument is the modulation index $\Delta \phi$. Although not shown by Figure 11.2, note that the odd-numbered negative orders are $180^{\circ}$ out of phase with the others. The light emerging from the delay line is composed of a number of light waves whose frequencies have been shifted by $n \Omega$ from the frequency $\omega$ of the incident light. The relative amplitudes will be determined by the peak change in the refractive index.

In order to understand the diffraction of the light by the acoustic wave, consider the optical wavefronts in Figure 11.1. Because the velocity of light is about five orders of magnitude greater than the velocity of sound, it is a good approximation to assume that the acoustic wave is stationary in the time that it takes the optical wave to traverse the delay line. Suppose that during this instant the half-wavelength region labeled $\eta+\Delta n$ is under compression and $n-\Delta n$ is under rarefaction. Then the part of the optical plane wave passing through the compression will be slowed (relative to the undisturbed material of index n) while the part passing through the rarefaction will be speeded up. In this rough picture, the emerging wavefront will be "corrugated," so that if the corrugations are joined by a continuous plane its direction is tilted relative to that of the incident light wavefronts. Because the optical phase changes by $2 \pi$ for each acoustic wavelength $\Lambda$ along the acoustic beam direction, the tilt angle will be given by $\theta \cong \lambda / \Lambda$.

The direction normal to the tilted plane is the direction of optical power flow and represents the diffracted light beam. Note that the corrugated wavefront could just as well have been connected by a tilted wavefront at an angle given by $\theta \cong-\lambda / \Lambda$. This corresponds to the first negative order, the other to the first positive order. At this point we will note that an important consideration in the operation of AO Bragg cells, or for that matter of most ultrasonic devices, is the ratio of the acoustic wavelength $\Lambda$ to the transducer length $L$. The assumption that the acoustic energy propagates as a plane wave is valid when this ratio is very small or when there is little diffraction of the wave. However, when this ratio is not large, the acoustic propagation is more properly described in terms of the sum of plane waves, the angular spectrum of such plane waves increasing as the ratio increases. If we consider that partial wave which is propagating at an angle $\lambda / \Lambda$ to the forward direction, then we see that the light that has been diffracted into the first order may be diffracted a second time by this partial wave into an angle $2 \theta=2 \lambda / \Lambda$, and that the frequency of this light will once again be upshifted, for a total frequency shift of $2 \omega$. If the angular spectrum 
of acoustic waves contains sufficient power of still higher orders, then this process can be repeated again, so that light will be multiply diffracted into higher order angles, $\eta \theta=n \lambda / \Lambda$ each with a frequency shift $n \omega$. A similar argument holds for the negative orders, so that a complete set of diffracted light beams will appear as shown in Figure 11.3, where the angular deflection corresponding to the $n$th order is given by $\theta \eta \cong \pm \eta \lambda / \Lambda$ and the frequency of the light deflected into the $n$th order is $\omega \pm n \Omega$. The intensity of the carrier wave, or zeroth order, will be zero when the modulation index $\Delta \phi$ is equal to 2.4. The generally important first order will have a maximum value of $34 \%$ of the input for $\Delta \phi=1.8$, decreasing for higher modulation. These phenomena were described by Debye and Sears ${ }^{1}$ and are often referred to as Debye-Sears diffraction. Similar observations were published almost simultaneously by Lucas and Biquard. ${ }^{2}$ An extensive theoretical analysis of the effect was given by Raman and Nath, ${ }^{3}$ and so it is alternatively referred to as Raman-Nath diffraction. A distinctive feature of this type of diffraction is that it is limited to low acoustic frequencies (or relatively long wavelengths). The origin of this limitation lies in the diffraction spreading of the light beam as it traverses apertures formed by the columns of compression and rarefaction in the acoustic beam. If the length of the acoustic beam along the light propagation direction is large enough, the diffraction spread of the light between adjacent compression and rarefaction regions will overlap, so that the Debye-Sears model is no longer valid. To estimate the characteristic length $L_{0}$ that bounds the Debye-Sears model, suppose the compression and rarefaction apertures are one-half an acoustic wavelength, $\Lambda / 2$, so that the angular diffraction spread of the light is $\delta \phi \approx 2 \lambda / \Lambda$. Then $L_{0}$ can be defined as that interaction length for which the aperture diffraction spreads the light by one-half an acoustic wavelength,

$$
L_{0} d f=\frac{\Lambda}{2}
$$

or

$$
L_{0}=\frac{\Lambda^{2}}{41}
$$

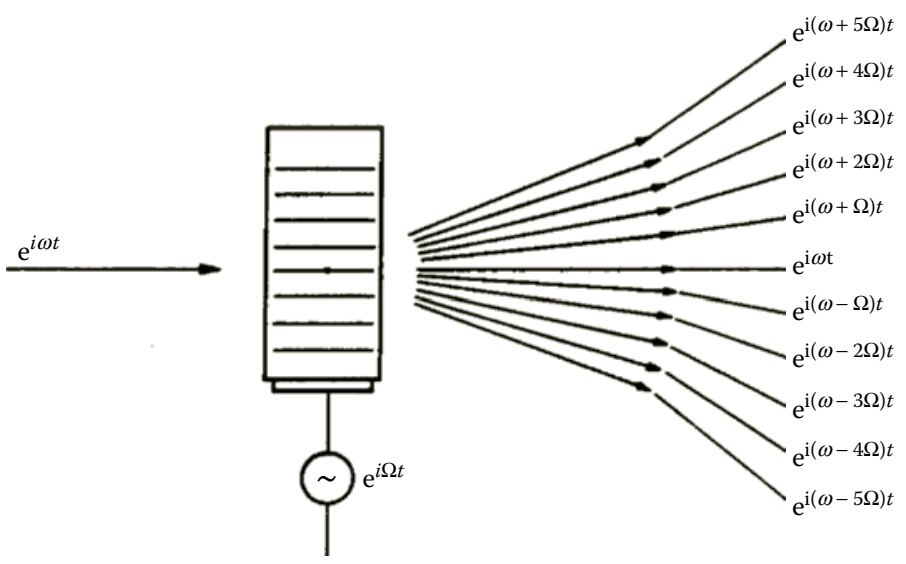

FIGURE 11.3

Raman-Nath diffraction of light into multiple orders. 
The interaction length is sometimes expressed in terms of a ratio known as the RamanNath parameter (often referred to as the Klein-Cook parameter) $Q$, as

$$
Q=\frac{2 p L I}{\Lambda^{2}}
$$

Devices with interaction length $Q<\pi$ are said to operate in the Raman-Nath regime, while devices with $Q>4 \pi$ are said to operate in the Bragg regime. For typical values of $L=1 \mathrm{~cm}$ and $\lambda=6.33 \times 10^{-5} \mathrm{~cm}, Q=1$ for $\Lambda=0.0159 \mathrm{~cm}(0.006 \mathrm{in})$, which corresponds to a frequency of $31.4 \mathrm{MHz}$ for a material whose acoustic velocity is $5 \times 10^{5} \mathrm{~cm} / \mathrm{s}\left(2 \times 10^{5} \mathrm{in} / \mathrm{s}\right)$.

In the Bragg regime, the thin grating approximation no longer holds. If the incident light beam is normal to the sound beam propagation direction, the higher diffraction orders interfere destructively beyond $L_{0}$, eventually completely wiping out the diffraction pattern. In order for constructive interference to take place, the angle of incidence must be tilted with respect to the acoustic beam direction. To better understand what conditions must be satisfied for this, it is easier to think of the light and sound waves as colliding particles, photons, and phonons. In this description, the light and sound take on the attributes of particles, and the dynamics of their collisions are governed by the laws of conservation of energy and conservation of momentum. The magnitudes of the momenta of the light and sound waves are given by the well-known expressions

$$
|k|=\frac{w n}{c}=\frac{2 p n}{I_{0}}
$$

and

$$
|K|=\frac{\Omega}{u}=\frac{2 p}{\Lambda}
$$

respectively. In the latter equation, $\nu$ is the velocity of sound in the delay medium, $v=$ $2 \pi \Omega \Lambda$. Conservation of momentum is expressed by the vector relation

$$
k_{i}+K=k_{d}
$$

the diagram for which is shown in Figure 11.4a, where $k_{i}$ and $k_{d}$ represent the momentum of the incident photon and the diffracted photon, respectively. The process may be thought of as one in which the acoustic phonon is absorbed by the incident photon to form the diffracted photon. Thus, conservation of energy requires that

$$
h w_{0}=h w_{1}+h \Omega
$$

or

$$
w_{d}=w_{1}+\Omega
$$

in which $h$ is Planck's constant. 
(a)

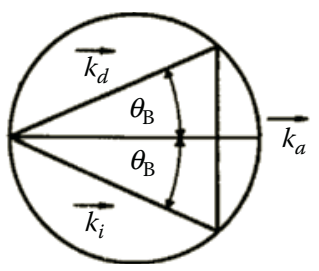

(b)

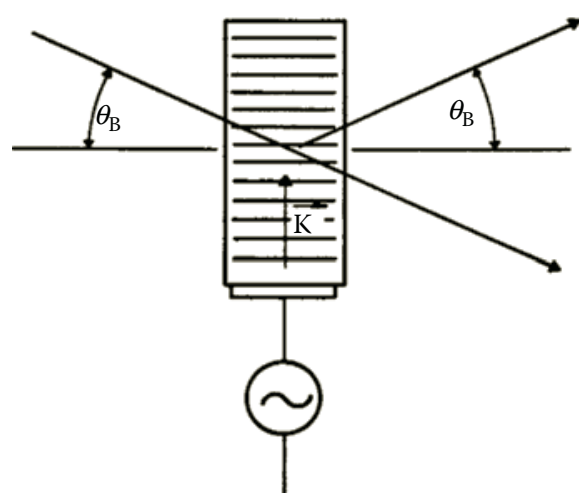

FIGURE 11.4

Bragg diffraction in isotropic medium: (a) phasor diagram; (b) Bragg cell deflection.

Because $\omega_{i}$ lies in the optical frequency range, and $\Omega$ will typically lie in the RF or microwave range, $\Omega<<\omega_{i}$ so that $\omega_{d} \approx \omega_{i}$. This results in the magnitudes of $k_{i}$ and $k_{d}$ being almost equal, so that the momentum triangle of Figure 11.4a is isosceles, and the angle of incidence (with respect to the normal to $K$ ) is equal to the angle of diffraction. This angle of incidence is easily obtained from Figure 11.4a as

$$
\sin q_{B}=\frac{1}{2} \frac{K}{k}=\frac{1}{2} \frac{1}{\Lambda}
$$

It is called the Bragg angle because of its similarity to the angle of diffraction of X-rays from the regularly spaced planes of atoms in crystals. The configuration of these vectors in relation to the delay line is shown in Figure 11.4b. In order for diffraction to take place, the light must be incident at the angle $\theta_{B}$, and the diffracted beam will appear only at this same angle. In contrast to the Debye-Sears regime, there are no higher-order diffracted beams. In the full mathematical treatment of the Bragg limit $(Q \gg>1)$, light energy may appear at the higher orders, but the probability of its doing so is extremely small so that the intensity at higher orders is essentially zero. The diagrams in Figure 11.4 show the interaction in which the diffracted photon is higher in energy than that of the incident photon, but the reverse can also take place. If the sense of the vector $K$ is reversed with respect to $k_{i}$, then $\omega_{d}=\omega_{i}-\Omega$, and the diffracted negative first-order results.

It is important to understand that the Debye-Sears effect and Bragg diffraction are not different phenomena, but are the limits of the same mechanism. The Raman-Nath parameter $Q$ determines which is the appropriate limit for a given set of values $\lambda, \Lambda$, and $L$. Quite commonly in practice, these values will be chosen such that neither limit applies, and $Q \approx$ 1. In this case, the mathematical treatment is quite complex, and experimentally it is found that one of the two first-order diffracted beams may be favored, but that higher orders will be present.

Having obtained the angular behavior of light diffracted by acoustic waves, the next most important characteristic is the intensity of the diffracted beam. Again, the full mathematical treatment is beyond the scope of this book, but a very good intuitive calculation leads to results that are useful. Referring to the spectrum of a phase-modulated wave 
shown in Figure 11.2, we can see that the ratio of the intensity in the first order to that in the zero order is

$$
\frac{I_{1}}{I_{0}}=\left[\frac{J_{1}(\Delta f)}{J_{0}(\Delta f)}\right]^{2}
$$

We shall now show in detail how this result comes about for acousto-optically diffracted light. The acoustic power flow is given by

$$
p=\frac{1}{2} c u e^{2}
$$

where $c$ is the elastic stiffness constant. The elastic stiffness constant is related to the bulk modulus $\beta$ and the density and acoustic velocity through the well-known expression

$$
c=\frac{1}{b} r u^{2}
$$

Thus, the acoustic power density is

$$
P_{A}=\frac{1}{2} r u^{3} e^{2}
$$

We can express the phase modulation depth in terms of the acoustic power density, using Equation 11.5 for $\Delta n$ and Equation 11.7 for $\Delta \phi$, with the result

$$
\Delta f=2 p \frac{L}{I} \Delta n=-p \frac{L}{I} n^{3} p\left(\frac{2 P_{A}}{r u^{3}}\right)^{1 / 2}
$$

For small modulation index, the zero-order and first-order Bessel functions can be approximated by

$$
J_{0}(\Delta f) \approx \cos (\Delta f) \approx 1-\Delta f
$$

and

$$
J_{1}(\Delta f) \approx \sin (\Delta f) \approx \Delta f
$$

so that the small-signal approximation to the diffracted light is, from Equation 11.17,

$$
\frac{I_{1}}{I_{0}} \approx(\Delta f)^{2}=\frac{p^{2}}{2}\left(\frac{L}{I}\right)^{2}\left(\frac{n^{6} p^{2}}{r u^{3}}\right) P_{A}
$$


This efficiency may be expressed in terms of the total acoustic power $P$,

$$
P=P_{A}(L H)
$$

where $H$ is the height of the transducer, and

$$
\frac{I_{1}}{I_{0}}=\frac{p^{2}}{2} \frac{L}{H}\left(\frac{n^{6} p^{2}}{r u^{3}}\right) \frac{P}{I^{2}}
$$

The quantity in parentheses depends only upon the intrinsic properties of the AO material, while the other parameters depend upon external factors. It is therefore defined as the figure of merit of the material,

$$
M_{2}=\left(\frac{n^{6} p^{2}}{r u^{3}}\right)
$$

from which it can be seen that, in general, the most important factors leading to high AO efficiency will be a high refractive index and a low acoustic velocity. This does not guarantee a large figure of merit, since the photoelastic constant may be very small, or even zero.

The other factors in Equation 11.25 have the following effect on the diffraction efficiency. The efficiency decreases quadratically with increasing wavelength, so that the power requirements for operation in the infrared (IR) may be hundreds of times that required for the visible. For high efficiency, it will be desirable to have a large aspect ratio, $L / H$, leading to a configuration as shown in Figure 11.5. It is difficult to make conventional bulk devices with $H$ much less than $1 \mathrm{~mm}$, so that aspect ratios up to about 50 can be achieved. Much higher aspect ratios can be reached in guided optical wave devices. A more exact calculation of the diffraction efficiency in the Bragg regime ${ }^{4}$ yields the result

$$
\frac{I_{1}}{I_{0}}=\sin ^{2}\left[\frac{p^{2}}{2} \frac{L}{H} M_{2} \frac{P}{I^{2}}\right]^{1 / 2}
$$

For low signal levels Equation 11.27 reduces to the same expression as in Equation 11.25. To obtain an order of magnitude for the power requirements of an acousto-optic deflector

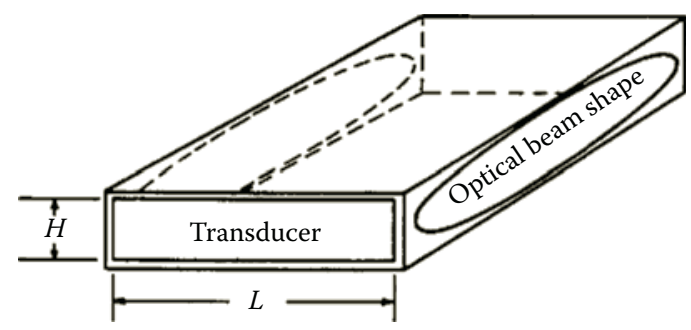

FIGURE 11.5

Transducer and optical beam shapes for optimization of acousto-optic diffraction. 
(AOD), let us assume a material with $n=1.5, \rho=3, \nu=5 \times 10^{5} \mathrm{~cm} / \mathrm{s}$, and a photoelastic constant calculated from the $\simeq$ Lorentz-Lorenz expression, $p \cong 0.6$, so that $M \simeq 1.1 \times 10^{-17} \mathrm{~s}^{3} / \mathrm{g}$. If the remaining parameters are $L=1 \mathrm{~cm}$ and $\lambda=0.6 \mu \mathrm{m}$, then by assuming a maximum acoustic power density for $\mathrm{CW}$ operation of $1 \mathrm{~W} / \mathrm{cm}^{2}\left(10^{7} \mathrm{ergs} / \mathrm{cm}^{2} \mathrm{~s}\right)$, the maximum obtainable efficiency is $15 \%$. We shall see later, however, that materials and designs are available that are capable of realizing higher efficiencies with lower power levels.

\subsubsection{Anisotropic Diffraction}

Optical materials such as glass, or crystals with cubic structure, are isotropic with respect to their optical properties; that is, they do not vary with direction. Many crystals, on the other hand, are of such structure, or symmetry, that their optical properties depend on the direction of polarization of the light in relation to the crystal axes. They are birefringent; that is, the refractive index is different for different direction of light polarization.

The theory of diffraction of light thus far presented has assumed that the optical medium is isotropic, or at least that it is not birefringent. A number of important $\mathrm{AO}$ devices make use of the properties of birefringent materials, so a brief description of the important characteristics of anisotropic diffraction will be given here. The essential difference from diffraction in isotropic media is that the momentum of the light,

$$
k=\frac{2 p}{I}=\frac{2 p n}{I_{0}}
$$

will in general, be different for different light polarization directions. Thus, the vector diagram representing conservation of momentum will no longer be the simple isosceles triangle of Figure 11.4a. The momentum vectors for light that is ordinary polarized will terminate on a circle, as shown in Figure 11.6, while those for light that is extraordinary polarized will terminate on the ellipse of Figure 11.6.

To understand the effect of anisotropy on diffraction, it is necessary to mention another phenomenon that occurs when light interacts with shear acoustic waves, that is, waves in which the displacement of matter is perpendicular to the direction of propagation of the acoustic wave. A shear acoustic wave may cause the direction of polarization of the diffracted light to be rotated by $90^{\circ}$. The underlying reason for this is that the shear disturbance induces a birefringence which acts upon the incident light as does a birefringent plate; in other words, it causes the plane of polarization to be rotated. This phenomenon occurs in isotropic materials as well as in anisotropic materials; however, in isotropic materials the momentum vector, $k=2 \pi n / \lambda_{0}$, will be the same for both polarizations, so there is no effect on the diffraction relations. Suppose, instead, that the interaction occurs in a birefringent crystal in a plane containing the optic axis. Let us choose the example as shown in the index surfaces in Figure 11.6, in which the incident light is an extraordinary ray and the diffracted light is an ordinary ray. For this example,

$$
k_{i}=\frac{2 p n_{e}}{I_{0}} \text { and } k_{d}=\frac{2 p n_{0}}{I_{0}}
$$

and the angles of incidence, $\theta_{i}$, and diffraction, $\theta_{d}$, are in general not equal. The theory of anisotropic diffraction was developed by Dixon, ${ }^{5}$ in whose work the expressions for the 


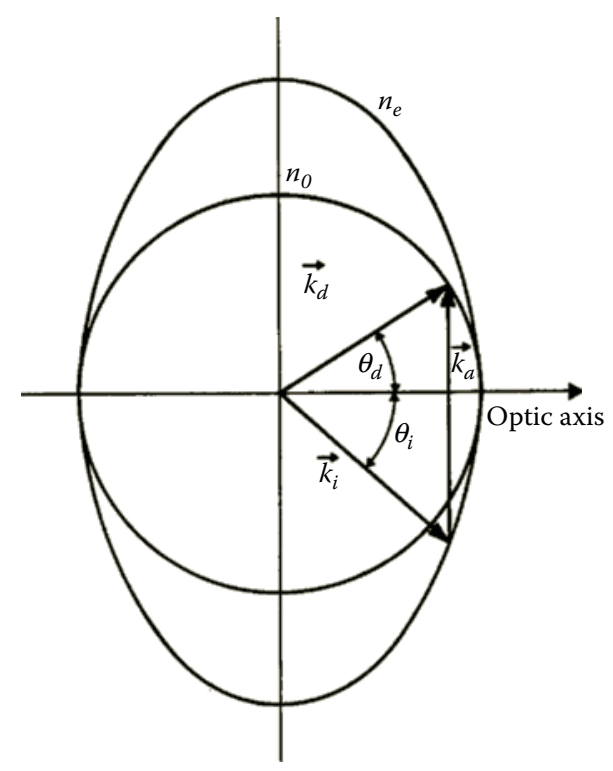

FIGURE 11.6

Vector diagram for diffraction in birefringent medium.

anisotropic Bragg angles were derived as

$$
\begin{aligned}
& \sin q_{i}=\frac{1}{2 n_{i}} \frac{I_{0} f}{n}\left[1+\left(\frac{n}{I_{0} f}\right)^{2}\left(n_{i}^{2}-n_{d}^{2}\right)\right] \\
& \sin q_{d}=\frac{1}{2 n_{d}} \frac{I_{0} f}{n}\left[1-\left(\frac{n}{I_{0} f}\right)^{2}\left(n_{i}^{2}-n_{d}^{2}\right)\right]
\end{aligned}
$$

where $n_{i}$ and $n_{d}$ are the refractive indices corresponding to the incident and the diffracted light polarizations, and $f$ is the acoustic frequency,

$$
f=\frac{n}{\Lambda}
$$

These angles are plotted in Figure 11.7 about that frequency $f_{m}$, for which there is a minimum in the angle of incidence. These curves, the general shapes of which are similar for all birefringent crystals, have a number of interesting characteristics that are useful for several types of $\mathrm{AO}$ devices. The minimum frequency for which an interaction may take place corresponds to $\theta_{i}=90^{\circ}$ and $\theta_{d}=-90^{\circ}$, for which all three vectors will be collinear, as shown in Figure 11.8. It is easily shown that for this case, since the vector equation for conservation of momentum can be written as a scalar equation,

$$
\left|k_{i}\right|+|K|=\left|k_{d}\right|
$$




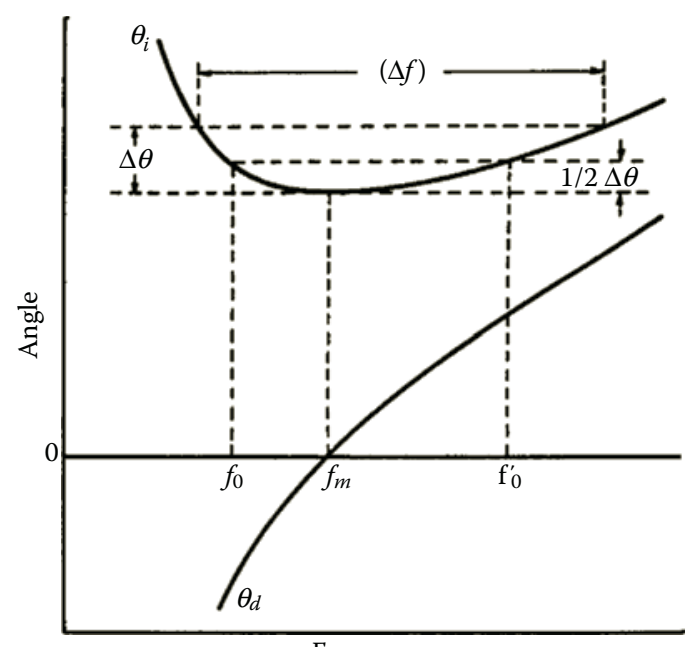

Frequency

\section{FIGURE 11.7}

Angles of incidence and diffraction for anisotropic biréfringent diffraction.

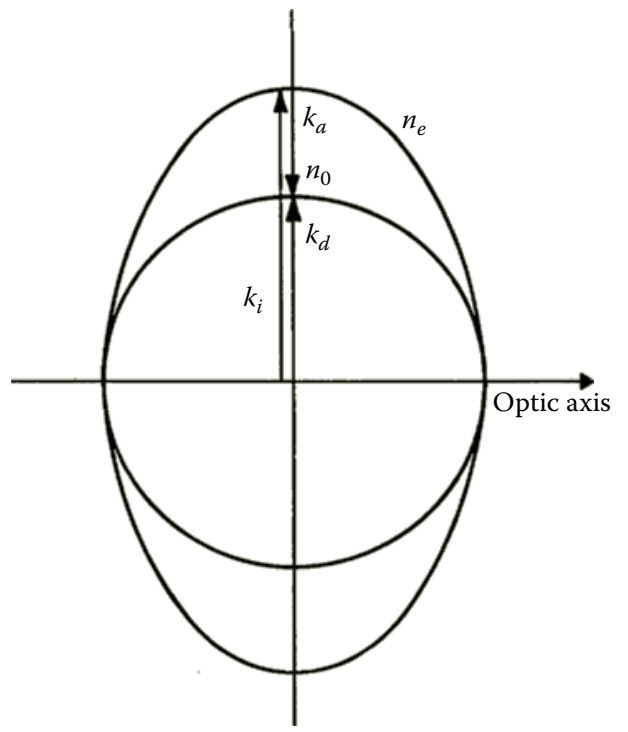

FIGURE 11.8

Vector diagram for collinear diffraction in biréfringent medium.

the frequency for which collinear diffraction takes place is

$$
f=\frac{n\left(n_{i}-n_{d}\right)}{I_{0}}
$$

Such collinear phase matching has been used as the basis of an important device, the electronically tunable $\mathrm{AO}$ filter. ${ }^{6}$ Note that if the incident light had been chosen as ordinary rather than extraordinary polarized, the sense of the acoustic vector $K$ would be reversed. 
In fact, the roles of the two curves in Figure 11.7 would be reversed by interchanging $n_{i}$ and $n_{d}$.

Another interesting region of anisotropic diffraction occurs at the minimum value in the curve representing $\theta_{i}$, at which frequency $\theta_{d}=0$. This frequency, $f_{m}$, is obtained by setting the quantity in brackets in Equation 11.31 equal to zero:

$$
f=\frac{u}{I_{0}} \sqrt{n_{i}^{2}-n_{d}^{2}}
$$

The significance of this point is that the angle of incidence of a scanned beam is relatively insensitive to change over a very broad range of frequencies. This frequency has important implications for the design of scanners because the bandwidth can be much greater than for a comparable isotropic scanner. Because the incident beam angle reaches a minimum value while the diffracted beam angle passes through zero at this point, and increases approximately linearly with frequency, the Bragg angle matching can be maintained over a large range, as will be described later. It will be seen that it is very difficult to achieve an interaction bandwidth this large by any other method.

The description of the interaction of light with sound we have given above is perhaps the simplest in terms of giving an intuitive understanding of the phenomena. Other descriptions, with totally different mathematical formalisms, have been carried out, and these lead to many details and subtleties in the behavior of AO systems that are beyond the scope of this book. Exact calculations have been carried out to extend the range of validity $^{7}$ from the limits allowed by the Raman-Nath theory, ${ }^{8}$ and this has been experimentally investigated. ${ }^{9}$ Other studies have also been carried out to give accurate numerical results for the intensity distribution of light in the various diffraction orders. ${ }^{10}$ The diffraction process has been reviewed and analyzed by Klein and Cook, ${ }^{11}$ using a coupled mode formulation, and there is continuing recent interest in refining the plane-wave scattering theory to give explicit results for intermediate cases. ${ }^{12,13}$ Finally, the AO interaction can be viewed as a parametric process in which the incident optic wave mixes with the acoustic wave to generate polarization waves at sum and difference frequencies, leading to new optical frequencies; this approach has been reviewed by Chang. ${ }^{14}$

One of the most remarkable materials to have appeared recently for AO applications is paratellurite $\left(\mathrm{TeO}_{2}\right) \cdot{ }^{15}$ It has a unique combination of properties, which leads to an extraordinarily high figure of merit for a shear-wave interaction in a convenient RF range. It will be recalled that the anisotropic Bragg relations of Equations 11.30 and 11.31 led to a particular frequency, given by Equation 11.35, for which the angle of incidence is a minimum and therefore satisfies the Bragg condition over a wide frequency range. However, typical values of birefringence place this frequency around $1 \mathrm{GHz}$ or higher. Of particular interest in $\mathrm{TeO}_{2}$ is its optical activity for light propagating along the c-axis, or (001) direction; the indices of refraction for left- and right-hand circularly polarized light are different, so that plane polarized light undergoes a rotation of its plane of polarization by an amount

$$
R=\frac{2 n_{0}}{1} d
$$

where $\delta$ is the index splitting between left- and right-hand polarized light,

$$
d=\frac{n_{1}-n_{r}}{2 n_{0}}
$$


Just as acoustic shear waves can phase-match two linearly polarized light waves, they can also phase-match two oppositely circularly polarized light waves. Thus, shear waves propagating in the (110) direction, with shear polarization in the (110) direction, will diffract left- or right-hand polarized light propagating along the (001) direction, one into the other. The anisotropic Bragg relations apply to crystals with optical activity, where the birefringence is interpreted as

$$
\Delta n=n_{1}-n_{r}=2 n_{0} d
$$

and the value of $\delta$ obtained from specific rotation is wavelength dependent. For the light and sound wave propagation directions described above, the acoustic velocity is $0.62 \times 10^{5}$ $\mathrm{cm} / \mathrm{s}\left(0.24 \times 10^{5} \mathrm{in} / \mathrm{s}\right)$ and the figure of merit, $\mathrm{M}_{2}$, is 515 relative to fused quartz. The frequency for which the Bragg angle of incidence is a minimum, as evaluated from Equation 11.35 for $\lambda=0.633 \mu \mathrm{m}$, is $f=42 \mathrm{MHz}$, a very convenient frequency. For other important wavelengths, the minima occur at $36 \mathrm{MHz}$ for $\lambda=0.85 \mu \mathrm{m}$ and at $22 \mathrm{MHz}$ for $\lambda=1.15 \mu \mathrm{m}$.

The application of the anisotropic Bragg equations to optically active crystals was discussed in detail by Warner et al. ${ }^{16}$ They showed that near the optic axis the indices of refraction are approximated by the relations (for right-handed crystals, $n_{r}<n_{1}$ )

$$
\frac{n_{r}^{2}(q) \cos ^{2} q}{n_{0}^{2}(1-d)^{2}}+\frac{n_{r}^{2}(q) \sin ^{2} q}{n_{1}^{2}}=1
$$

and

$$
\frac{n_{1}^{2}(q) \cos ^{2} q}{n_{0}^{2}(1+d)^{2}}+\frac{n_{1}^{2}(q) \sin ^{2} q}{n_{0}^{2}}=1
$$

For incident angles near zero with respect to the optic axis and for small values of $\delta$,

$$
n_{r}^{2}=n_{0}^{2}\left(1-2 d+\frac{n_{1}^{2}-n_{0}^{2}}{n_{1}^{2}} \sin ^{2} q\right)
$$

and

$$
n_{1}^{2}=n_{0}^{2}\left(1+2 d \cos ^{2} q\right)
$$

For light incident exactly along the optic axis the two refractive indices are simply

$$
n_{r}=n_{0}(1-d)
$$

and

$$
n_{1}=n_{0}(1+d)
$$


The anisotropic Bragg equations for optically active crystals are obtained by substitution of Equations 11.41 and 11.42 into Equation 11.30 and 11.31 for $n_{i}$ and $n_{d}$. By ignoring the higher-order terms, this results in

$$
\sin q_{i} \cong \frac{I f}{2 n_{0} n}\left[1+\frac{4 n_{0}^{2} n^{2}}{I^{2} f^{2}} d+\frac{\sin ^{2} q_{r} n_{0}^{2}}{I^{2} f^{2}}\left(\frac{n_{1}^{2}-n_{0}^{2}}{n_{e}^{2}}\right)\right]
$$

and

$$
\sin q_{d} \cong \frac{I f}{2 n_{0} n}\left[1-\frac{4 n_{0}^{2} n^{2}}{I^{2} f^{2}} d-\frac{\sin ^{2} q_{e} n_{0}^{2}}{I^{2} f^{2}}\left(\frac{n_{1}^{2}-n_{0}^{2}}{n_{0}^{2}}\right)\right]
$$

The anisotropic Bragg angles (as measured external to the crystal) are shown in Figure 11.9 for $\mathrm{TeO}_{2}$ at $\lambda=0.6328 \mu \mathrm{m}$. It is obvious that for frequencies around the minimum, it will be possible to achieve a much larger bandwidth for a given interaction length than is possible with normal Bragg diffraction; a one-octave bandwidth corresponds to a variation in angle of incidence for perfect phase matching of only $0.16^{\circ}$. A useful advantage of such operation is that large bandwidths are compatible with large interaction lengths, which avoids higher diffraction orders from Raman-Nath effects. For normal Bragg diffraction, on the other hand, large bandwidths can only be reached with interaction lengths that are so small that significant higher-order diffraction occurs. This decreases the efficiency with which light can be directed to the desired first order, and also limits the bandwidth to less than one octave in order to avoid overlapping low-frequency second-order with higher-frequency first-order diffracted light. However, tellurium dioxide operating in the

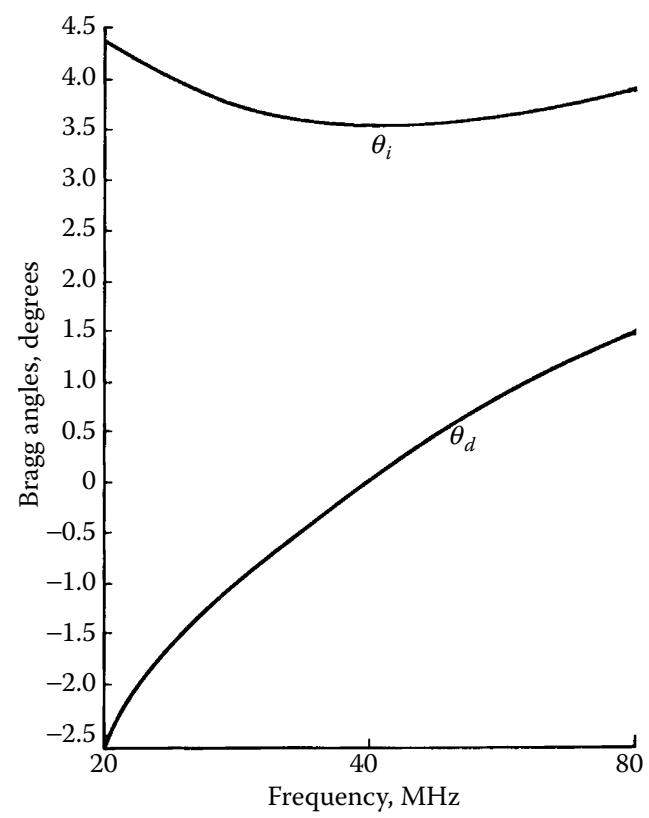

FIGURE 11.9

Bragg angles of incidence and diffraction (external) for anisotropic $\mathrm{TeO}_{2}$ scanner, $\lambda=0.6328 \mu \mathrm{m}$. 
anisotropic mode can always be made to diffract in the Bragg mode with no bandwidth limitation on length, so that this feature combined with the extraordinary high figure of merit leads to deflector operation with very low drive powers.

An important degeneracy occurs for anisotropic Bragg diffraction, which causes a pronounced dip in the diffracted light intensity at the midband frequency, where $\theta_{i}$ has its minimum. This degeneracy was explained by Warner et al., ${ }^{16}$ and is easily understood by referring to the diagram in Figure 11.10. Two sets of curves are shown in the figure; the solid pair represents $\theta_{i}$ and $\theta_{d}$ when the incident light momentum vector has a positive component along the acoustic momentum vector, and the dotted pair represents these angles when the incident light momentum vector has a negative component along the acoustic vector. In the former case, the frequency of the diffracted light is upshifted, and in the latter it is downshifted. The vector diagram for this process is shown in Figure 11.11. Light is incident to the acoustic wave of frequency $f_{0}$ at an angle $\theta_{0}$, and is diffracted as a frequency upshifted beam, $\left(v+f_{0}\right)$, normal to the acoustic wave. This light, in turn, may be rediffracted; referring to Figure 11,.10, it can be seen that for a frequency $f_{0}$ light that is incident at $\theta=0^{\circ}$ can be rediffracted to either $\theta_{0}$ or $-\theta_{0}$, In the former case, the light will be downshifted to the original incident light frequency $v$, and in the latter case it is upshifted to $\nu+2 f_{0}$. Note that this degeneracy can only occur at the frequency $f_{0}$ where light incident normal to the acoustic wave is phase matched for diffraction into both $\theta_{0}$ and $-\theta_{0}$. How the

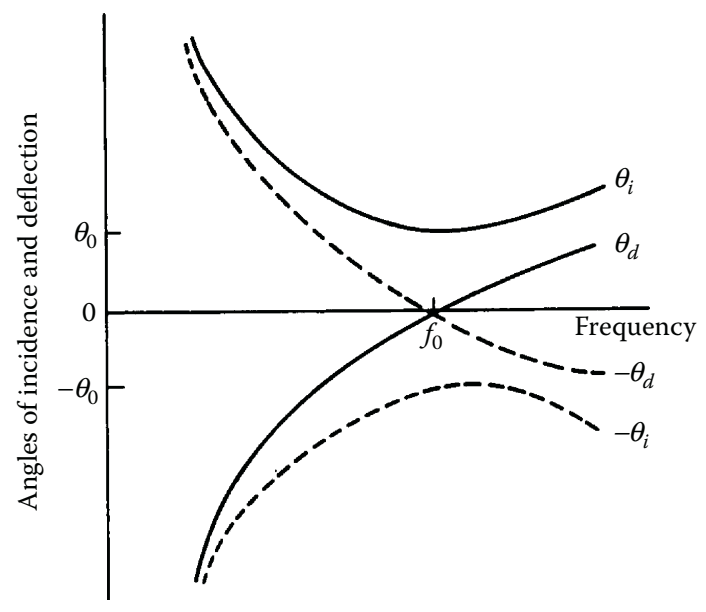

\section{FIGURE 11.10}

Angles of incidence and diffraction for anisotropic diffraction. Solid curves are for incident light having a component in the same direction as the acoustic wave, and dotted curves are for incident light having a component in the opposite direction.

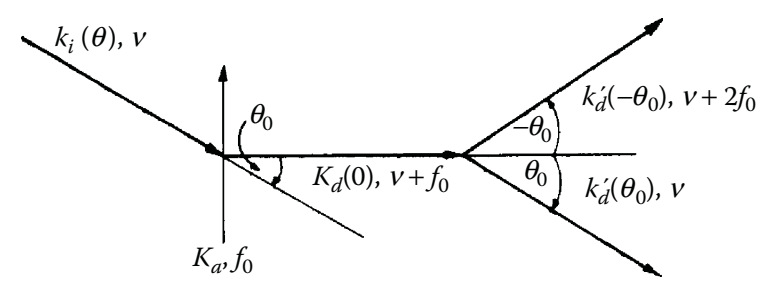

FIGURE 11.11

Vector diagram for midband degeneracy of Bragg diffraction in birefringent medium. 


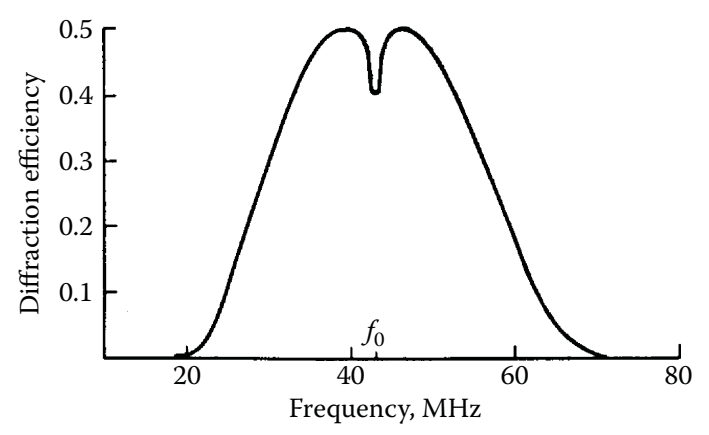

\section{FIGURE 11.12}

Effect of midband degeneracy on diffraction efficiency, for a maximum efficiency of $50 \%$.

light is distributed in intensity between the three modes will depend upon the interaction length and the acoustic power level. The exact solution to this is found by setting up the coupled mode propagation equations under phase-matched conditions. The result of this is that maximum efficiency for deflection into the desired mode at $f_{0}$ is $50 \%$. At low acoustic power, the deflection of light into the undesired mode is negligible; at high powers the unwanted deflection increases so that, for example, if the efficiency is $50 \%$ for frequencies away from $f_{0}$, it will be $40 \%$ at $f_{0}$. The theoretical response of such a deflector is shown in Figure 11.12, and is in excellent agreement with experimental results.

\subsection{ACOUSTO-OPTIC MODULATOR AND DEFLECTOR DESIGN}

\subsubsection{Resolution and Bandwidth Considerations}

Resolution, bandwidth, and speed are the important characteristics of AO scanners, shared by all types of scanning devices. Depending upon the application, only one, or all of these characteristics may have to be optimized; in this section, we will examine which AO design parameters are involved in the determination of resolution, bandwidth, and speed. Consider an AO scanner with a collimated incident beam of width $D$, diffracted to an angle $\theta_{0}$ at the center of its bandwidth $\Delta f$. If the diffracted beam is focused onto a plane by a lens, or lens combination, at the scanner, the diffraction spread of the optical beam will be

$$
d x=F d f \cong \frac{F I}{D}
$$

where $F$ is the focal length of the lens. The light intensity will be distributed in the focal plane as illustrated in Figure 11.13. As an example for diffraction-limited optics, the spot size for a $25 \mathrm{~mm}$ ( $1 \mathrm{in}$ ) wide light beam of wavelength $6.33 \mu \mathrm{m}$ at a distance of $30 \mathrm{~cm}$ (12 in) from the delay line is $7.6 \mu \mathrm{m}\left(3 \times 10^{-4} \mathrm{in}\right)$. There are, however, aberrations that prevent this from being fully realized, as will be discussed later.

The number of resolvable spots will be the angular scan range divided by the angular diffraction spread,

$$
N=\frac{\Delta q}{d f}
$$




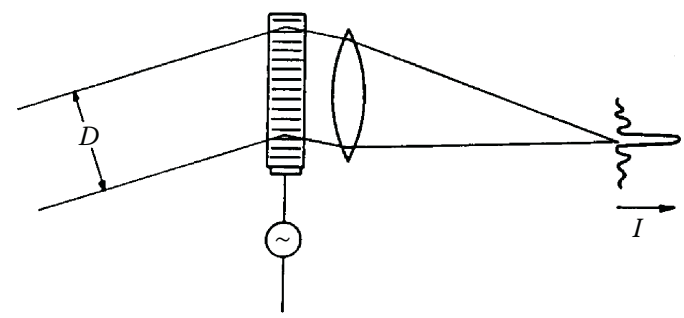

FIGURE 11.13

Distribution of light intensity due to diffraction by acoustic field.

where $\Delta \theta$ is the range of the angular scan. Differentiating the Bragg angle formula yields

$$
\Delta q=\frac{1}{n \cos q_{0}} \Delta f
$$

and

$$
N=(\Delta f)\left(\frac{D}{n \cos q_{0}}\right)=\Delta f t
$$

where $\tau$ is the time that it takes the acoustic wave to cross the optical aperture. The resulting expression is the time-bandwidth product of the AO scanner, a concept applied to a variety of electronic devices as a measure of information capacity. The time-bandwidth product of an AO Bragg cell is equivalent to the number of bits of information that may be instantaneously processed by the system. For an acousto-optic modulator (AOM) that is strictly a temporal modulator, a time-bandwidth product near unity is generally desired as the goal is often to modulate as fast as possible and therefore to minimize the aperture delay time. In contrast, for an AOD the time-bandwidth product is generally desired to be as large as possible to produce a large number of resolution elements.

There are two factors limiting the bandwidth of an AO device: the bandwidth of the transducer structure and the acoustic absorption in the delay medium. The acoustic absorption increases with increasing frequency; for high-purity single crystals the increase generally goes with the square of the frequency. For glassy materials, on the other hand, the attenuation will increase more slowly with frequency, often approaching a linear function. The maximum frequency is generally taken as that for which the attenuation of the acoustic wave across the optical aperture is equal to $3 \mathrm{~dB}$. A reasonable approximation of the maximum attainable bandwidth is $\Delta f=0.7 f_{\max }$, so that we may derive some relationships for the maximum number of resolution elements.

For a material with a quadratic dependence of attenuation on frequency,

$$
a(f)=\Gamma f^{2}
$$

and the maximum aperture for $3 \mathrm{~dB}$ loss is

$$
D=\frac{3}{\Gamma f^{2}}
$$


Using these results, the maximum number of resolution elements is

$$
N_{\max } \simeq q \sqrt{\frac{1.5 D}{u^{2} \Gamma}}
$$

from which it can be seen that, in principle, it is always advantageous to make the delay line as long as possible. In practice, the aperture will be limited by the largest crystals that can be prepared, or ultimately by the size of the optical system. For a glassy material for which the attenuation increases linearly with frequency,

$$
a(f)=\Gamma^{\prime} f
$$

and the maximum number of resolvable spots will be

$$
N_{\max } \simeq \frac{2}{\Gamma^{\prime} u}
$$

which is independent of the size of the aperture, being determined only by the material attenuation constant and the acoustic velocity.

In the next section we will review material considerations in some detail, and see what the performance limits are of currently available AO materials. As a numerical example, however, the highest-quality fused quartz has an attenuation of about $3 \mathrm{~dB} / \mathrm{cm}$ at $500 \mathrm{MHz}$ and an acoustic velocity of $5.96 \times 10^{5} \mathrm{~cm} / \mathrm{s}\left(2.35 \times 10^{5} \mathrm{in} / \mathrm{s}\right)$ (for longitudinal waves), leading to $N_{\max }=560$.

\subsubsection{Interaction Bandwidth}

The number of resolution elements will be determined by the frequency bandwidth of the transducer and delay line, but a number of other bandwidth considerations are also of importance for the operation of a scanning system. While a large value of $\tau$ leads to a large value of $N$, the speed of the device is just equal to $l / \tau$. That is, the position of a spot cannot be changed randomly in a time less than $\tau$. If the acoustic cell is being used to temporally modulate the light as well as to scan, then obviously the modulation bandwidth will similarly be limited by the travel time of the acoustic wave across the optical aperture. In order to increase the modulation bandwidth, the light beam must be focused to a small width, $\omega$, in the acoustic field. The $3 \mathrm{~dB}$ modulation bandwidth is approximately

$$
\Delta f=\frac{0.75}{t}=\frac{0.75 u}{w}
$$

and the diffraction-limited beam waist (the $1 / e^{2}$ power points) of a Gaussian beam is

$$
w_{0}=\frac{2 I_{0} F}{p D}
$$

where $D$ is the incident beam diameter and $F$ is the focal length of the lens. With this value of beam waist, the maximum modulation bandwidth is

$$
\Delta f=0.36 p \frac{u D}{I_{0} F}
$$


It can be seen from Equation 11.58 that the modulation bandwidth for a diffraction-limited focused Gaussian beam can be very high; for example, for a material of acoustic velocity $5 \times 10^{5} \mathrm{~cm} / \mathrm{s}\left(2 \times 10^{5} \mathrm{in} / \mathrm{s}\right)$ the bandwidth of a $0.633 \mu \mathrm{m}$ light beam focused with an $\mathrm{f} / 10$ lens is about $1 \mathrm{GHz}$. Such a system, however, is practically useless, because the diffraction efficiency would be extremely small.

In order for the Bragg interaction bandwidth to be large, there must be a large spread of either the acoustic or the optical beam directions, $\delta \theta_{a}$ and $\delta \theta_{0}$, respectively, or both. This spread may occur either by focusing, which in the case of the acoustic beam is achieved by curving the plane of the transducer, or it may be due simply to the aperture diffraction for both beams. It follows from fairly simple arguments that the optimum configuration for the most efficient utilization of optical and acoustic energy corresponds to approximately equal angular spreading, $\delta \theta_{0} \cong \delta \theta_{a}$, as illustrated in Figure 11.14.

For an AOD, the angular spread of the acoustic beam should be made large enough to match Bragg diffraction over the frequency range of the transducer-driving circuit bandwidth. As mentioned previously, this will result in some reduction in efficiency. To examine the relationship between bandwidth and the efficiency, we must first state another wellknown result of acoustically diffracted light. This is, as shown by Cohen and Gordon, ${ }^{17}$ that the angular distribution of the diffracted light will represent the Fourier transform of the spatial distribution of the acoustic beam. This Fourier transform pair is illustrated in Figure 11.15 for the usual case of the rectangular acoustic beam profile. It seems intuitively obvious for this simple case, in which the diffraction spread of the incident optical beam is ignored, that there will be components in the diffracted light corresponding to the acoustic field side lobes. It is shown in Reference 17 that the Fourier transform relationship holds for an arbitrary acoustic beam profile.

For the rectangular profile, the angular dependence of the diffracted light, illustrated in Figure 11.15, is

$$
\frac{I(q)}{I_{0}} \propto\left[\frac{\sin \frac{1}{2} K L\left(q-q_{B}\right)}{\frac{1}{2} K L\left(q-q_{B}\right)}\right]^{2}
$$

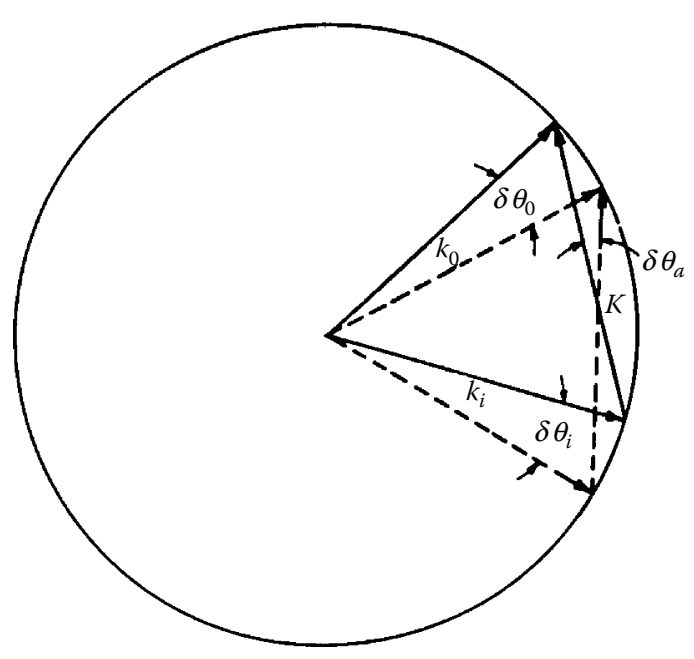

FIGURE 11.14

Vector diagram for Bragg diffraction in isotropic medium with angular spread of acoustic beam direction. 


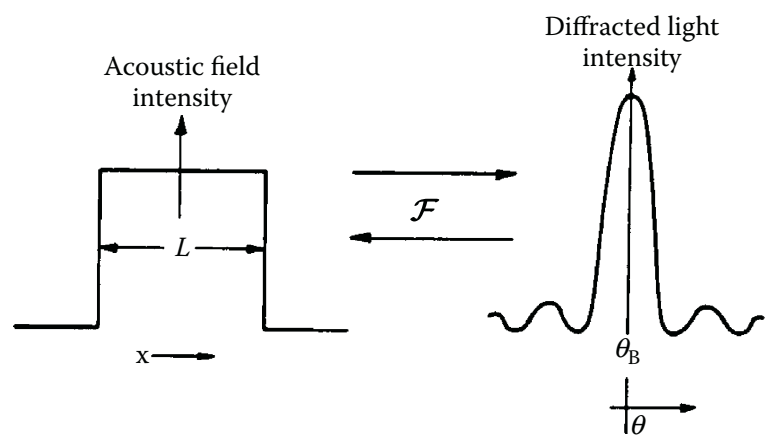

FIGURE 11.15

Fourier transform relationship between acoustic field intensity and diffracted light intensity.

for which the $-3 \mathrm{~dB}$ points occur at

$$
\frac{1}{2} K L(\Delta q)_{1 / 2} \quad \pm 0.45 p
$$

where $(\Delta \theta)_{1 / 2}$ is the value of $\theta-\theta_{\mathrm{B}}$ at the half-power points. This yields a value for the angular width of the optical beam, just equal to the diffraction spread of the acoustic beam, namely

$$
2(q)_{1 / 2} \frac{1.8 p}{K L}
$$

The frequency bandwidth is obtained by equating this result to the differential of the Bragg condition:

$$
d q=\frac{l_{0} \Delta f}{n u \cos q_{B}}
$$

This result is

$$
\Delta f=\frac{1.8 n u^{2} \cos q_{B}}{L f_{0} I_{0}}
$$

For AO scanning devices in which the bandwidth as well as the diffraction efficiency is of importance, a more relevant figure of merit may be the product of the bandwidth with the efficiency. By combining Equations 11.63 and 11.25, this product is

$$
2 f_{0} \Delta f \cdot \frac{I_{1}}{I_{0}}=\frac{1.8 p^{2}}{I_{0}^{3} H \cos q_{B}}\left[\frac{n^{7} p^{2}}{r u}\right] P
$$


The quantity in brackets can be regarded as the figure of merit of the material when the efficiency-bandwidth product is the important criterion, and is designated as

$$
M_{1}=\frac{n^{7} p^{2}}{r u}
$$

Other methods of achieving a large interaction bandwidth include transducer designs that steer the acoustic beam in direction in order to track the Bragg angle as it changes with frequency. A description of beam steering will be included in the section on transducers. Still another figure of merit was introduced by Dixon ${ }^{18}$ in connection with wideband AO devices. Because the power requirements decrease as the transducer height $H$ decreases, it is advantageous to make $H$ as small as possible. If there are no limitations on the minimum size of $H$, it can be as small as the optical beam waist in the region of the interaction, $h_{\min }$. The modulation bandwidth is determined by the travel time of the acoustic wave across this beam waist,

$$
t \approx \frac{1}{\Delta f}=\frac{h_{\min }}{n}
$$

so that

$$
h_{\min }=\frac{n}{\Delta f}
$$

Substitution of this value for $H$ in Equation 11.62 results in the relation

$$
2 f_{0} \frac{I_{1}}{I_{0}}=\frac{1.8 p^{2}}{I_{0}^{3} \cos q_{B}}\left[\frac{n^{7} p^{2}}{r u^{2}}\right]
$$

and the appropriate figure of merit for this situation is the quantity in brackets:

$$
M_{3}=\left[\frac{n^{7} p^{2}}{r u^{2}}\right]
$$

Note that the optical wavelength appears as $I_{0}^{3}$ in both Equations 11.64 and 11.68 , so that operation at long wavelengths is relatively more difficult in terms of power requirements for configurations optimizing bandwidth as well as efficiency.

\subsubsection{Deflector Design Procedure}

The useful optical aperture of a Bragg cell is usually considered to be that length across which the difference in acoustic attenuation between the highest and the lowest frequencies within the operating bandwidth of the cell is $3 \mathrm{~dB}$. A particular application may dictate either a bandwidth or a resolution, that is, a time-bandwidth product. In general, an optimized Bragg cell design will maximize the number of resolvable spots, as well as other transducer structure parameters. 
The number of resolvable spots, or the time-bandwidth product, will be determined by three key factors: ${ }^{19}$ the acoustic attenuation $\Gamma$, the optical aperture of the AO crystal $D$, the angular beam spreading of the acoustic wave, which is determined by the transducer length $L$, and the acoustic wavelength. The constraints placed upon the number of resolvable spots $N$ by these three factors is given by the relations ${ }^{19}$

$$
\begin{aligned}
& N \leq \frac{1.5 \Lambda_{c}}{\Gamma \Lambda_{1}^{2}} \\
& N \leq \frac{D}{2 \Lambda_{c}} \\
& N \leq\left(\frac{L}{2 \Lambda_{c}}\right)^{2}
\end{aligned}
$$

where $\Lambda_{c}$ is the acoustic wavelength at the center frequency, $\Lambda_{1}$ is the acoustic wavelength at $1 \mathrm{GHz}$, and $\Gamma$ is the acoustic attenuation in $\mathrm{dB}$ per unit length, normalized to $1 \mathrm{GHz}$ (under the usual assumption that the attenuation increases quadratically with frequency). Note that Equation 11.70 allows for a 3-dB attenuation. Once the center frequency and the bandwidth of the cell have been determined, the transducer structure must be designed. This will include the electrode length $L$ and height $H$. The length must be chosen so that it is small enough to allow sufficient beam spread to satisfy the Bragg angle matching requirements over the desired bandwidth (for a fixed angle of incidence of the optical beam). At the same time, the diffraction efficiency will decrease as $L$ decreases, so that we will want $L$ to be as large as possible within the interaction bandwidth constraint.

\subsubsection{Modulator Design Procedure}

$\mathrm{AOD}$ and $\mathrm{AOM}$ have very similar design requirements and in some cases, one design may be suitable for either application or both. While the key design parameter for deflectors is typically the number of resolvable spots, the key design parameter for modulators is typically rise time or modulation bandwidth. These differing design parameters lead to the characteristic that, for deflectors, the optical aperture is typically made as large as possible, while for modulators, the optical beam is made as small as possible.

The rise time of an AOM is fundamentally limited by the acoustic velocity of the modulator material. When an acoustic pulse is transmitted from the transducer, diffraction will begin when the leading edge of the pulse reaches the optical beam. Full, diffracted beam power will not be obtained until the acoustic wavefront reaches the opposite end of the optical beam. The shape of the rising optical pulse will depend on the shape of the optical beam.

For a Gaussian optical beam, the time required for the acoustic wave to cross the $1 / e^{2}$ beam diameter is

$$
t=\frac{D_{1 / e}^{2}}{V_{\mathrm{a}}}
$$

where $V_{\mathrm{a}}$ is the acoustic velocity. This beam width corresponds to a rise time from $2.3 \%$ to $97.7 \%$. The more conventional rise time from $10 \%$ to $90 \%$ is calculated as

$$
t_{R}=0.64 t
$$


For video modulation applications, the rise time limits the frequency response of the modulator. The modulator bandwidth can be expressed as the frequency at which $3 \mathrm{~dB}$ roll-off occurs, which is estimated by the standard relation

$$
f_{0}=\frac{0.35}{t_{R}}
$$

In the case of square pulse video modulation, the modulation speed may be defined by a specific dynamic extinction ratio requirement. For square wave modulation at frequency $f_{0}$, the dynamic extinction ratio is approximately 10:1. For high extinction ratios on the order of 1000:1, the maximum square wave modulation frequency is approximately $f_{0} / 2$. For a given rise time, the design beam diameter can be calculated using the above relations. Note that the optical beam cannot be made arbitrarily narrow because the beam must remain relatively collimated over the acoustic interaction length $L$. If the beam waist is too small, the beam divergence over the length $L$ will result in a longer rise time than predicted based on the beam waist. The minimum value of $L$ is constrained by the need to stay in the Bragg regime (Sec. 2.2) and achieve a specified diffraction efficiency (Sec. 2.3).

The above discussion of dynamic extinction ratio assumes that the static extinction ratio is not limiting. The limit of static extinction ratio is determined by the ability to discriminate the diffracted beam from undiffracted or scattered light. Scattered light is a function of the quality of the material and the surface finish of the AO cell and is typically the limiting parameter for static extinction ratio when the diffracted beam separation is made large enough. The beam separation between the zero- and first-order beams is given by

$$
\Delta f=\frac{I}{\Lambda}
$$

If the separation angle is made equal to the full divergence angle of the optical beam and a knife edge placed halfway between the zero- and first-order beams, approximately $2.3 \%$ of the blocked beam will pass the knife edge. This means the minimum static extinction ratio would be about 40:1. If the beam separation is increased to twice the beam angle, the optical power passing the knife edge is decreased to $0.003 \%$. For most applications, this amount of beam separation is sufficient to make the extinction ratio limitation due to beam separation negligible. Using this condition with the formula of divergence of a Gaussian beam gives

$$
\Delta f>\frac{81}{p D_{0}}
$$

Combining the above two equations gives the following condition for beam separation

$$
\Lambda>\frac{p D_{0}}{8}
$$

Another consideration is that the angular acceptance window of the acoustic field should be large enough to allow Bragg interaction over the optical beam. If the angular acoustic field is too narrow, the optical beam will be apodized in angle, resulting in output beam 
distortion and decreased diffraction efficiency. The angular acoustic intensity from a rectangular transducer is described by

$$
I(q)=\sin c^{2}\left[\frac{q}{(\Lambda / L)}\right]
$$

The null-to-null width is therefore $2 \pi \Lambda / L$. This width should be much greater than the $1 / e^{2}$ beam angle to maintain good diffraction efficiency and prevent distortion. Using the formula for beam divergence, the condition for $L$ becomes

$$
L \ll \frac{p^{2} \Lambda D_{0} n}{21}
$$

Remember that for Bragg interaction $L$ cannot be made arbitrarily small. In practice, the length is chosen to be as short as tolerable based on requirements for efficiency and suppression of higher orders, typically such that Raman-Nath parameter $Q \approx 12$.

\subsection{SPECIALIZED ACOUSTO-OPTIC DEVICES FOR SCANNING}

\subsubsection{Acoustic Traveling Wave Lens}

Most AO applications are based on diffraction effects, requiring interaction over at least a few periods sinusoidal index change in the acoustic media. However, it is also possible to use the index change over a fraction of a period to act as a lens to focus light by refraction. In this case, the index change produced by a segment of an acoustic wave can be viewed as a gradient-index cylinder lens moving with the speed and direction of the acoustic wave.

Consider a conventional scanning system with a single axis scan device, in this case an AO Bragg deflector, followed by a scan lens. The total number of resolvable spots in the scan is determined by the aperture size and scan angle of deflector, and can be determined approximately as the product of the bandwidth and the acoustic transit time (Equation 11.50). The scan lens can be altered to change the scan length and spot size, but the total number of spots remains the same. However, the number of spots may be increased by adding a traveling lens after the scan lens as shown in Figure 11.16. In this case, the speed and timing of the deflector is synchronized with the speed and phase of the acoustic traveling wave such that the input beam tracks the acoustic lens as it propagates in the scan direction. The acoustic lens reduces the scanned spot size but has no effect on the scan length, thereby producing spot gain.

\subsubsection{Design Considerations}

The sinusoidal index variation is approximately parabolic near the index minimum and acts as a focusing (positive) lens. A quarter-wave aperture centered on the index minimum will provide the lens with near diffraction-limited performance. The focal length of the traveling lens was derived by Foster: ${ }^{20}$

$$
F=\left(\frac{\Lambda}{4}\right)\left(\frac{n_{0}}{\Delta n}\right)^{1 / 2}
$$




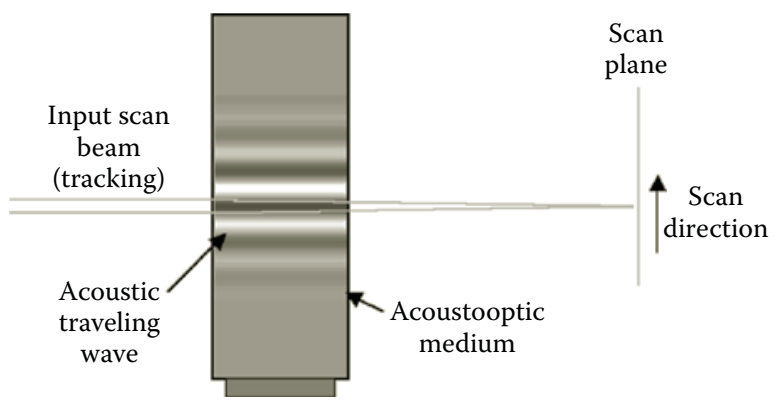

FIGURE 11.16

Application of an acoustic traveling-wave lens.

where $\Lambda$ is the acoustic wavelength, $n_{0}$ is the index of refraction, and $\Delta n$ is the peak refractive index variation. The focal length $(F)$ in Equation 11.81 refers to the focal distance internal to the lens. It can also be viewed as the lens thickness required to produce a quarter-pitch lens. If the thickness of the lens is less than $F$, the effective focal length will be longer.

Foster also derived an expression for the $f$-number of the lens by considering the path of the extreme rays at $\pm \Lambda / 8$ :

$$
f \text {-number }=\frac{F}{D}=\frac{2}{p\left(n_{0} / \Delta n\right)^{1 / 2}}
$$

Assuming a Gaussian beam of diameter $D_{0}\left(1 / e^{2}\right)$ is input to the traveling lens, the size of the focused spot is estimated as

$$
D_{1}=\frac{4 I F}{p n_{0} D_{0}}
$$

Combining Equations 11.81, 11.82, and 11.83 gives an expression for the output spot size from the traveling lens:

$$
D_{1}=\frac{81}{p^{2}\left(n_{0} / \Delta n\right)^{1 / 2}}
$$

The above derivation uses the assumptions that the lens thickness is approximately equal to $F$ and the input beam diameter $\left(D_{0}\right)$ is equal to $\Lambda / 4$. In an application where the traveling lens is the final scan lens, $F$ is typically made less than the lens thickness such that the focus occurs outside the lens and with a back focal distance sufficient to reach the scan plane.

As an example, assume that a prescanner produces a linear scan of $50 \mathrm{~mm}$ with a $0.5-\mathrm{mm}$ diameter beam of wavelength $633 \mathrm{~nm}$. A traveling acoustic lens device made from dense flint glass (SF-59) will be added to provide a final spot size of $0.05 \mathrm{~mm}$ or spot gain of 10. The required refractive index change $(\Delta n)$ is determined to be 0.000157 from Equation 11.84. The acoustic wavelength will be $4 D_{0}$ or $2 \mathrm{~mm}$, and therefore $F$ is determined to be $55.7 \mathrm{~mm}$ from Equation 11.81. 
The length of the acoustic transducer $(L)$, corresponding to the thickness of the lens, is chosen to be $45 \mathrm{~mm}$, which is slightly less than $F$ such that the focus can be outside the traveling lens device. The height of the transducer $(H)$ is chosen to be $15 \mathrm{~mm}$, such that the acoustic near field is longer than the scan length to prevent excessive acoustic loss along the scan direction due to diffraction spreading.

The amount of refractive index change is proportional to the square root of the acoustic intensity:

$$
\Delta n=\left(\frac{M_{2} P_{A}}{2}\right)^{1 / 2}
$$

where $M_{2}$ is the AO figure of merit and $P_{\mathrm{A}}$ is the acoustic intensity. Using Equation 11.85, the acoustic intensity required is $2.6 \mathrm{~W}$ per $\mathrm{mm}^{2}$. The instantaneous power required is therefore $1800 \mathrm{~W}$.

While the power requirement is very large compared to those for typical Bragg cells, the average power required can be reduced significantly by pulsing the acoustic signal once per scan line. Even so, the large instantaneous and average power requirements for traveling acoustic wave devices are a significant challenge to practical implementation. One way to reduce the power requirement is to narrow the AO cell height to a fraction of an acoustic wavelength to form an acoustic slab waveguide. The waveguide properties eliminate the acoustic diffraction spreading problem and associated need for a tall transducer.

\subsubsection{Chirp Lens}

In a typical AOD application, the transducer frequency is swept linearly over a range $\Delta f$ to provide a linear angular scan $(\Delta \theta)$ of the output beam. This frequency sweep is referred to as a chirp. The equivalent length of the acoustic chirp is equal to the product of the chirp time and the acoustic velocity. If the aperture were large enough to cover the entire acoustic chirp, then the diffracted angle would vary over the range $\Delta \theta$ along the chirp length as shown in Figure 11.17. Using the small angle approximation, the acoustic chirp acts as a lens with $f$-number inversely proportional to $\Delta \theta$ :

$$
f \text {-number } \approx \frac{1}{\Delta q} \text { Acoustic apertute } \geq \text { Chirplength }
$$

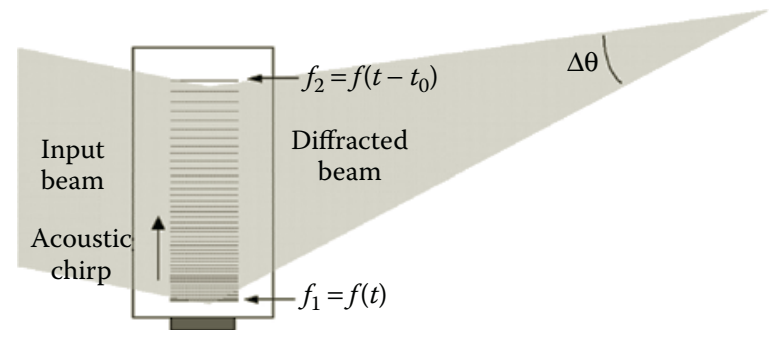

FIGURE 11.17

Focusing effect of an acoustic chirp. 
If the aperture is smaller than the chirp length, as is typically the case, then the $f$-number will be inversely proportional to the fraction of the chirp length covered by the aperture:

$$
f \text {-number } \approx \frac{1}{\Delta q} \frac{T_{\text {chirp }}}{\mathrm{T}} \text { Acoustic apertute }<\text { Chirplength }
$$

For a typical beam deflector application, the chirp length $T_{\text {chirp }}$ is much larger than the acoustic aperture time $T$. In this case, the focusing power of the chirp will be much less than that of an $f-\theta$ lens, and can be neglected. For very fast scanners, where $T_{\text {chirp }}$ approaches $T$, the chirp focusing effect may contribute to the number of scan spots. Note that if $T_{\text {chirp }}=T$, the chirp focusing effect is equivalent to the power of an $f-\theta$ lens. However, this arrangement does not make a useful scanner, as the scan time is approximately the same as the access time.

By making the chirp smaller than the aperture length, the chirp can be used as a traveling lens. The chief application of a traveling chirp lens is to employ it as a post scan lens as described in the previous section. The traveling chirp lens is diffractive, and does not require the high instantaneous acoustic power needed to produce the refractive quarterwave lens with comparable $f$-number. It also allows more flexibility with lens aperture, as the lens size is a function of chirp time and not a function of acoustic frequency. Unlike the refractive lens, the chirp lens is subject to diffractive losses and the diffraction efficiency may not be uniform across the aperture. Another disadvantage is that the quality of the lens is a function of the linearity of the chirp signal, such that phase error in the chirp signal will translate into aberrations in the lens.

\subsubsection{Multichannel Acousto-Optic Modulator}

AOMs are often used in conjunction with a scan beam to produce a raster scan. The scan line rate may be limited by the response time of the AOM, the exposure time, or the scan rate of the scan generating components. One way to increase throughput for a raster scan is to use multiple beams in a parallel scan arrangement. The beams may pass through a common set of scan elements, such that the final scans are identical except for position offset at the scan plane. Although scanned together, each beam must have its own modulation sequence. This can be accomplished by using a multichannel AOM.

Multichannel AOM can be fabricated using the same number of steps as single-channel modulators, with the main difference being that an array of electrodes is deposited on the transducer substrate instead of a single electrode. In application, a parallel array of beams is registered to the transducer array of the modulator as shown in Figure 11.18.

The effect of one channel upon another is of particular concern for multichannel devices, especially as larger numbers of channels are integrated in a single device. Cross talk between channels can occur through a number of mechanisms including electrical cross talk in the feed circuitry or electrodes and acoustic cross talk between adjacent channels. Another cross-talk mechanism is thermal, where the heat load added from turning on one or more channels causes a thermo-optic or strain-optic effect that alters the output of an independent channel. Electrical cross talk is controlled by using good RF design practices including use of controlled impedance microstrip lines and providing good ground continuity from the feed circuitry to the transducer. Acoustic cross talk is dependent on the location of the optical aperture and the spacing between electrodes. Because the acoustic field in isotropic materials will spread once it propagates beyond the near-field zone 


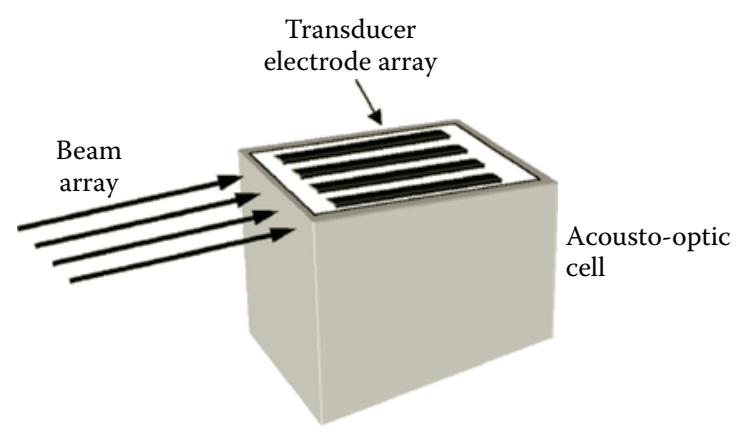

FIGURE 11.18

Multiple transducer channels on a monolithic acousto-optic device.

adjacent to the transducer, the amount of acoustic overlap is generally greater if the optical aperture is placed further from the transducer.

The degree of acoustic overlap between adjacent channels can also be manipulated through the electrode design. For an electrode with a simple rectangular shape, the acoustic intensity will have a sinc ${ }^{2}$ angular distribution, which has side lobes $13 \mathrm{~dB}$ down from the main lobe. Other shapes, such as diamond or Gaussian envelope, can produce much lower side lobe values, although sometimes at the expense of faster spread of the main lobe.

\subsection{MATERIALS FOR ACOUSTO-OPTIC DEVICES}

\subsubsection{General Considerations}

We have seen in the preceding section that two important criteria for choosing materials for $\mathrm{AO}$ scanning systems are the $\mathrm{AO}$ figure of merit and the high-frequency acoustic loss characteristics. Other properties that determine the usefulness of a material are its optical transmission range, optical quality, availability in suitable sizes, mechanical and handling characteristics as they may pertain to polishing and fabrication procedures, and chemical stability under normal conditions. As with most components, cost will be an important factor, even when all the other factors may be positive, if competing techniques are available.

One of the limitations on the use of AO scanners before the late 1960s was the availability of materials with reasonably high figure of merit. As we have seen, fused quartz, which is used as the standard for comparison, has a figure of merit so low that only a few percent diffraction efficiency can be obtained for scanners of typical dimensions, and with RF powers that can be applied without causing damage to transducer structures. Water is a fairly efficient material, with a figure of merit about 100 times larger than fused quartz and has actually found use in some scanning systems. As with most liquids, it cannot be used at frequencies higher than about $50 \mathrm{MHz}$, so that large numbers of resolution elements cannot be achieved. Since the late 1960s, many new materials have been synthesized and existing ones were found to have excellent properties. Materials can now be found for most scanning applications from the UV through the intermediate IR where high bandwidth is required. 
The selection of a material for any particular device will be dictated by the type of operation under consideration. In general, it is desirable to select a material with low-drivepower requirements, suggesting those with large refractive index and low density and acoustic velocity. If, however, high-speed modulation is of paramount importance, then a low acoustic velocity may lead to slower than required speeds. In the following section, we will review the factors and trade-offs involved in the selection of materials for various AO applications. Whatever the particular material requirements may be, there are also a number of practical considerations that dictate several generally important material properties whatever the application: (1) the optical quality must be high so that not only absorption but scattering and large-scale inhomogeneities are small; (2) good chemical stability is required so that protective enclosures are not needed to maintain integrity; (3) good mechanical properties are required so that the device can be cut and polished without extraordinary procedures and can be adjusted and used with normal handling techniques; (4) the availability of crystal growth methods for obtaining suitably large, high-quality boules with reasonable cost is needed; and (5) a low-temperature coefficient of velocity is required to avoid drift of scan properties.

\subsubsection{Theoretical Guidelines}

There is no simple microscopic theory of the photoelastic effect in crystals. Therefore it is not possible to predict the magnitude of the photoelastic constants from first principles. However, Pinnow ${ }^{21}$ has suggested the use of certain empirical relationships between the various physical properties in order to systematize and group AO materials. It is well known that such relations exist, for example, for the refractive index and the acoustic velocity for such groups as the alkali halides, the mineral oxides, and the III-IV compounds.

A large amount of data has been collected on the refractive indices of crystals, and generally good agreement is found with the Gladstone-Dale ${ }^{22}$ equation

$$
\frac{n-1}{r}=\sum_{i} q_{i} E_{i}
$$

in which $R_{i}$ is the specific refraction of the $i$ th component and $q_{i}$ is percentage by weight. Reliable values of $R_{i}$ have been determined from mineralogical data over many years. From the expression for the $\mathrm{AO}$ figure of merit, it is apparent that a high value of refractive index is desirable for achieving high diffraction efficiency. It is not, however, possible simply to select for consideration those materials with high refractive index, as even a casual survey shows that such materials tend to be opaque at shorter wavelengths. This trend was examined in great detail by Wemple and DiDomenico, ${ }^{23}$ who found that the refractive index is simply related to the energy band gap. The semiempirical relation for oxide materials is

$$
n^{2}=1+\frac{15}{E_{g}}
$$

where $E_{g}$ is the energy gap (expressed in electron volts). For other classes of materials the energy gap constant will be different, but the same form holds. It can be seen from Equation 11.89 that the largest refractive index for an oxide material transparent over the entire visible range (cutoff wavelength at $0.4 \mu \mathrm{m}$ ) is 2.44 . Higher refractive indices can be chosen only by sacrificing transparency at short wavelengths. 
Pinnow ${ }^{21}$ has found that a good approximation to the acoustic velocity for a wide range of materials is obtained with the relation

$$
\log \left(\frac{n}{r}\right)=-b \bar{M}+d
$$

where $\bar{M}$ is the mean atomic weight, defined as the total molecular weight divided by the number of atoms per molecule, and $b$ and $d$ are constants. Large values of $d$ are generally associated with harder materials, while $b$ does not vary greatly for oxides. Thus, in general, low acoustic velocities tend to be found in materials of high density, as is intuitively expected. Another useful velocity relationship has been pointed out by Uchida and Niizeki; ${ }^{24}$ this is the Lindemann formula relating the melting temperature $T_{m}$ and the mean acoustic velocity $v_{m^{\prime}}$

$$
n_{m}^{2}=\frac{c T_{m}}{\bar{M}}
$$

in which $c$ is a constant dependent upon the material class. This relation suggests that high-efficiency materials would likely be found among those with large mean atomic weight and low melting temperature, that is, dense, soft materials.

In order for an $\mathrm{AO}$ material to be useful for wideband applications, the ultrasonic attenuation must be small at high frequencies. An attenuation that is often taken as an upper limit is $1 \mathrm{~d} B / \mu$ s (so that the useful aperture will depend upon the velocity). Many materials that might be highly efficient and otherwise suitable are excessively lossy at high frequency. A microscopic treatment of ultrasonic attenuation was carried out by Woodruff and Ehrenreich. ${ }^{25}$ Their formula for the ultrasonic attenuation is

$$
a=\frac{g^{2} \Omega^{2} k T}{r n^{5}}
$$

where $\Omega$ is the radian frequency, $\gamma$ is the Griinneisen constant, $\kappa$ is the thermal conductivity, and $T$ is the absolute temperature. This formula would suggest that the requirement of low acoustic velocity and low attenuation conflict with each other, since $\alpha \sim v^{-5}$; it is quite unusual for materials with low acoustic velocity to not also have a high absorption, at least for the low-velocity modes.

The determination of the photoelastic constants of materials is essentially an empirical study, although a microscopic theory of Mueller, ${ }^{26}$ developed for cubic and amorphous structures, is still referenced. For both ionic and covalent bonded materials the photoelastic effect derives from two mechanisms: the change of refractive index with density, and the change in index with polarizability under the strain. Both of these effects may have the same or opposite sign under a given strain, and one or the other may be the larger. It is for this reason that the magnitude or even the sign of the photoelastic constant cannot be predicted, since the effects may completely cancel each other. It is possible, however, to estimate the maximum constants for groups of materials. This has been done for three important groups with the result

$$
\left|P_{\max }\right|=\left\{\begin{array}{cc}
0.21 & \text { water-insolubeoxides } \\
0.35 & \text { water-solubeoxides } \\
0.20 & \text { alkalihalides }
\end{array}\right.
$$


In general, the photoelastic tensor components corresponding to shear strain will be less than those corresponding to compressional strain because there is no change, to first order, of density with shear; only the polarizability effect will be present. It is always possible that exceptionally large values of shear-related photoelastic coefficients may be found, but in no case could they be expected to be larger than the estimated value of $\left|P_{\max }\right|$. The maximum values of photoelastic constant are shown in Table 11.1 for a number of important oxides and other materials.

\subsubsection{Selected Materials for Acousto-Optic Scanners}

Among older materials, those that have been shown useful for $\mathrm{AO}$ applications are fused quartz, because of its excellent optical quality and low cost for large sizes, and sapphire and lithium niobate, because of their exceptionally low acoustic losses at microwave frequencies. For IR applications germanium ${ }^{27}$ has proven very useful, as has arsenic trisulfide glass, where bandwidth requirements are not high. Among the newer crystal materials, very good $\mathrm{AO}$ performance has been obtained in the visible with $\mathrm{GaP}^{28}$ and $\mathrm{PbMoO}_{4} \cdot{ }^{29,30}$ One of the most interesting new materials to be developed within the past several years is $\mathrm{TeO}_{2},{ }^{31}$ which along with $\mathrm{PbMoO}_{4}$ has found wide use in commercially available AO scanners. More design details for devices employing this material will be given later. Among the new materials that have been developed for IR applications, very high performance has been reached with several chalcogenide crystals. ${ }^{32}$ Particularly important members of this group of materials include $\mathrm{Tl}_{3} \mathrm{AsS}_{4}{ }^{33}$ and $\mathrm{Tl}_{3} \mathrm{PSe}_{4}{ }^{34}$ The compound $\mathrm{Tl}_{3} \mathrm{AsSe}_{3}{ }^{35}$ is particularly interesting beyond its possible use as an IR AOM material. Since $\mathrm{Tl}_{3} \mathrm{AsSe}_{3}$ belongs to the crystal class $3 \mathrm{~m}$, its symmetry permits it to possess a nonzero $p_{41}$ photoelastic coefficient, and it is suitable for use as a collinear tunable AO filter, a device first realized by Harris, ${ }^{36}$ using lithium niobate. Tables 11.2 through 11.4 summarize the properties of some

TABLE 11.1

Maximum Photoelastic Coefficients

\begin{tabular}{|c|c|}
\hline Material & $\left|P_{\max }\right|$ measured \\
\hline$\overline{\mathrm{LiNbO}_{3}}$ & 0.20 \\
\hline $\mathrm{TiO}_{2}$ & 0.17 \\
\hline $\mathrm{Al}_{2} \mathrm{O}_{3}$ & 0.25 \\
\hline $\mathrm{PbMoO}_{4}$ & 0.28 \\
\hline $\mathrm{TeO}_{2}$ & 0.23 \\
\hline $\mathrm{Sr}_{5} \mathrm{Ba}_{.5} \mathrm{Nb}_{2} \mathrm{O}_{6}$ & 0.23 \\
\hline $\mathrm{SiO}_{2}$ & 0.27 \\
\hline YIG & 0.07 \\
\hline $\mathrm{Ba}\left(\mathrm{NO}_{3}\right)_{2}$ & 0.35 \\
\hline$\alpha-\mathrm{HIO}_{3}$ & 0.50 \\
\hline $\mathrm{Pb}\left(\mathrm{NO}_{3}\right)_{2}$ & 0.60 \\
\hline ADP & 0.30 \\
\hline $\mathrm{CdS}$ & 0.14 \\
\hline GaAs & 0.16 \\
\hline $\mathrm{As}_{2} \mathrm{~S}_{3}$ & 0.30 \\
\hline
\end{tabular}


TABLE 11.2

Acousto-Optic Properties of Amorphous Materials

\begin{tabular}{|c|c|c|c|c|c|c|c|c|c|}
\hline Material & $\begin{array}{c}\text { Transmission } \\
\text { range }(\mu \mathrm{m})\end{array}$ & $\begin{array}{c}\text { Acoustic } \\
\text { mode }\end{array}$ & $\begin{array}{c}\nu \\
(\mathrm{cm} / \mathrm{s} \\
\left.\times 10^{5}\right)\end{array}$ & $\begin{array}{c}\Gamma(\mathrm{dB} / \\
\mathrm{cm} \\
\left.\mathrm{GHz}^{2}\right)\end{array}$ & $\begin{array}{l}\text { Opt. } \\
\text { pol. } \\
\text { dir. }\end{array}$ & $\begin{array}{c}n \\
(0.633 \\
\mu \mathrm{m})\end{array}$ & $\begin{array}{c}M_{1}\left(\mathrm{~cm}^{2}\right. \\
\mathrm{s} / \mathrm{g} \times \\
\left.10^{-7}\right)\end{array}$ & $\begin{array}{c}M_{2}\left(\mathrm{~s}^{3} / \mathrm{g}\right. \\
\left.\times 10^{-18}\right)\end{array}$ & $\begin{array}{c}M_{3}(\mathrm{~cm} \\
\mathrm{s}^{2} / \mathrm{g} \times \\
\left.10^{-12}\right)\end{array}$ \\
\hline Water & $0.2-0.9$ & $\mathrm{~L}$ & 1.49 & 2400 & $\|$ or $\perp$ & 1.33 & 37.2 & 126 & 25 \\
\hline $\begin{array}{l}\text { Fused } \\
\text { quartz }\end{array}$ & $0.2-4.5$ & L & 5.96 & 12 & & 1.46 & 8.05 & 1.56 & 1.35 \\
\hline SF-4 & $0.38-1.8$ & L & 3.63 & 220 & $\perp$ & 1.62 & 1.83 & 4.51 & 3.97 \\
\hline SF-59 & $0.46-2.5$ & L & 3.20 & 1200 & $\|$ or $\perp$ & 1.95 & 39 & 19 & 12 \\
\hline SF-58 & & $\mathrm{L}$ & 3.26 & 1200 & $\|$ or $\perp$ & 1.91 & 18.2 & 9 & 5.6 \\
\hline SF-57 & & $\mathrm{L}$ & 3.41 & 500 & $\|$ & 1.84 & 19.3 & 9 & 5.65 \\
\hline SF-6 & & L & 3.51 & 500 & $\|$ or $\perp$ & 1.80 & 15.5 & 7 & 4.42 \\
\hline $\mathrm{As}_{2} \mathrm{~S}_{3}$ & $0.6-11$ & $\mathrm{~L}$ & 2.6 & 170 & $\|$ & 2.61 & 762 & 433 & 293 \\
\hline $\mathrm{As}_{2} \mathrm{~S}_{5}$ & $0.5-10$ & L & 2.22 & & & 2.2 & 278 & 256 (est.) & 125 \\
\hline
\end{tabular}

TABLE 11.3

Acousto-Optic Properties of Crystals for the Visible

\begin{tabular}{|c|c|c|c|c|c|c|c|c|c|}
\hline Material & $\begin{array}{c}\text { Transmission } \\
\text { range }(\mu \mathrm{m})\end{array}$ & $\begin{array}{c}\text { Acoustic } \\
\text { mode \& } \\
\text { prop. dir. }\end{array}$ & $\begin{array}{c}\nu \\
(\mathrm{cm} / \mathrm{s} \\
\left.\times 10^{5}\right)\end{array}$ & $\begin{array}{c}\Gamma(\mathrm{dB} / \\
\mathrm{cm} \\
\left.\mathrm{GHz}^{2}\right)\end{array}$ & $\begin{array}{l}\text { Opt. pol. } \\
\text { dir. }\end{array}$ & $\begin{array}{c}n \\
(0.633 \\
\mu \mathrm{m})\end{array}$ & $\begin{array}{c}M_{1}\left(\mathrm{~cm}^{2}\right. \\
\mathrm{s} / \mathrm{g} \times \\
\left.10^{-7}\right)\end{array}$ & $\begin{array}{c}M_{2} \\
\left(\mathrm{~s}^{3} / \mathrm{g} \times\right. \\
\left.10^{-18}\right)\end{array}$ & $\begin{array}{c}M_{3}(\mathrm{~cm} \\
\mathrm{s}^{2} / \mathrm{g} \times \\
\left.10^{-12}\right)\end{array}$ \\
\hline \multirow{2}{*}{$\mathrm{LiNbO}_{3}$} & \multirow[t]{2}{*}{$0.04-4.5$} & L[100] & 6.57 & 0.15 & & 2.20 & 66.5 & 7.0 & 10.1 \\
\hline & & $\mathrm{S}[001]$ & 3.59 & 2.6 & $\perp$ & 2.29 & 9.2 & 2.92 & 2.4 \\
\hline $\mathrm{Al}_{2} \mathrm{O}_{3}$ & $0.15-6.5$ & L[100] & 11.0 & 0.2 & $\|$ & 1.77 & 7.7 & 0.36 & 0.7 \\
\hline \multirow[t]{2}{*}{ YAG } & \multirow[t]{2}{*}{$0.3-5.5$} & $\mathrm{~L}[100]$ & 8.60 & 0.25 & $\perp$ & 1.83 & 0.98 & 0.073 & 0.114 \\
\hline & & S[100] & 5.03 & 1.1 & $\|$ or $\perp$ & 1.83 & 1.1 & 0.25 & 0.23 \\
\hline $\mathrm{TiO}_{2}$ & $0.45-6$ & $\mathrm{~L}[001]$ & 10.3 & 0.55 & $\perp$ & 2.58 & 44 & 1.52 & 4 \\
\hline \multirow[t]{2}{*}{$\mathrm{SiO}_{2}$} & \multirow[t]{2}{*}{$0.12-4.5$} & L[001] & 6.32 & 2.1 & $\perp$ & 1.54 & 9.11 & 1.48 & 1.44 \\
\hline & & $\mathrm{L}[100]$ & 5.72 & 3.0 & [001] & 1.55 & 12.1 & 2.38 & 2.11 \\
\hline \multirow{4}{*}{$\begin{array}{l}\alpha-\mathrm{HIO}_{3} \\
\mathrm{PbMoO}_{4} \\
\mathrm{TeO}_{2}\end{array}$} & \multirow[t]{2}{*}{$0.3-1.8$} & L[001] & 2.44 & 10 & [100] & 1.99 & 103 & 86 & 42 \\
\hline & & L[001] & 3.63 & 15 & $\|$ & 2.62 & 108 & 36.3 & 29.8 \\
\hline & \multirow[t]{2}{*}{$0.35-5$} & $\mathrm{~L}[001]$ & 4.20 & 15 & $\perp$ & 2.26 & 138 & 34.5 & 32.8 \\
\hline & & $\mathrm{S}[110]$ & 0.616 & 90 & Circ [001] & 2.26 & 68.0 & 793 & 110 \\
\hline $\mathrm{Pb}_{2} \mathrm{MoO}_{5}$ & $0.4-5$ & La-axis & 2.96 & 25 & b-axis & 2.183 & 242 & 127 & 82 \\
\hline
\end{tabular}

of the materials that have been studied for $\mathrm{AO}$ applications. The acoustic attenuation constant in these tables is defined as

$$
\Gamma=\frac{a}{f^{2}}
$$

which supposes that the attenuation increases quadratically with frequency. This will be the case for good-quality single crystals, but not for polycrystalline, highly impure, or amorphous materials. For the latter, the constant given in the tables is a rough estimate, based on measurements at the higher frequencies. The light polarization direction is designated as parallel or perpendicular according to whether the light polarization is parallel or perpendicular to the acoustic beam direction. Table 11.2 lists some of the more 
TABLE 11.4

Acousto-Optic Properties of Infrared Crystals

\begin{tabular}{|c|c|c|c|c|c|c|c|c|c|c|}
\hline Material & $\begin{array}{c}\text { Transmission } \\
\text { range }(\mu \mathrm{m})\end{array}$ & $\begin{array}{c}\text { Acoustic } \\
\text { mode \& } \\
\text { prop. div. }\end{array}$ & $\begin{array}{c}\nu \\
(\mathrm{cm} / \mathrm{s} \\
\left.\times 10^{5}\right)\end{array}$ & $\begin{array}{c}\Gamma(\mathrm{dB} / \\
\mathrm{cm} \\
\left.\mathrm{GHz}^{2}\right)\end{array}$ & $\begin{array}{l}\text { Opt. } \\
\text { pol. } \\
\text { dir. }\end{array}$ & $\begin{array}{c}\lambda \\
(\mu \mathrm{m})\end{array}$ & $n$ & $\begin{array}{c}M_{1}\left(\mathrm{~cm}^{2}\right. \\
\mathrm{s} / \mathrm{g} \times \\
\left.10^{-7}\right)\end{array}$ & $\begin{array}{c}M_{2} \\
\left(s^{3} / g \times\right. \\
\left.10^{-18}\right)\end{array}$ & $\begin{array}{c}M_{3}(\mathrm{~cm} \\
\mathrm{s}^{2} / \mathrm{g} \times \\
\left.10^{-12}\right)\end{array}$ \\
\hline \multirow[t]{2}{*}{$\overline{\mathrm{Ge}}$} & $2-020$ & $\mathrm{~L}[111]$ & 5.50 & 30 & $\|$ & 10.6 & 4.00 & 10,200 & 840 & 1850 \\
\hline & & S[100] & 3.51 & 9 & $\|$ or $\perp$ & 10.6 & 4.00 & 1430 & 290 & 400 \\
\hline $\mathrm{Tl}_{3} \mathrm{AsS}_{4}$ & $0.6-12$ & L[001] & 2.5 & 29 & $\|$ & 1.15 & 2.63 & 620 & 510 & 290 \\
\hline \multirow[t]{2}{*}{ GaAs } & $1-11$ & L[110] & 5.15 & 30 & $\|$ & 1.15 & 3.37 & 925 & 104 & 179 \\
\hline & & S[100] & 3.32 & & or $\perp$ & 1.15 & 3.37 & 155 & 46 & 49 \\
\hline $\mathrm{Ag}_{3} \mathrm{AsS}_{3}$ & $0.6-13.5$ & L[001] & 2.65 & 800 & $\|$ & .633 & 2.98 & 816 & 390 & 308 \\
\hline $\mathrm{Tl}_{3} \mathrm{AsSe}_{3}$ & $1.25-18$ & L[100] & 2.15 & 314 & $\perp$ & 3.39 & 3.15 & 654 & 445 & 303 \\
\hline $\mathrm{Tl}_{3} \mathrm{PSe}_{4}$ & $0.85-9$ & L[100] & 2.0 & 150 & $\|$ & 1.15 & 2.9 & 2866 & 2069 & 1288 \\
\hline $\mathrm{TlGaSe}_{2}$ & $0.6-20$ & L[001] & 2.67 & 240 & $\|$ & .633 & 2.9 & 430 & 393 & 161 \\
\hline CdS & $0.5-11$ & $\mathrm{~L}[100]$ & 4.17 & 90 & $\|$ & .633 & 2.44 & 52 & 12 & 12 \\
\hline ZnTe & $0.55-20$ & L[110] & 3.37 & 130 & $\|$ & 1.15 & 2.77 & 75 & 18 & 19 \\
\hline $\mathrm{GaP}$ & $0.6-10$ & L[110] & 6.32 & 6.0 & & .633 & 3.31 & 75 & 30 & 71 \\
\hline \multirow[t]{2}{*}{$\mathrm{ZnS}$} & $0.4-12$ & $\mathrm{~L}[001]$ & 5.82 & 27 & $\|$ & .633 & 2.35 & 27 & 3.4 & 4.7 \\
\hline & & $S[001]$ & 2.63 & 130 & & .633 & 2.35 & 14 & 8.4 & 5.2 \\
\hline $\mathrm{Te}$ & $5-20$ & L[100] & 2.2 & 60 & $\|$ & 10.6 & 4.8 & 10,200 & 4400 & 4640 \\
\hline
\end{tabular}

important amorphous materials, which may be useful if large sizes are desired or very low cost is required, but none of which can be used at frequencies much above $30 \mathrm{MHz}$. Table 11.3 lists the most important class of materials, crystals that are transparent throughout the visible with very low acoustic losses. Table 11.4 lists high-efficiency crystal materials that are transparent in the IR and have reasonably low acoustic losses.

An overall summary of a few outstanding (in one or another respect) selected AO materials presented in these tables is shown in Figure 11.19. Using figure of merit and acoustic attenuation as criteria of quality, it is clear that a trade-off between these two parameters exists, and that the selection of the optimum material will be determined by the system requirements.

\subsection{ACOUSTIC TRANSDUCER DESIGN}

\subsubsection{Transducer Characteristics}

The second key component of the AO scanner after the optical medium, is the transducer structure, which includes the piezoelectric layer, bonding films, backing layers, and matching network. Recent advances in this area have made available a number of new piezoelectric materials of very high electromechanical conversion efficiency, and bonding techniques that permit this high conversion efficiency to be maintained over a large bandwidth. Furthermore, the design of high-performance transducer structures utilizing this new technology has been facilitated by new analytical tools $\mathrm{s}^{37,38}$ that lend themselves to computer programs for optimizing this performance.

The most elementary configuration of a thickness-driven transducer structure is shown in Figure 11.20. It consists of the piezoelectric layer, thin film or plate, excited by metallic electrodes on both faces, and a bonding layer to acoustically couple the piezoelectric to the 


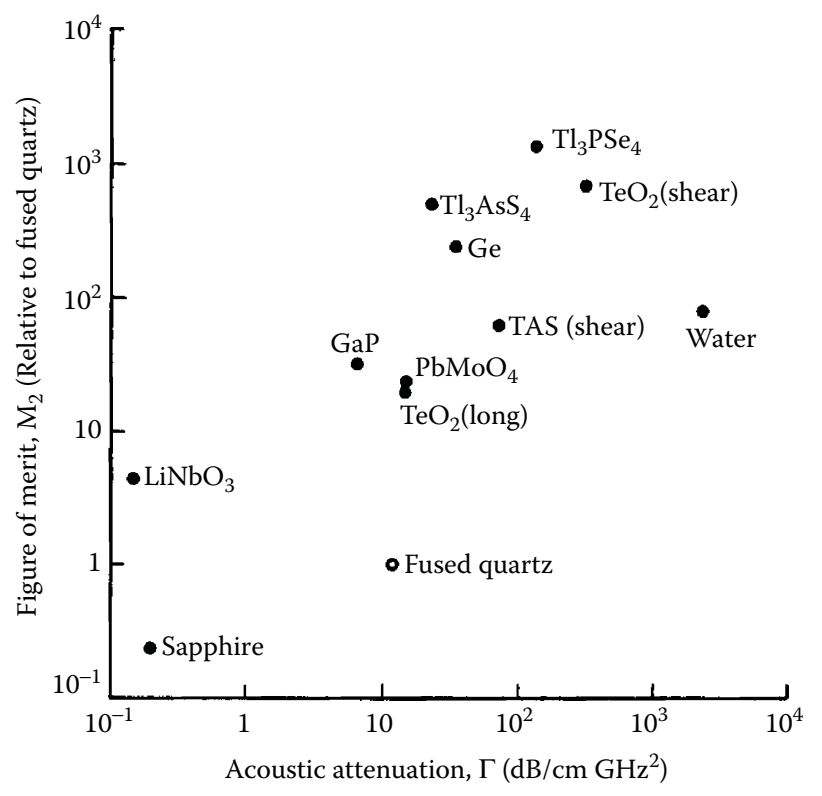

FIGURE 11.19

Figure of merit versus acoustic attenuation.

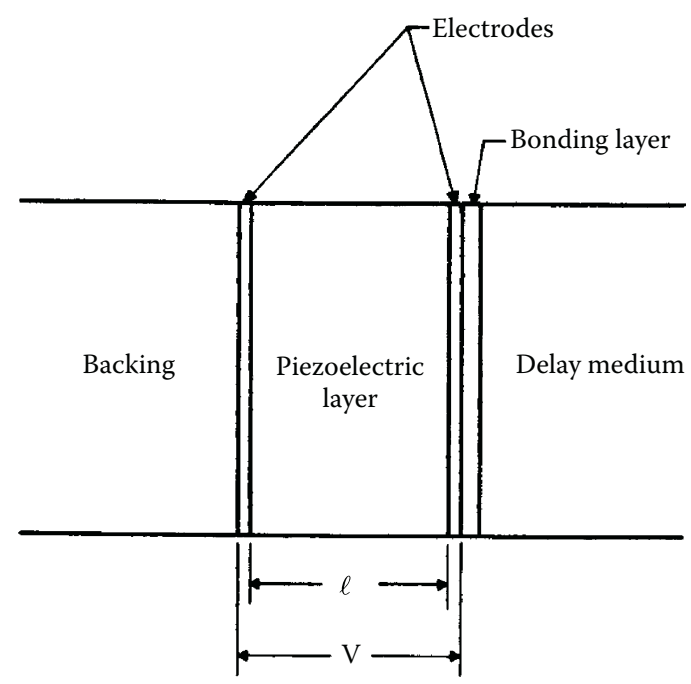

FIGURE 11.20

Transducer structure.

delay medium, or optical crystal. The backing is applied to mechanically load the transducer for bandwidth adjustment but may simply be left as air. The thickness of the transducer is about half an acoustic wavelength at the resonant frequency, and the thickness of the bonding layer is chosen to allow high, broadband acoustic transmission. The most efficient operation of the transducer is obtained when the mechanical impedances of all 
the layers are equal. The mechanical impedance is

$$
Z=r u
$$

and in general there is not sufficient choice of available materials to satisfy this condition. When the impedances are unequal, reflection occurs at the interfaces, reducing the efficiency of energy transfer. The reflection and transmission coefficients at the boundary between two media of impedances $Z_{1}$ and $Z_{2}$ are

$$
\begin{aligned}
& R=\frac{\left(Z_{1}-Z_{2}\right)^{2}}{\left(Z_{1}+Z_{2}\right)^{2}} \\
& T=\frac{4 Z_{1} Z_{2}}{\left(Z_{1}+Z_{2}\right)^{2}}
\end{aligned}
$$

The electromechanical analysis is generally carried out in terms of an equivalent circuit model, first proposed by Mason. ${ }^{39}$ Several variations of the equivalent circuit have since been developed, but the one due to Mason is shown in Figure 11.21. The fundamental constants of the transducer are permittivity $s$, acoustic velocity $v$, and electromechanical coupling factor $k$. The other parameters are transducer thickness $l$ and area $S$. With these parameters, the circuit components shown in Figure 11.21 are

$$
\begin{gathered}
C_{0}=\in \frac{S}{l} \\
f=k\left(\frac{1}{p} w_{0} c_{0} Z_{0}\right) \frac{1}{2} \\
Z_{A}=j Z_{0} \tan \frac{g}{2}
\end{gathered}
$$

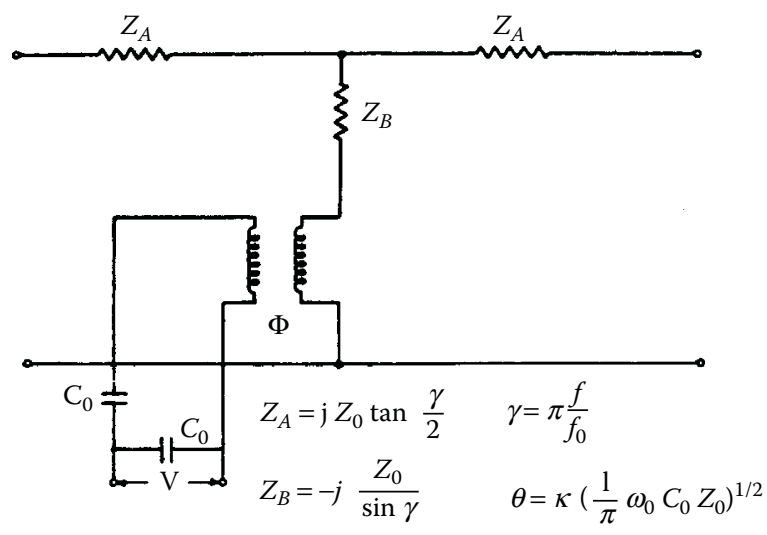

FIGURE 11.21

Equivalent circuit of Mason. 


$$
Z_{B}=-j \frac{Z_{0}}{\sin g}
$$

where

$$
\begin{aligned}
& w_{0}=\frac{p u}{l} \\
& g=p \frac{w}{w_{0}} \\
& Z_{0}=\operatorname{Sru}
\end{aligned}
$$

This equivalent circuit was used by Sittig ${ }^{37}$ and Meitzler and Sittig ${ }^{38}$ to analyze the propagation characteristics of acoustic energy between a piezoelectric and a delay medium. This was done in terms of a two-port electromechanical network, described by the chain matrix

$$
\left(\begin{array}{ll}
A & B \\
C & D
\end{array}\right)=\prod_{m}\left(\begin{array}{ll}
A_{m} & B_{m} \\
C_{m} & D_{m}
\end{array}\right)
$$

If the equivalent circuit of Figure 11.21 is terminated at the input with a voltage source $V_{s}$ and impedance $Z_{s}$, and at the output with a transmission medium of mechanical impedance $Z_{t}$, output voltage $V_{l}$, and load impedance $Z_{l}$, as shown in Figure 11.22, then the insertion loss is

$$
L=20 \log \frac{V_{s}}{V_{l}}+20 \log \left|\frac{Z_{s}+Z_{t}}{Z_{l}}\right| \mathrm{dB}
$$

The impedances $Z_{s}$ and $Z_{t}$ are assumed to be purely resistive and

$$
\frac{V_{l}}{V_{s}}=\frac{2 Z_{l} Z_{t}}{\left\{A Z_{t}+B+Z_{s}\left(C Z_{t}+D\right)\right\}\left\{A Z_{t}+B+Z_{t}\left(C Z_{t}+D\right)\right\}}
$$

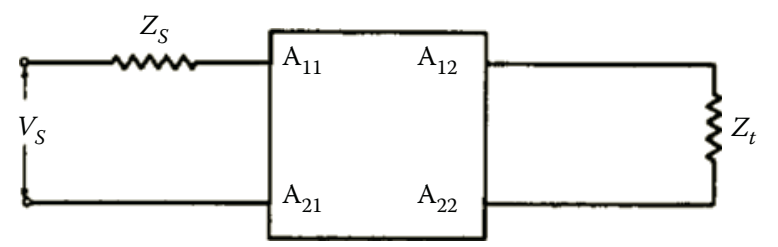

FIGURE 11.22

Terminated two-port transducer. 
The two-port transfer matrix was obtained by Sittig, ${ }^{40}$ with the result

$$
A=\frac{1}{f H}\left|\begin{array}{ll}
A^{\prime} & B^{\prime} \\
C^{\prime} & D^{\prime}
\end{array}\right| \begin{array}{cc}
\cos g+j z_{\mathrm{b}} \sin g & Z_{0}\left(Z_{\mathrm{b}} \cos g+z \sin g\right) \\
\frac{j \sin g}{z_{0}} & 2(\cos g-1)+j z_{b} \sin g
\end{array} \mid
$$

where

$$
z_{b}=\frac{z_{b}}{z_{0}}, \quad H=\cos g-1+j Z_{b} \sin g
$$

and

$$
A^{\prime}=1, \quad B^{\prime}=j \frac{f^{2}}{{ }_{w} C_{0}}, C^{\prime}=j w C_{0}, D^{\prime}=0
$$

The impedance $Z_{b}$ represents the mechanical impedance of layers placed on the back surface of the transducer for loading, $Z_{b}=S \rho_{b} v_{b}$. In case the transducer is simply air-backed, $Z_{b} \quad 0$. Electrical matching may be done at the input network by adding inductors either in parallel or in series in order to be electrically resonant with the transducer capacity $C_{0}$ at midband, $\omega=\omega_{0}$. If no inductances are added, the minimum loss condition is achieved for

$$
R_{s}=\frac{1}{W_{0} C_{0}}
$$

where $R_{s}$ is the source resistance. The inductance, if added, is chosen so that

$$
L=\frac{1}{w_{0}^{2} C_{0}}
$$

A result of the matrix analysis shows that when piezoelectric materials with large values of the coupling constant $\kappa$ are used, it is possible to achieve large fractional bandwidths without the necessity for electrical matching networks. As an example of the results obtained with this formalism, several plots of the frequency dependence of transducer loss for different values of the coupling constant are shown in Figure 11.23.

\subsubsection{Transducer Materials}

The piezoelectric material itself is perhaps the single most important factor governing the efficiency with which electrical energy can be converted to acoustic energy, this through the electromechanical coupling factor $\kappa$. The coupling efficiency is equal to $\kappa$. Prior to the discovery of lithium niobate, quartz was the most commonly used high-frequency transducer material, although its coupling factor, even for the most efficient crystal orientations, is rather small. The very-high-efficiency transducers were introduced with the discovery of various new ferroelectrics, such as lithium niobate, lithium tantalate, and the ceramic 


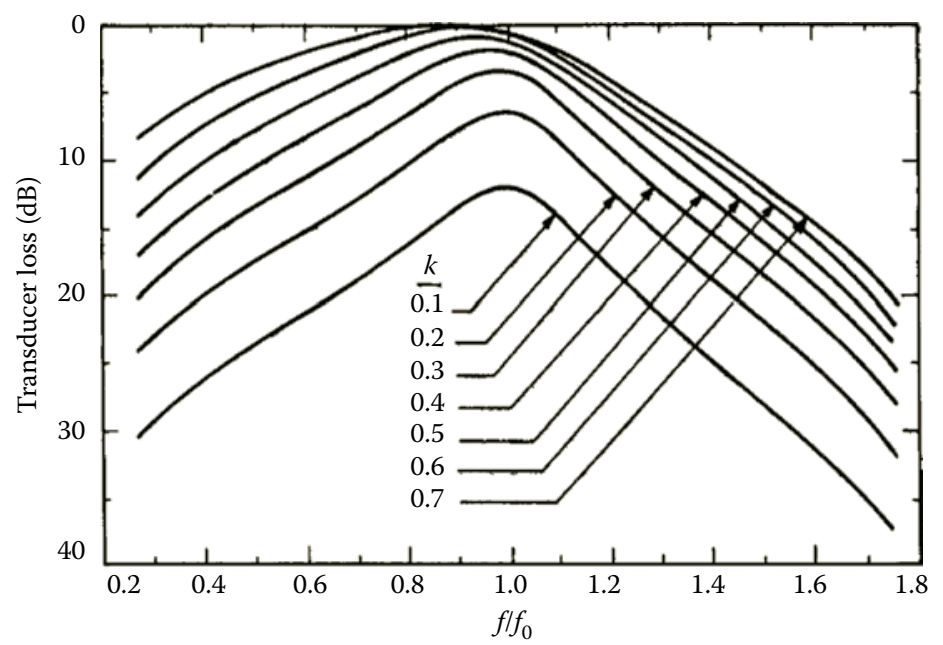

FIGURE 11.23

Transducer loss for various values of $\kappa ; z_{0 t}=0.4$ and $R_{s}=\left(\omega_{0} C_{0}\right)^{-1}$.

PZT materials, lead-titanate-zirconate. While the PZT transducers have among the highest values of $\kappa$, up to 0.7 , they are not suitable for high-frequency applications since they cannot be polished to very thin plates. The most suitable piezoelectric transducer materials for high-frequency applications and their important properties are listed in Table 11.5, which is based on a compilation of Meitzler. ${ }^{41}$ In order to produce transducers in the high-frequency range, say larger than $100 \mathrm{MHz}$, the piezoelectric crystal must be very thin $(<20-30 \mu \mathrm{m})$. There are three well-established techniques for fabricating such thin transducers. In the first method, the piezoelectric plate is lapped to the desired thickness by the usual optical shop methods and then bonded to the delay medium. This method becomes impossibly difficult for transducers of even small area as their frequency increases, because such thin plates cannot be manipulated. A much more convenient technique is to bond the piezoelectric plates with a convenient thickness, say several tenths of a millimeter, to the delay medium and then lap the plate to the final thickness. In both methods, one electrode is first deposited on the delay medium, and in the case of thinning the piezoelectric after bonding, the second electrode and back layers are deposited as the final step. Care is required in lapping the bonded transducer so that the base electrode is not damaged by the polishing compound. If a chemically active compound, such as Cyton, is used, the delay medium as well as the electrode may be attacked and must be protected by some appropriate coating, such as photoresist. The final electrical connection to the top electrode must be made in some fashion that does not mass-load the transducer and distort its bandpass characteristics, or be so small as to cause hot spots from high current densities. The usual method is to bond thin gold wire or ribbon onto electrode tabs, as is done for electronic circuit chips. The most successful method for fabricating very-high-frequency transducers for longitudinal wave generation is by deposition of thin films of piezoelectric materials by methods that yield a desired crystallographic orientation. ${ }^{42,43}$ The materials used are $\mathrm{CdS}$ and $\mathrm{ZnO}$, whose properties are shown in Table 11.5.

Such piezoelectric thin films generally cannot be grown with values of $\kappa$ as high as that of the bulk material, but in the best circumstances $\kappa$ may approach $90 \%$. Thin-film transducers with band center frequencies up to $5 \mathrm{GHz}$ can be prepared by these techniques. 
TABLE 11.5

Properties of Transducer Materials

\begin{tabular}{|c|c|c|c|c|c|c|c|}
\hline Material & Density & Mode & Orientation & $K$ & $\varepsilon_{\text {rel }}$ & $v(\mathrm{~cm} / \mathrm{s})$ & $\mathrm{Z}\left(\mathrm{g} / \mathrm{s} \mathrm{cm}^{2}\right)$ \\
\hline \multirow[t]{3}{*}{$\mathrm{LiNbO}_{3}$} & 4.64 & $\mathrm{~L}$ & $36^{\circ} \mathrm{Y}$ & 0.49 & 38.6 & $7.4 \times 10^{5}$ & $34.3 \times 10^{5}$ \\
\hline & & S & $163^{\circ} \mathrm{Y}$ & 0.62 & 42.9 & $4.56 \times 10^{5}$ & $21.2 \times 10^{5}$ \\
\hline & & S & $X$ & 0.68 & 44.3 & $4.8 \times 10^{5}$ & $22.3 \times 10^{5}$ \\
\hline \multirow[t]{2}{*}{$\mathrm{LiTaO}_{3}$} & 7.45 & $\mathrm{~L}$ & $47^{\circ} \mathrm{Y}$ & 0.29 & 42.7 & $7.4 \times 10^{5}$ & $55.2 \times 10^{5}$ \\
\hline & & $S$ & $x$ & 0.44 & 42.6 & $4.2 \times 10^{5}$ & $31.4 \times 10^{5}$ \\
\hline \multirow[t]{2}{*}{$\mathrm{LiIO}_{3}$} & 4.5 & $\mathrm{~L}$ & $\mathrm{Z}$ & 0.51 & 6 & $2.5 \times 10^{5}$ & $11.3 \times 10^{5}$ \\
\hline & & $S$ & $\mathrm{Y}$ & 0.6 & 8 & $2.5 \times 10^{5}$ & $11.3 \times 10^{5}$ \\
\hline \multirow[t]{2}{*}{$\mathrm{Ba}_{2} \mathrm{NaNb}_{5} \mathrm{Oi}_{5}$} & 5.41 & $\mathrm{~L}$ & Z & 0.57 & 32 & $6.2 \times 10^{5}$ & $33.3 \times 10^{5}$ \\
\hline & & S & Y & 0.25 & 227 & $3.7 \times 10^{5}$ & $19.8 \times 10^{5}$ \\
\hline $\mathrm{LiGeO}_{2}$ & 4.19 & $\mathrm{~L}$ & Z & 0.30 & 8.5 & $6.3 \times 10^{5}$ & $26.2 \times 10^{5}$ \\
\hline $\mathrm{LiGeO}_{3}$ & 3.50 & $\mathrm{~L}$ & $\mathrm{Z}$ & 0.31 & 12.1 & $6.5 \times 10^{5}$ & $22.8 \times 10^{5}$ \\
\hline \multirow[t]{2}{*}{$\alpha \mathrm{SiO}_{2}$} & 2.65 & $\mathrm{~L}$ & $x$ & 0.098 & 4.58 & $5.7 \times 10^{5}$ & $15.2 \times 10^{5}$ \\
\hline & & S & Y & 0.137 & 4.58 & $3.8 \times 10^{5}$ & $10.2 \times 10^{5}$ \\
\hline \multirow[t]{3}{*}{$\mathrm{ZnO}$} & 5.68 & $\mathrm{~L}$ & $\mathrm{Z}$ & 0.27 & 8.8 & $6.4 \times 10^{5}$ & $36.2 \times 10^{5}$ \\
\hline & & S & $39^{\circ} \mathrm{Y}$ & 0.35 & 8.6 & $3.2 \times 10^{5}$ & $18.4 \times 10^{5}$ \\
\hline & & S & $Y$ & 0.31 & 8.3 & $2.9 \times 10^{5}$ & $16.4 \times 10^{5}$ \\
\hline \multirow[t]{2}{*}{$\mathrm{CdS}$} & 4.82 & $\mathrm{~L}$ & $\mathrm{Z}$ & 0.15 & 9.5 & $4.5 \times 10^{5}$ & $21.7 \times 10^{5}$ \\
\hline & & S & $40^{\circ} \mathrm{Y}$ & 0.21 & 9.3 & $2.1 \times 10^{5}$ & $10.1 \times 10^{5}$ \\
\hline \multirow[t]{2}{*}{$\mathrm{Bi}_{12} \mathrm{GeO}_{20}$} & 9.22 & $\mathrm{~L}$ & (111) & 0.19 & 38.6 & $3.3 \times 10^{5}$ & $30.4 \times 10^{5}$ \\
\hline & & S & (110) & 0.32 & 38.6 & $1.8 \times 10^{5}$ & $16.2 \times 10^{5}$ \\
\hline AIN & 3.26 & $\mathrm{~L}$ & $\mathrm{Z}$ & 0.20 & 8.5 & $10.4 \times 10^{5}$ & $34.0 \times 10^{5}$ \\
\hline
\end{tabular}

A problem that arises with large-area transducers, or even with small-area transducers at very high frequencies, is that of matching the electrical impedance to the source impedance. It is especially true for the ferroelectric, piezoelectric materials of very high dielectric constant that the impedance of the transducer may be so low that it becomes difficult to efficiently couple electrical power from the source to the transducer. This problem can be largely overcome by dividing the transducer into a series connected mosaic, as reported by Weinert and de Klerk. ${ }^{44}$ A schematic representation of such a mosaic transducer is shown in Figure 11.24. If a transducer of given area is divided into $N$ elements, which are connected in series, the capacity of the transducer will be reduced by a factor of $N^{2}$. As an example, a 1-GHz lithium niobate transducer of $0.25 \mathrm{~cm}^{2}\left(0.4 \mathrm{in}^{2}\right)$ area would represent a capacitive impedance of only $0.038 \Omega$; if this area were divided into a 16-element mosaic, the impedance would be increased to $10 \Omega$. A 40-element thin-film transducer is shown in Figure 11.25. The same considerations will apply at lower frequencies for transducers with large areas, about $1 \mathrm{~cm}^{2}$ or more. Because most ferroelectric transducer materials, such as the PZTs or lithium niobate, have high dielectric constants, the large areas will lead to very large capacitance values for frequencies far below $100 \mathrm{MHz}$. Thus, large-area transducers are usually divided into multiple elements, which are then wired in series to obtain the desired 50- $\Omega$ impedance to match to the RF driver. A largearea transducer that has been so wired is shown in Figure 11.26.

\subsubsection{Array Transducers}

One of the serious limitations of normal (i.e., isotropic) Bragg AOD is that imposed by the bandwidth as limited by the Bragg interaction. The most straightforward method of 


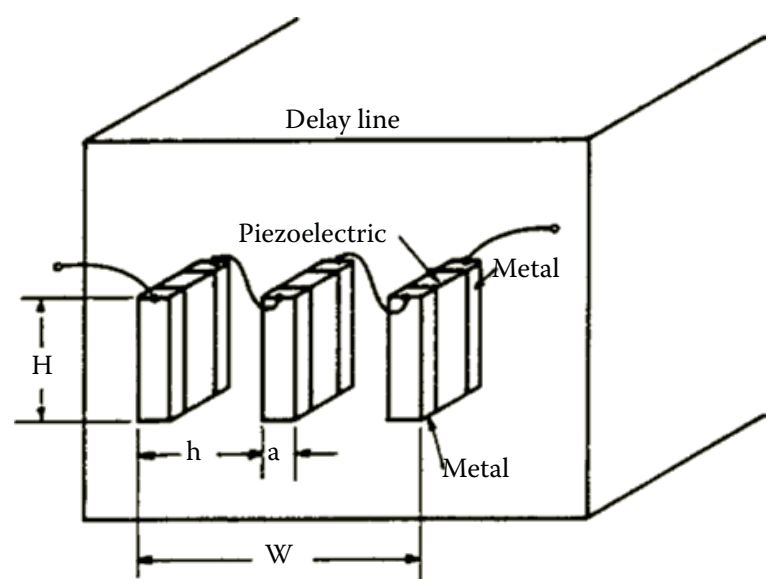

FIGURE 11.24

Schematic of mosaic transducer.

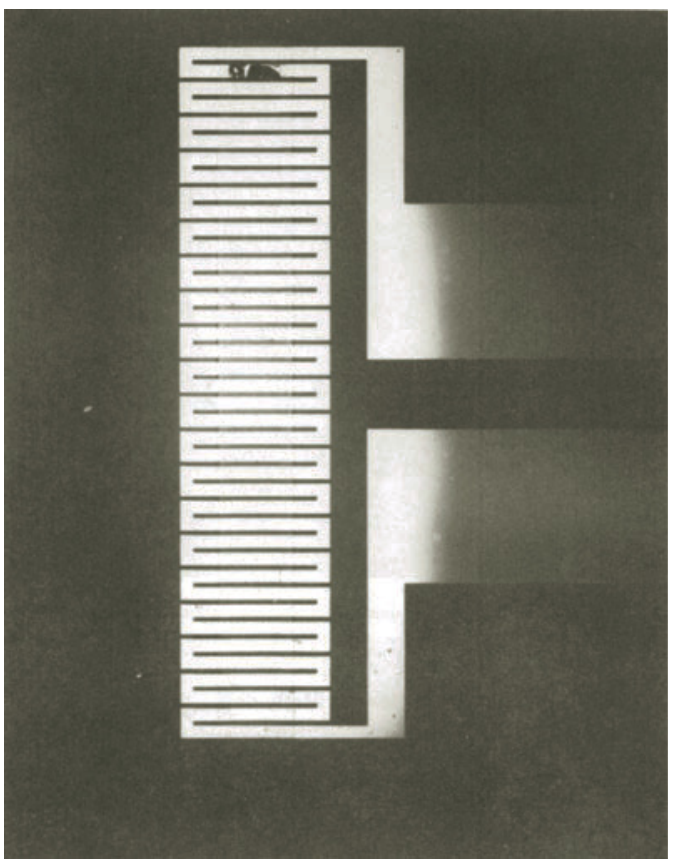

\section{FIGURE 11.25}

Forty-element thin-film mosaic transducer array.

enlarging the interaction bandwidth is simply to shorten the interaction length in order to increase the acoustic beam diffraction spread. This is generally not a very desirable method to increase bandwidth for systems in which the light to the Bragg cells is collimated because it wastes acoustic power; only those momentum components of the acoustic beam that can be phase matched to incident and diffracted light momentum components are useful. Furthermore, as the interaction length shrinks, the transducer becomes increasingly narrow, with a corresponding increase in power density. This increase in power 


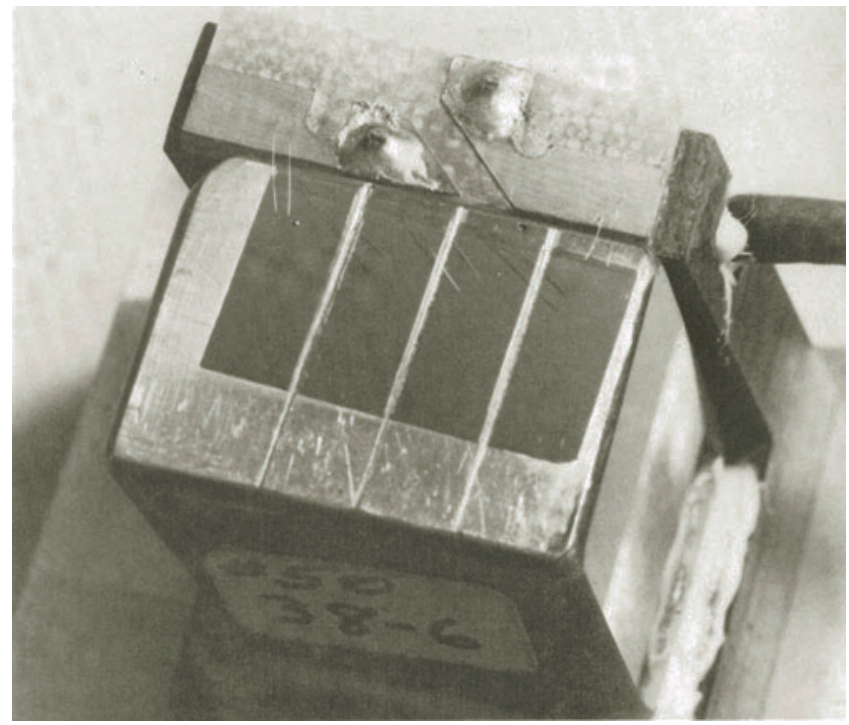

FIGURE 11.26

Four-element, series-connected lithium niobate transducer metal-bonded to Bragg cell.

density may produce heating at the transducer, which can cause thermal distortion in the deflector due to gradients in the acoustic velocity and refractive index.

An ideal solution to this difficulty would be one in which the acoustic beam changes in direction as the frequency is changed, so that for every frequency the Bragg angle is perfectly matched. The first approximation to such acoustic beam steering was carried out by Korpel ${ }^{45}$ for a television display system. This transducer consisted of a stepped array, as shown in Figure 11.27. The height of each step is one-half an acoustic wavelength at the band center $\Lambda_{0} / 2$, and the spacing $s$ between elements is chosen so as to optimize the tracking of the Bragg angle. Each element is driven $\pi$ rad out of phase with respect to the adjacent elements, and the net effect of such a transducer is to generate an acoustic wave with corrugated wavefronts, which are tilted at an angle with respect to the transducer surfaces when the frequency differs from the band center frequency $f_{0}$. For this transducer configuration, the acoustic beam steers with frequency but matches the Bragg angle only imperfectly.

To understand the steering properties of such an acoustic array, which was analyzed in detail by Coquin et al., ${ }^{45}$ consider the somewhat simpler arrangement shown in Figure 11.28 , in which each transducer element is driven $\Psi$ rad out of phase with respect to the next one, and $\Psi$ may be electrically varied. This causes the effective wavefront to be tilted by an angle $\theta_{e}$ with respect to the piecewise wavefronts radiating from the individual elements. If $\theta_{e}$ is small, it can be approximated by

$$
q_{e} \approx \tan q_{e}=\frac{\Psi}{2 p} \frac{\Lambda}{s}=\frac{\Psi}{K s}
$$

If the incident light beam makes an angle $\theta_{0}$ with the plane of the transducer and if the Bragg angle is $\theta_{B}=K / 2 k$, then the angular error from perfect matching is

$$
\Delta q=\left(q_{0}-q_{e}\right)-q_{B}=\left(q_{0}-\frac{\Psi}{K s}\right)-\frac{K}{2 k}
$$




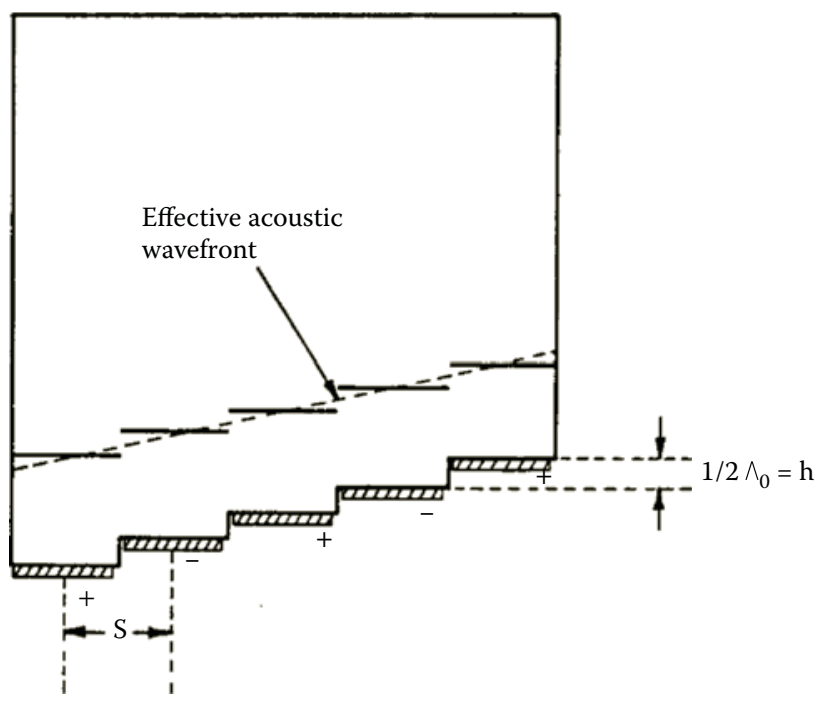

FIGURE 11.27

Stepped transducer array.

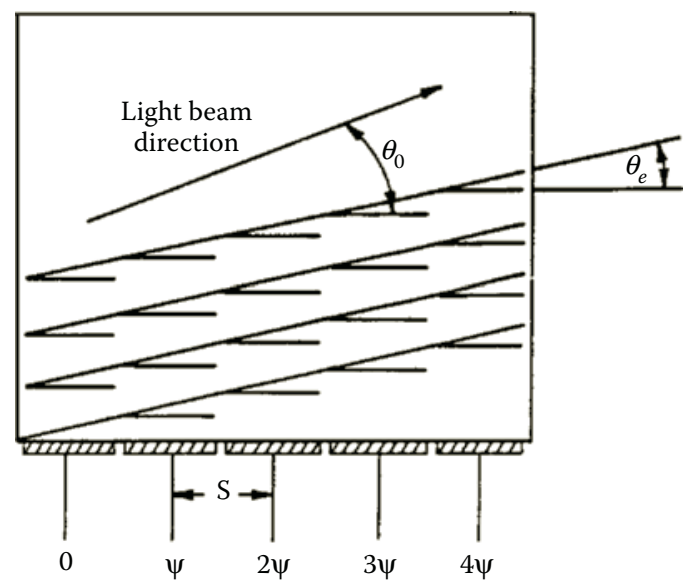

FIGURE 11.28

Steering of an acoustic beam by a phased array transducer.

The condition for perfect beam steering is that $\Delta \theta=0$ for all values of $K$; setting $\Delta \theta=0$, the required phase for perfect beam steering is

$$
\Psi_{P}=q_{0} K s-\frac{K^{2}}{2 k} s
$$

from which it can be seen that the phase must be a quadratic function of the acoustic frequency.

Most of the work done on acoustic beam steering has involved making various approximations to this condition. One such approximation is obtained by making $\Psi$ a linear function of frequency, with $\Psi=0$ at $f_{0}$, the midband frequency. This was accomplished in the 
step transducer method of Figure 11.27, as described in Ref. 46. For this case, the angle that the effective wavefronts make with respect to the transducer plane is

$$
q_{e} \approx \frac{p}{K s}-\frac{h}{s}
$$

where $h$ is the step height, and there is $180^{\circ}$ phase shift between adjacent elements. The resulting beam steering error is

$$
\Delta q=\left(q_{0}-\frac{K}{2 k}\right)+\left(\frac{h}{s}-\frac{p}{K s}\right)
$$

which can be made zero at the midband frequency $f_{0}$ by choosing

$$
\begin{aligned}
& h=\frac{1}{2} \Lambda_{0} \\
& s=\frac{\Lambda_{0}^{2}}{I}
\end{aligned}
$$

and

$$
q_{0}=\frac{1}{2} \frac{1}{\Lambda_{0}}
$$

A further improvement can be achieved by noting from Equation 11.115 that $\theta_{e}$ varies as $1 / f$, whereas perfect beam steering should lead to a linear variation of $\theta_{e}$ with $f$. Therefore, the constants $h, s$, and $\theta_{e}$ may be chosen to agree with the perfect beam steering case at two frequencies, rather than only one, as shown in Figure 11.29. This first-order beam steering can yield substantial improvements in performance for systems requiring less than oneoctave bandwidth, ${ }^{47}$ but bandwidths larger than this require a better approximation to the quadratic dependence of the phase on the acoustic frequency. The next higher approximation to perfect beam steering was carried out by Coquin et al. ${ }^{45}$ for a ten-element array, as shown in Figure 11.30. If the phase applied to each transducer corresponds to that for perfect steering, $\Psi_{1}=l \Psi_{p}$, and the element spacing is $s=\Lambda_{0}^{2} / 1$, the bandwidth extends from 0 to about $1.6 f_{0}$, the high-frequency drop-off being determined by the finite element spacing. Coquin pointed out that the deflector performance is very tolerant of errors in the individual phases; for example, if the phase applied to each transducer is within $45^{\circ}$ of the perfect beam steering phase, there is a loss of only $0.8 \mathrm{~dB}$ in diffracted light intensity. If the phase error is increased to $90^{\circ}$, the loss increases to $3 \mathrm{~dB}$. Thus, for deflectors, in which this degree of ripple is permissible, the transducer array may be driven by logic circuitry that sets digital phase shifters. This requires prior knowledge of the input frequency, or analog phase shifters, which accomplish the same function without the need for logic circuits.

An entirely different approach to broadband Bragg AO interaction matching is the use of the tilted transducer array, first reported by Eschler. ${ }^{48}$ A tilted transducer array consists of two or more transducers electrically connected in parallel and tilted in angle with respect to each other, as illustrated in Figure 11.31. Each transducer element in the array is designed to cover some fraction of the entire bandwidth, and its angle with respect to the incident light direction is chosen to match the Bragg angle at the center of its subband. 


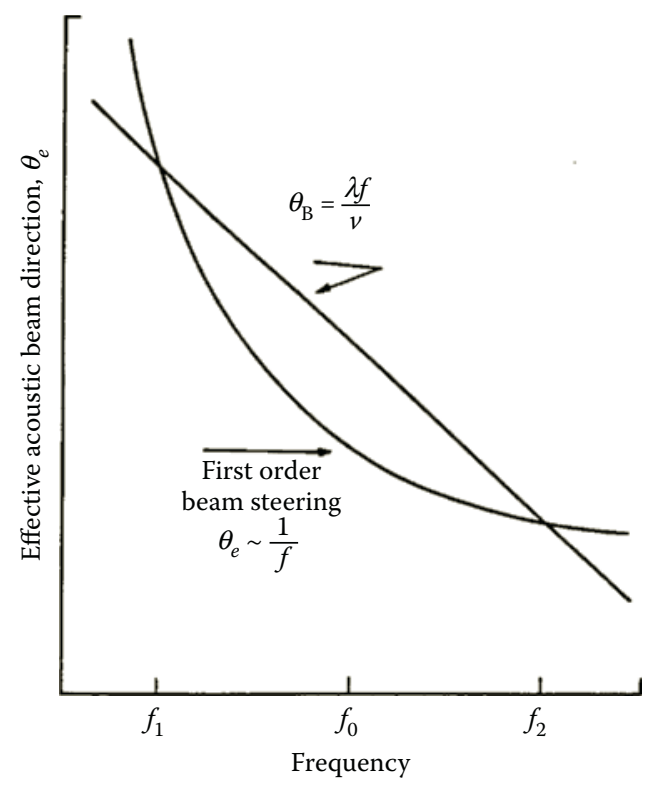

FIGURE 11.29

First-order beam steering with exact match at two frequencies.

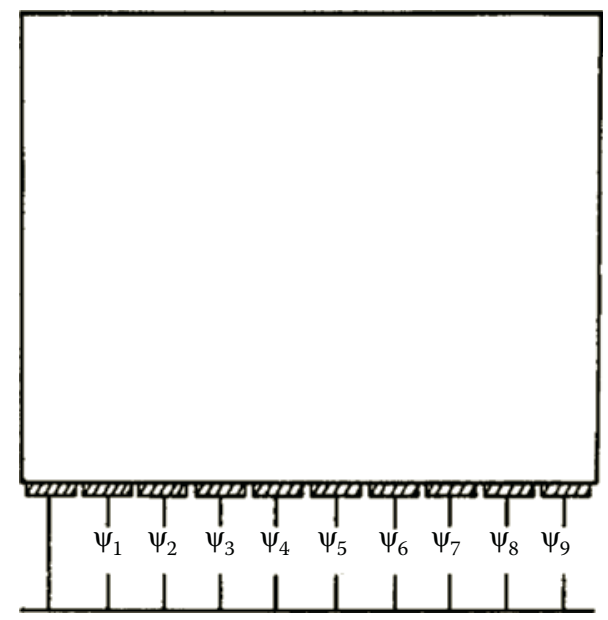

FIGURE 11.30

Ten-element phased array transducer, in which $\Psi=0,90,180$, or $270^{\circ}$, leading to diffracted intensity less than 0.8 $\mathrm{dB}$ lower than for perfect beam steering.

For frequencies near the midband of any of the transducer elements, the incident light will interact strongly only with the sound wave emanating from that element; interaction will be weak from the other elements both because the angle of incidence will be mismatched and the frequency will be far from the resonance frequency of those elements.

On the other hand, for frequencies that are midway between the resonance frequencies of adjacent elements, that is, $\left(f_{01}+f_{02}\right) / 2$ or $\left(f_{02}+f_{03}\right) / 2$, the contributions to the acoustic fields from both elements are about equal and the effective wavefront direction lies 


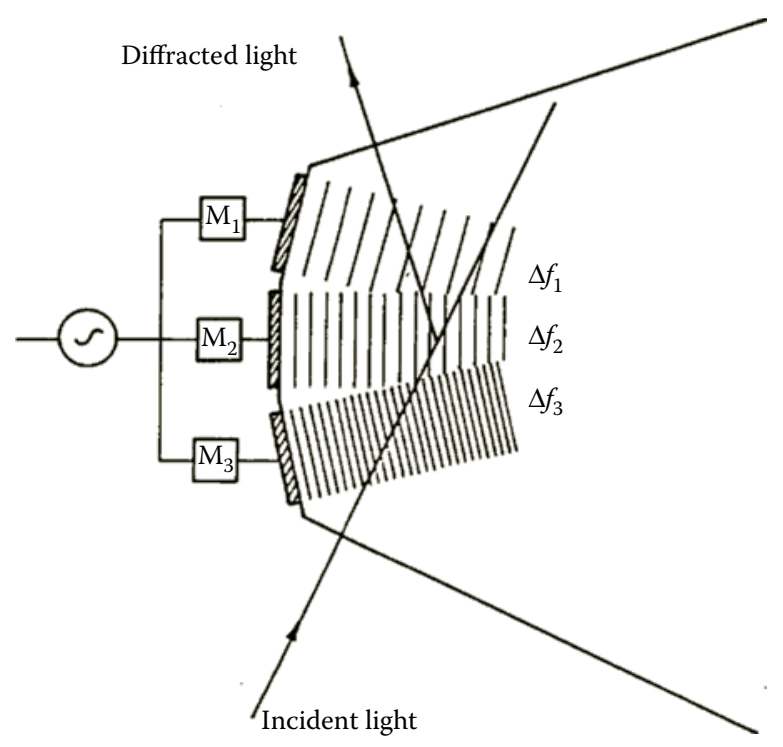

FIGURE 11.31

Tilted transducer array, in which each element is optimized for part of the entire frequency band.

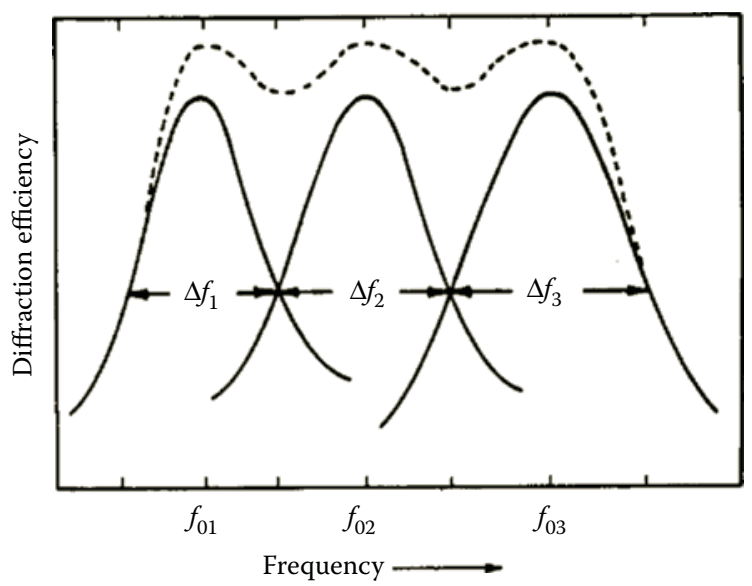

\section{FIGURE 11.32}

Diffraction efficiency of a three-element tilted transducer array. Solid curves represent efficiency of individual elements, and dotted curve represents efficiency of entire array.

midway between those of the components. Thus, the array behaves very much as if the acoustic wave were steering with frequency, although this is not true in a strict sense. The diffraction efficiency of the tilted transducer array is shown in Figure 11.32, in which the solid curves represent the efficiency of the individual elements and the dotted curve represents the overall efficiency. There will typically be about $1 \mathrm{~dB}$ of ripple across the full band, which is acceptable for most applications.

There are two additional advantages to the tilted array transducer. First, it is obviously relatively simpler to design a larger overall acoustic bandwidth, since each element of the tilted array need be only about one-third of the total bandwidth. Second, tilted-array 
transducers can generally be operated more deeply in the Bragg mode, as the combined acoustic wavefronts from adjacent elements are twice the length of that from a single element. If the second-order diffracted light is sufficiently low, then operation over a frequency range larger than one octave is possible. The elements of the array can be connected in parallel since they will tend to behave as bandpass filters, the power being directed to the element with the closest frequency range. In practice, it is generally necessary to provide impedance-matching networks, as indicated in Figure 11.31, because of the low reactance obtained with a parallel network.

\subsection{ACOUSTO-OPTIC DEVICE FABRICATION}

\subsubsection{Cell Fabrication}

The AO material is in most cases a crystal or an optical-grade glass. For crystalline materials, specific acoustic and optic propagation axes are typically required and the crystallographic orientation of the material must therefore be identified and marked. A typical cell configuration is shown in Figure 11.33. The optical window surfaces are prepared on the faces perpendicular to the optical axis.

These surfaces are typically polished to a high-quality window surface with a flatness of $\lambda / 20$ or better. Because the cells may be relatively thick (up to several centimeters) the homogeneity of the optical material may be a significant factor in the overall wavefront distortion. Therefore, the wavefront distortion may need to be specified for the cell in transmission.

Optical scatter is often a critical parameter for AO devices used in scanning systems. A portion of scatter from the zero-order beam will fall within the aperture of the deflected beam. This scattered light is unmodulated and may limit the extinction ratio in AOM applications. Scatter may occur at the surfaces due to contamination or imperfections or within the material due to defects or inhomogeneities.

Unlike most lenses and mirrors where the clear aperture is in the middle of the optic, the desired clear aperture of an AO device usually starts at the edge of the transducer face. For AOMs, the beams may be centered less than $0.5 \mathrm{~mm}$ from the edge of the cell. Therefore,

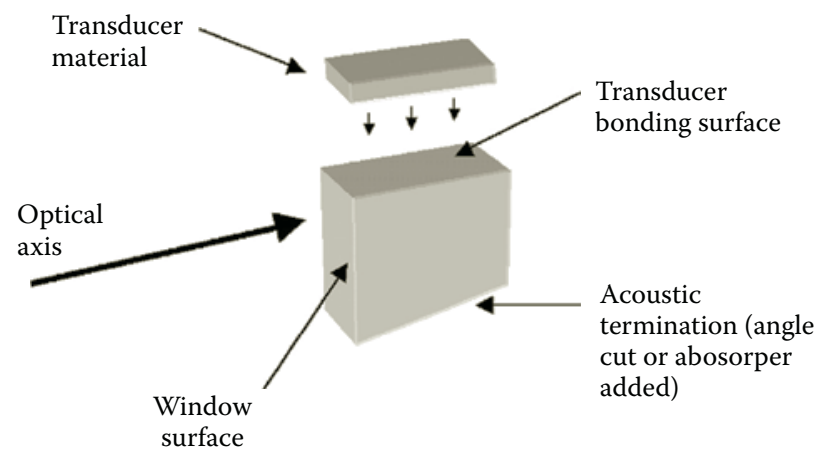

FIGURE 11.33

Bonding of acoustic transducer and acousto-optic medium. 
special care must be taken to achieve the surface flatness, polish, and antireflection coating required across the entire clear aperture.

In particular with AOMs, the laser beam is focused to a small spot at the AOM to achieve the desired rise time and can lead to high optical intensity. Therefore, antireflection coatings often must be specified with high damage thresholds.

The acoustic transducer bonding surface is nominally parallel to the optic axis. This surface is prepared for bonding typically by polishing to an optical-quality surface. In most applications, the face of the cell opposite of the acoustic transducer surface is cut or ground at an off angle so that acoustic waves will not reflect directly back to the optical aperture or acoustic transducer. This is to avoid modulation or intermodulation from acoustic echo. Other techniques employed to reduce back reflection are to bond an acoustic absorbing material to back surface or grinding a rough finish on the back surface to diffuse the back reflection.

\subsubsection{Transducer Bonding}

For transducers in the frequency range for which crystal plates are bonded to the delay medium, the bonding procedure is probably the most critical and most difficult step in fabricating the structure. The bonding layer can drastically modify the transmission of acoustic energy between the piezoelectric and the delay media; this is because the bond layer must provide molecular contact between the two surfaces, which will otherwise result in incomplete transfer, and because the mechanical impedance of the bond layer may produce a large acoustic mismatch with low transmission. In addition to these considerations, if the bond material is acoustically lossy, further decrease in transmission will result.

Because of the special properties required, there is only a very limited number of known bonding materials available. For temporary attachments, a commonly used agent is "salol," phenyl salicylate. It is easily applied as a liquid, which is crystallized by addition of a small seed. It is reliquified by gentle heating, and therefore is useful for various test measurements, but does not yield wide bandwidth or efficient coupling. A more satisfactory bond is made with epoxy resin, mixed to a very low viscosity, which may be compressed to a layer less than $1 \mu \mathrm{m}$ thick before setting. Such thin layers require a high degree of cleanliness to avoid inclusion of any dust particles. Because of the low impedance of epoxy compared with such transducer materials as lithium niobate, thicker bonding layers would cause serious impedance mismatch problems around $100 \mathrm{MHz}$, where this technique has been successfully used.

Good results can also be obtained with a low-viscosity, ultraviolet, light-cured cement. For frequencies higher than about $100 \mathrm{MHz}$, other techniques, capable of yielding still thinner bond layers, which must be kept to a small fraction of an acoustic wavelength, must be used. Vacuum-deposited metallic layers are well suited for this purpose, because their thickness can be very accurately controlled down to the smallest dimensions, and impedances much closer to those of commonly used piezoelectric materials are available. Very good results were first obtained with indium bonds, ${ }^{49}$ which are deposited to a thickness of several thousand angstroms on both surfaces, and without removal from the vacuum systems are mated under a pressure of about 100 psi. This technique yields a cold-welded bond, which has excellent mechanical properties with large acoustic bandwidth, if properly designed, and low insertion loss at frequencies of hundreds of megahertz.

The greatest fabrication difficulty is due to the necessity of maintaining the deposited films under vacuum to prevent oxidation. This requires a vacuum system with rather elaborate fixtures to bring the two surfaces together after film deposition and to apply the 
hydraulic pressure. The inside of a vacuum system in which this procedure is carried out is shown in Figure 11.34. The substrates are held on either side of the evaporation filament sources during film deposition and are quickly brought into contact before contamination can occur. Using this technique, compression bonds of indium, tin, aluminum, gold, and silver are routinely made. It is essential for these procedures to be carried out in a dustfree atmosphere in order to avoid contaminating the interface with particulates. Even the smallest particles will prevent good acoustic contact between the transducer and the cell, so that fabrication must be carried out in a clean-room facility. A typical AO device clean room is shown in Figure 11.35.

In a modification of the indium compression bond, ${ }^{50}$ which allows the freshly deposited indium surfaces to be removed from the vacuum system for handling, the work is then placed in an oven under a pressure of several hundred psi, raised in temperature to slightly below the melting point of indium $\left(156{ }^{\circ} \mathrm{C}\right)$, and slowly cooled. This procedure forms a molecular bond in spite of the oxidation that may occur, and gives results similar to the vacuum bond. The principal drawback is that upon cooling, differential thermal expansion coefficients between the delay line material and the transducer material may set up unacceptable strains in the optical path. For some systems, this may not be a problem; for example, quartz or even lithium niobate transducers on fused or crystal quartz delay lines can be routinely made by this method. On the other hand, such crystals as tellurium dioxide require a great deal of care in handling, since they are extremely sensitive to thermal shock and strains. Differential contraction between the crystal and transducer for a bond made in this fashion may easily be severe enough to fracture the crystal. Therefore, its applicability will depend upon the materials and sizes involved and upon the degree of freedom from residual strain required.

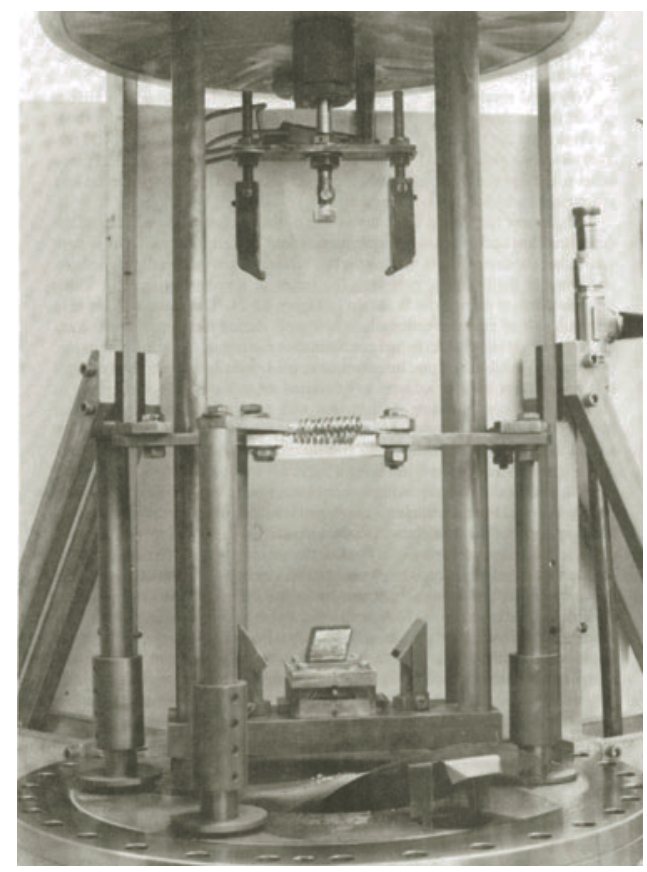

FIGURE 11.34

Vacuum compression bonding system. Metal films are deposited on transducer and delay line surfaces, which are then brought into contact. 


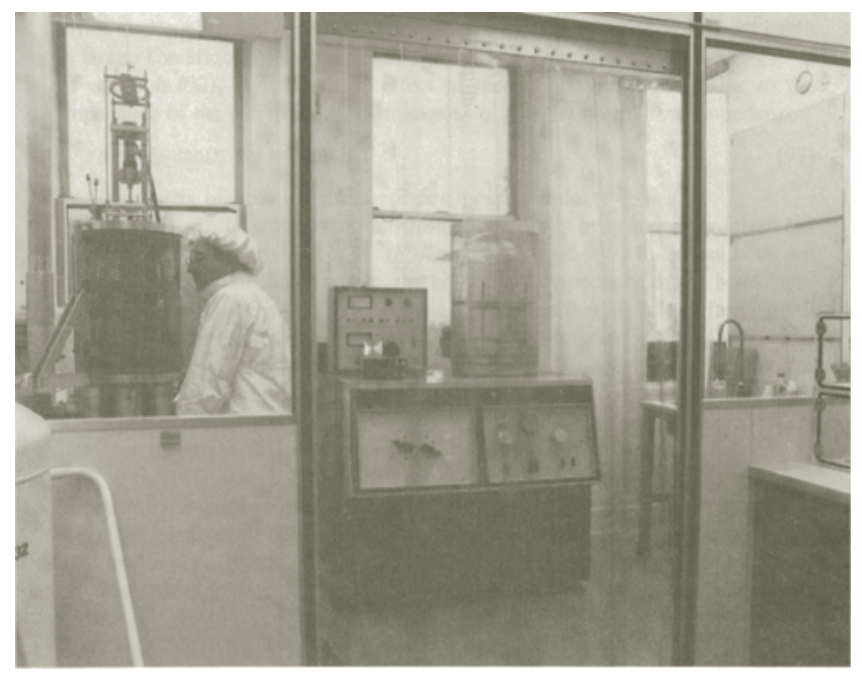

FIGURE 11.35

A clean room for acousto-optic device fabrication.

For frequencies approaching $1 \mathrm{GHz}$, the attenuation of indium layers may become excessive, and better results can be achieved with metals with lower acoustic loss constants. Among such metals are gold, silver, and aluminum.

Although these are made by the vacuum compression method, they generally require higher pressure. Still another method that has been used with these, as well as indium, is ultrasonic welding. ${ }^{51}$ The chief advantage to be gained is that the procedure is carried out in normal atmosphere, since the ultrasonic energy breaks up the oxidation layer that forms on the surface. Some heating occurs as a result, but the temperature remains well below that required in the indium thermocompression method, with much lower residual strains. The technique requires the simultaneous application of pressures up to $3000 \mathrm{psi}$; this may be excessive for easily fractured or deformed materials or where odd-shaped samples are involved. A summary of the important properties of a few bonding materials, also used for electrodes and intermediate impedance-matching layers, is given in Table 11.6.

At lower frequencies, the effects of thin electrode and bonding layers on the performance of the transducer may be entirely negligible, but near $100 \mathrm{MHz}$, they become increasingly large, and even for layers less than $1 \mu \mathrm{m}$ thick the effect may not be negligible if the impedance mismatch to the rest of the structure is large. The effects of the electrode layer can be determined by setting $Z_{b}=0$ in Equation 11.107, and the entire effect of the back layers will be due to the impedance of the electrode $z_{b l}$ of thickness $t_{b t}$, so the normalized impedance

$$
z_{b}=j z_{b l} \tan \left(t_{b l} g\right)=j \tan d
$$

and the matrix of Equation 11.107 becomes more complex.

The effect of the bond layer and front electrode is even more complex, but an interesting illustrative example of varying the bond layer thickness is shown in Figure 11.36. For this example, the normalized impedance of the bond layer is taken to be rather low, $z=0.1$, and it can be seen that even for a fairly small thickness, the effect on the transducer loss is 
TABLE 11.6

Acoustic Properties of Bond Layer Materials

\begin{tabular}{|c|c|c|c|c|c|c|}
\hline \multirow[b]{2}{*}{ Material } & \multicolumn{3}{|c|}{ Longitudinal waves } & \multicolumn{3}{|c|}{ Shear waves } \\
\hline & $\begin{array}{l}\text { Velocity } \\
(\mathrm{cm} / \mathrm{s})\end{array}$ & $\begin{array}{c}\text { Impedance } \\
\left(\mathrm{g} / \mathrm{s} \mathrm{cm}^{2}\right)\end{array}$ & 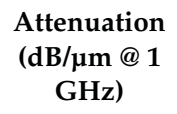 & $\begin{array}{l}\text { Velocity } \\
(\mathrm{cm} / \mathrm{s})\end{array}$ & $\begin{array}{l}\text { Impedance } \\
\left(\mathrm{g} / \mathrm{s} \mathrm{cm}^{2}\right)\end{array}$ & 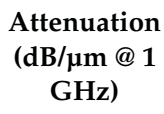 \\
\hline Epoxy & $2.6 \times 10^{5}$ & $2.86 \times 10^{5}$ & Very large & $1.22 \times 10^{5}$ & $1.34 \times 10^{5}$ & Very large \\
\hline Indium & $2.25 \times 10^{5}$ & $16.4 \times 10^{5}$ & 8 & $0.19 \times 10^{5}$ & $6.4 \times 10^{5}$ & 16 \\
\hline Gold & $3.24 \times 10^{5}$ & $62.5 \times 10^{5}$ & 0.02 & $1.2 \times 10^{5}$ & 23.2 & 0.1 \\
\hline Silver & $5.65 \times 10^{5}$ & $38 \times 10^{5}$ & 0.025 & $1.61 \times 10^{5}$ & $16.7 \times 10^{5}$ & \\
\hline Aluminum & $6.42 \times 10^{5}$ & $17.3 \times 10^{5}$ & 0.02 & $3.04 \times 10^{5}$ & $8.2 \times 10^{5}$ & \\
\hline Copper & $5.01 \times 10^{5}$ & $40.6 \times 10^{5}$ & & $2.11 \times 10^{5}$ & $18.3 \times 10^{5}$ & \\
\hline
\end{tabular}

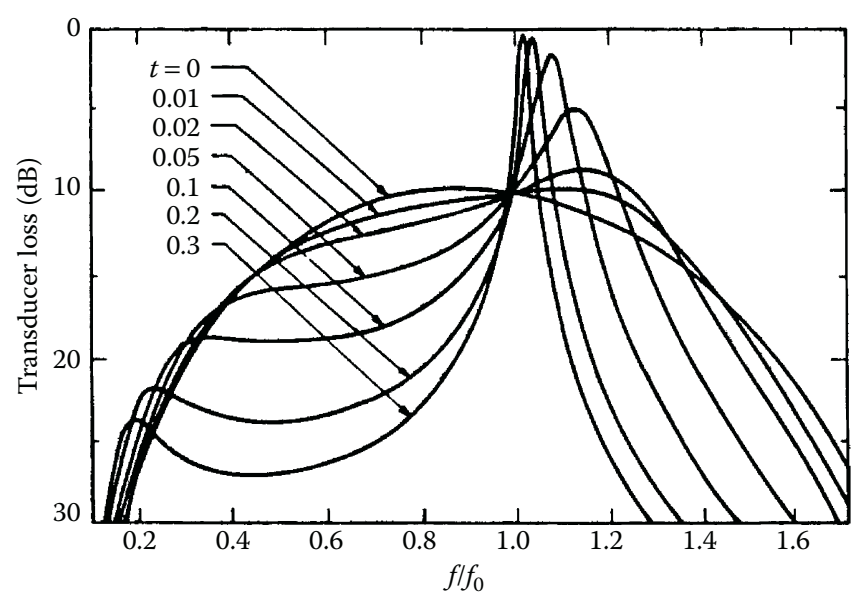

FIGURE 11.36

Transducer loss for various values of normalized transducer thickness $t$ and intermediate layer normalized thickness 0.1. $R_{s}=\left(\omega_{0} C_{0}\right)^{-1}, z_{0 t}=1, k=0.2$.

quite marked. Such a low value of impedance would correspond to the nonmetallic bond materials, but for the metallic bond materials the impedance mismatch would not be as severe, and the curve of transducer loss would be correspondingly less influenced. This influence of intermediate layers on the shape of the transducer loss curve can be used to determine the bandpass characteristics of the transducer structure. Such impedance transformers can be used, for example, to make the response symmetric about the band center $f_{0}$ by making the intermediate layer thickness one-quarter wavelength at $f_{0}$. By choosing other values for the thickness, the bandwidth can be enlarged, ripples smoothed, or various distortions introduced. In general, however, any such objectives are achieved at the expense of increased transducer loss.

\subsubsection{Packaging}

$\mathrm{AO}$ device packaging must take into account needs for optical mounting and electrical connectivity and in many cases, thermal path management as well. Often it is desirable to attach the optical cell to a metal mount suitable for mechanical attachment in a larger 
optical system. This is achieved by bonding one or more of the surfaces to the mount with adhesive. Low shear strength adhesives are needed in many cases to alleviate temperature-induced strain between the metal and the optical material. This is because most metals have a thermal expansion coefficient significantly greater than that of glass or other optical crystals. For example, the linear expansion coefficient of aluminum is approximately 23 parts per million per ${ }^{\circ} \mathrm{C}$ compared to only 0.5 parts per million per ${ }^{\circ} \mathrm{C}$ for fused silica. If the cell is bonded with a thin layer of high strength epoxy, difference in expansion over temperature may cause significant strain birefringence in the optical cell.

For devices that use a watt or more of RF power, thermal management becomes important. Most of the power input to the device will end up as heat either from electrical resistive losses or acoustic attenuation loss. This heat must be transferred from the modulator efficiently enough to keep the operating temperature within desired limits. A significant fraction of the input power may be lost at the transducer surface of the AOM, including resistive losses from bond wires and electrodes, and acoustic losses in the transducer and bond layers. Localized heating from the transducer will form temperature gradients in the optical cell that can cause optical distortion due to the change in index with temperature. The effect may be reduced by attaching a heat sink to the back side of the transducer or by choosing an optical aperture further from the transducer. The remaining power is converted into acoustic power that dissipates inside the cell. Because most optic materials are poor thermal conductors, the cell may need to be intimately contacted with a good heat sink path on as much surface area as possible to keep temperature rise to a minimum.

For modulators operating with frequencies of hundreds of $\mathrm{MHz}$ or more, the transducer electrode is typically deposited as a thin film (less than $1 \mu \mathrm{m}$ thickness) by vacuum deposition. Electrical connection to the electrodes is made by bond wiring. Direct soldering to the electrodes may cause damage to the electrode and cause excess loading on the acoustic transducer. A circuit card may be used to bring the input RF from an external connector up to the acoustic transducer. The feed circuit typically includes a passive matching circuit to optimize the impedance matching, nominally $50 \Omega$.

\subsection{APPLICATIONS OF ACOUSTO-OPTIC SCANNERS}

\subsubsection{Multichannel Acousto-Optic Modulator for Polygonal Scanner}

AOM or AOD can be fabricated with many independent channels on a single monolithic device. This approach allows multiple parallel beams to be modulated with a single device. This approach is used in the Etec Systems ALTA 3000 mask writing machine, which writes semiconductor photomasks using a rasterized simultaneous 32-beam scan.

The scanner architecture of the ALTA 3000 system is illustrated in Figure 11.37. An argonion laser generates a single Gaussian beam at a wavelength of approximately $364 \mathrm{~nm}$. A beam-splitter subassembly creates 32 separate beams, referred to as the brush, that pass through the AOM, which can independently turn on and off each of the beams. The modulation of the beams is controlled by the data path subsystem by varying the RF power to each channel of the AOM. The scan is created by a rotating polygonal mirror, and the scanned angle is converted to a spatial displacement by an $f-\theta$ lens. The final image of the brush at the photomask is obtained after transmitting it through a 20×, $0.6 \mathrm{NA}$ reduction lens. At the image plane, the FWHM diameter of the spot size is approximately $360 \mathrm{~nm}$. 


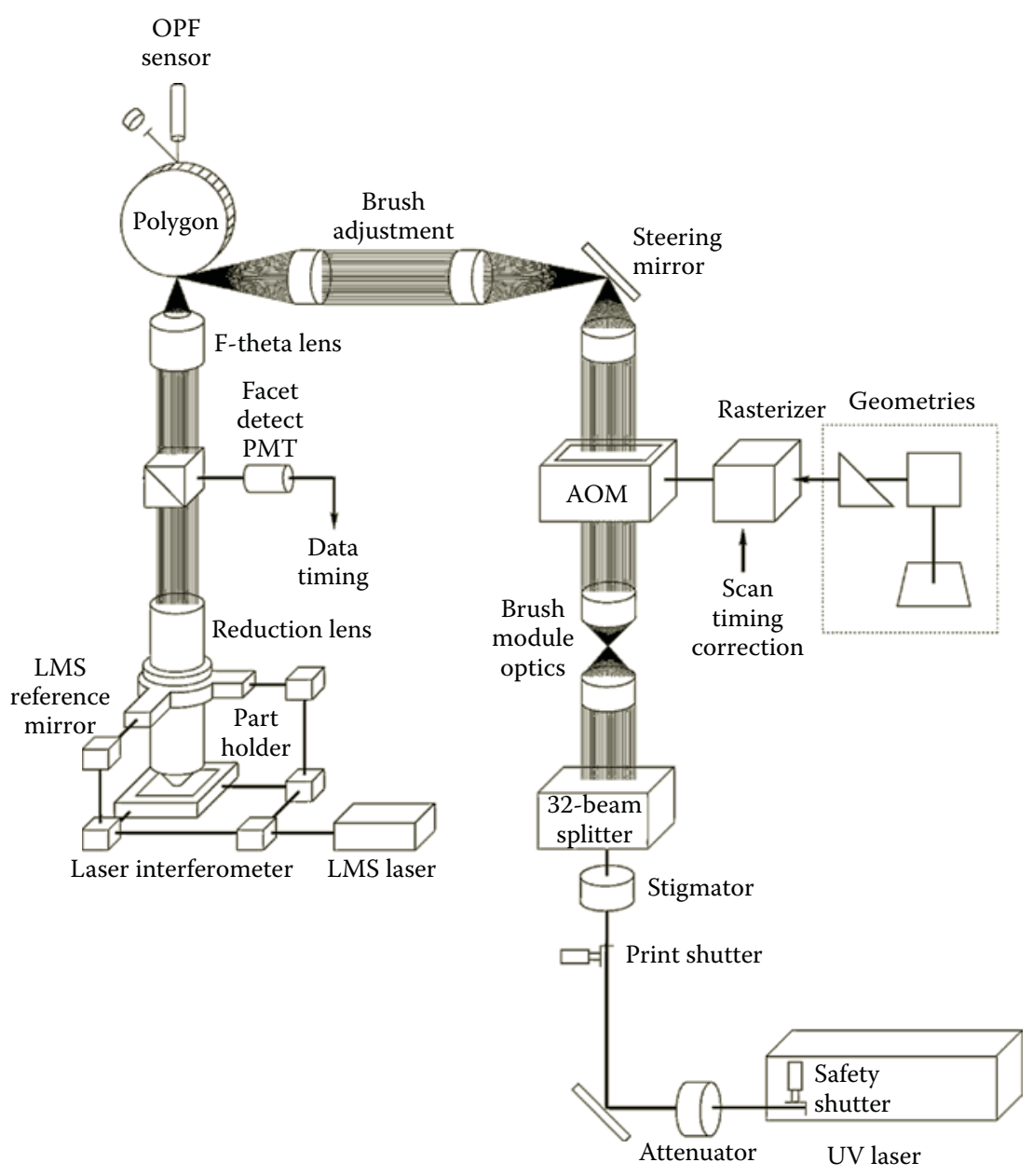

FIGURE 11.37

Use of a multichannel modulator in a precision semiconductor mask writing machine.

During the scan, a translation stage moves the photomask perpendicular to the direction of scan.

The AOM and other optics are made from UV-grade fused silica, which has excellent transmittance and resistance to radiation darkening at UV wavelengths. Unfortunately, the $M_{2}$ value for fused silica is relatively low, making power requirements for the modulator an important design parameter. A nominal drive level of $500 \mathrm{~mW}$ per channel at 200 $\mathrm{MHz}$ is required to achieve a diffraction efficiency of $50 \%$.

The brush used to print at the photomask is an image of the one first created at the AOM. Therefore, the arrangement of beams at the AOM is dictated by the requirements for the final print. In this case, this arrangement is two sets of 16 beams with each beam separated by approximately $31 / e^{2}$ beam diameters. The size of the beams at the AOM is set at 144 $\mu \mathrm{m} 1 / e^{2}$ diameter based on the modulation bandwidth requirement of 50 megapixels per second, and the beam-to-beam spacing is $412 \mu \mathrm{m}$.

The design of the transducer electrode size and shape must consider the impacts on power efficiency, beam distortion, and channel-to-channel cross-talk. The limited aperture 
width and angular acceptance window of this design distort the output beam to a net farfield ellipticity of approximately 1.3:1, which is acceptable for this application. The modulator also introduces astigmatism, which would be detrimental to the system performance. This problem is corrected by precompensating optics referred to as the stigmator on Figure 11.37.

The tight channel spacing requires careful attention to the prevention of electrical cross talk in the feed network to the transducer electrodes. Radio frequency signals are fed to the modulator from the system electronics by an array of coaxial cables. The coaxial cables are connected to a printed circuit card with an individual trace for each of the 32 channels. Each trace ends with a land registering to one of the transducer electrodes. Bond wires are applied to make the final connection between the feed circuit and transducer electrodes.

When all 32 channels are on, this equates to $16 \mathrm{~W}$ of RF power. This creates localized heating in the AO cell near the transducer, also corresponding to the useful optical aperture of the AOM. Because the index of the fused silica is temperature dependent, these localized temperature gradients correspond to index gradients in the glass that may cause several waves of distortion across the optical aperture. A temperature gradient across the aperture will have the effect of an optical wedge, causing the angular orientation of the beams to shift. To minimize these effects, a heat sink is applied to the back side of the transducer to keep the temperature rise at the transducer as small as possible. The AOM mount is also water cooled to carry the bulk heat away from the device and the surrounding optical system.

\subsubsection{Infrared Laser Scanning}

AO beam scanners for use with IR lasers have been under consideration in recent years by the aerospace industry in connection with laser radar and optical communications systems. Where system requirements place excessive demands on mechanical scanning methods, various electronic approaches become attractive. In general, the carbon dioxide laser, with wavelengths from 9 to $11 \mu \mathrm{m}$, is the most common one for long-wavelength operation. There are a number of electronic approaches to IR beam scanning besides AO, and all of them are quite difficult to implement, usually for reasons related to the long interaction length needed to achieve large optical phase excursion. For AO diffraction, we have seen that the RF power needed for a given diffraction efficiency increases quadratically with wavelength. At $10.6 \mu \mathrm{m}$ therefore, 280 times the power for $0.633 \mu \mathrm{m}$ is necessary. Clearly, there will be severe constraints on the available materials for such devices, and on their performance. Referring to Table 11.4, we can see that only a few materials that transmit to $11 \mu \mathrm{m}$ and have large AO figure of merit have been identified. The most common of these is germanium, which can be purchased in very large single crystals of excellent optical quality; germanium AO scanners have been commercially available for a number of years. They operate best in the isotropic mode, typically near $100 \mathrm{MHz}$ RF. Another favorable IR material for use in the 9 to $11 \mu \mathrm{m}$ range is thallium arsenic selenide, which has very recently become available on a commercial basis. This crystal is best used in an anisotropic mode, as described in a previous section. The AO figure of merit is very high due to the low value of shear acoustic wave velocity. The low velocity produces another result that may simplify the design of scanner optics. The scan angles at IR wavelengths are quite large; the angular dispersion for 10.6- $\mu \mathrm{m}$ carbon dioxide wavelength for this Bragg cell is shown in Figure 11.38. For an RF bandwidth of 30\% around $110 \mathrm{MHz}$ center frequency, a scan-angle range of $16^{\circ}$ is reached. For many applications, no magnification of the scan angle will be needed, as may be the case for $\mathrm{AO}$ scanners in the visible. 


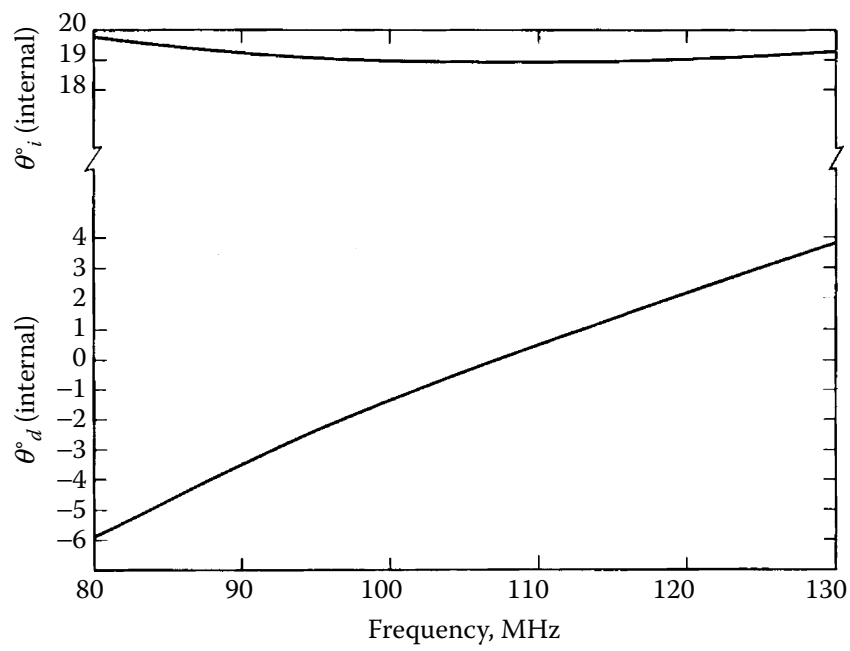

FIGURE 11.38

Anisotropic Bragg diffraction in TAS at $\lambda=10.6 \mu \mathrm{m}$.

One of the major problems associated with carbon dioxide laser beam scanners is heating, due both to absorption of optical energy and RF power heating. If very high laser beam powers are used, then even a small absorption coefficient in the scanner may cause unacceptable heating. For germanium and thallium arsenic selenide, the absorption coefficients at $10.6 \mu \mathrm{m}$ are 0.032 and $0.015 \mathrm{~cm}^{-1}$, respectively. The thermal conductivity of germanium is much higher than that of thallium arsenic selenide, but design considerations may favor one or the other, depending upon the detailed effects of thermal gradients. High RF power operation is limited by heating at the transducer, which will eventually damage the transducer bond. Such thermal effects can be reduced by water or air cooling the RF mount and by heat sinking the transducer; a photograph of a high-power transducer with a matching sapphire heat sink, which also serves to make electrical contact, is shown in Figure 11.39. The resolution of such IR AO scanners will, in general, be limited by RF heating if high diffraction efficiency is to be obtained. This comes about because a large interaction bandwidth requires a small interaction length, so that high efficiency can only be achieved by high power. The relationship between resolution and acoustic power for the IR scanner materials is shown in Figure 11.40. If CW operation is required, it can be seen that no more than a few hundred spots can be obtained for an aperture of 1 or $2 \mathrm{~cm}$.

\subsubsection{Two-Stage Acousto-Optic Scanner}

AO deflection has been successfully applied to semiconductor photomask inspection. The KLA-Tencor 3000 series mask inspection machine checks photomasks for defects by scanning a focused spot across the mask and detecting the transmitted light (brightfield inspection). In this application, the feature sizes are very small $(0.3 \mu \mathrm{m})$ compared to the size of the mask to be inspected (typically 6 in diameter). To achieve desired throughput, a net scan rate of 50 megapixels per second is required. The number of spots is too great to cover the photomask with a single scan, therefore the approach is to use a high-speed optical scanner to produce a lineal subscan, and use $x-y$ mechanical translation to pass the photomask beneath the scan location. Significant control is required to provide the precise 


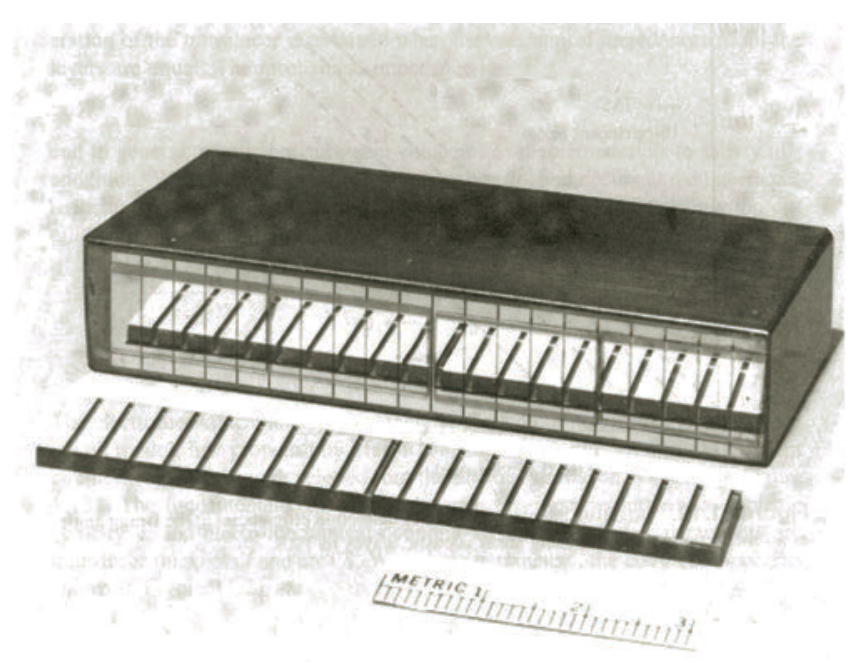

FIGURE 11.39

Transducer structure on TAS acousto-optic device, with matching heat sink for high RF power operation.

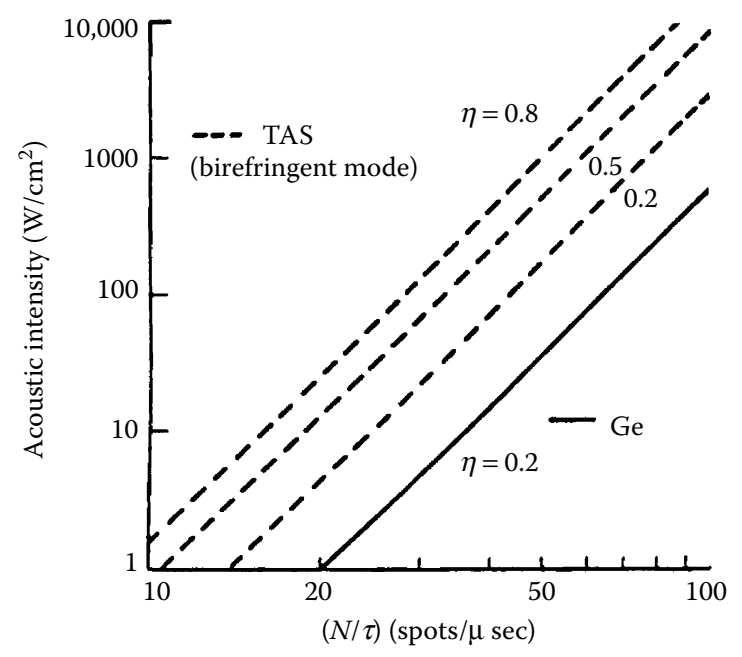

FIGURE 11.40

Acoustic intensity for various deflection efficiencies at the Bragg angle as a function of bandwidth using shear acoustic waves in TAS.

translation required and register all the subscan data together such that the data for the entire photomask can be seamlessly reconstructed.

\subsubsection{Scanner Optics}

The lineal scan is produced by an AO scan pair and has no moving parts. A block diagram of the scan optical portion of the system is shown in Figure 11.41. The source for the scan beam is an argon-ion laser operating at 488-nm wavelength. The beam is passed through a spatial filter and then focused to a $400-\mu \mathrm{m}$ beam diameter at the first $\mathrm{AO}$ device, the 


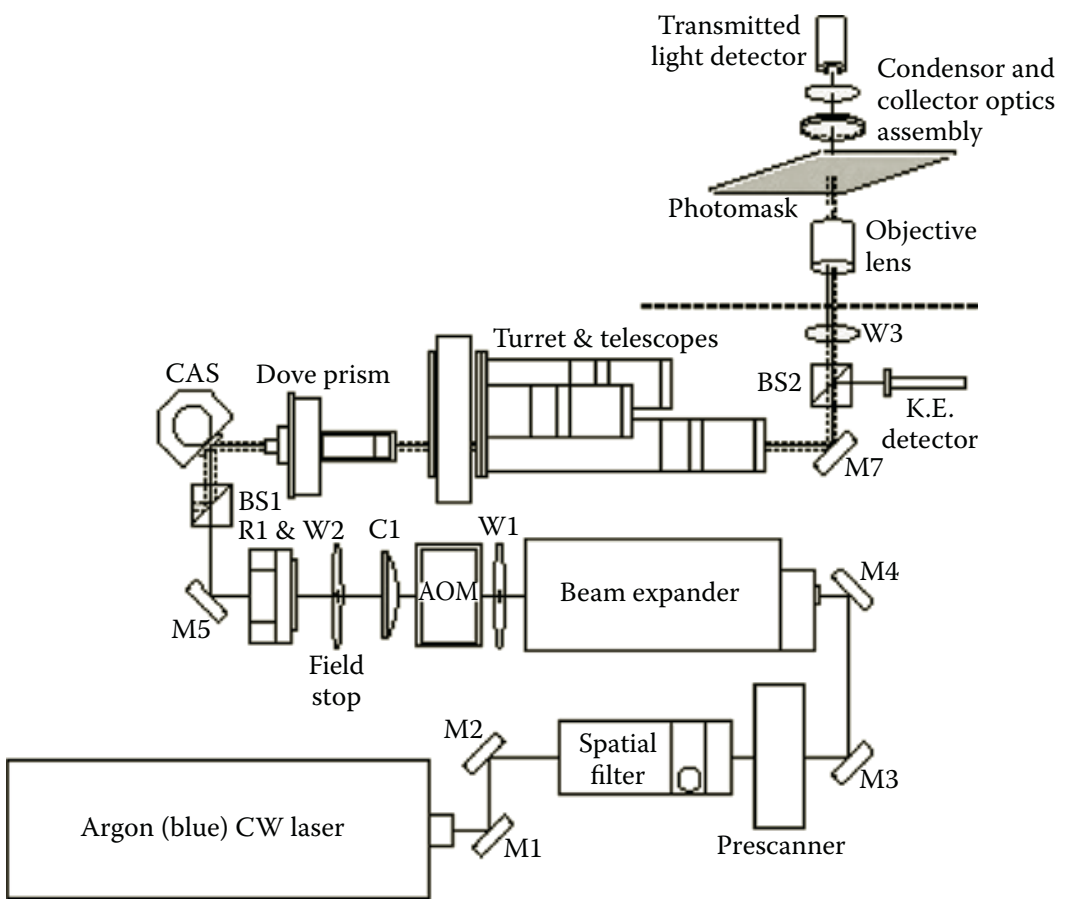

\section{FIGURE 11.41}

Use of an acousto-optic deflector (prescanner) and traveling chirp lens (AOM) in a semiconductor mask inspection machine.

predeflector. This device is made from SF-6 glass and operates in the longitudinal acoustic mode. The center frequency for the device is $90 \mathrm{MHz}$, and the operating bandwidth is 14.4 $\mathrm{MHz}$. This corresponds to a deflection angle in air of 12.5 mrads \pm 1 mrads (Figure 11.42).

Following the predeflector, prescan optics transform the deflector output into a telecentric scan with magnification appropriate for the traveling chirp lens. The spot size at the input of the traveling chirp device is $12 \mathrm{~mm} 1 / e^{2}$, and is designed to overfill the traveling chirp lens.

The chirp lens is produced from $\mathrm{TeO}_{2}$, and uses the slow shear acoustic mode. Unlike the predeflector, the diffraction performance of a slow shear $\mathrm{TeO}_{2}$ is very polarization dependent, and requires the input to be right circularly polarized. A wave plate is used to convert the linear polarization from the laser to circular polarization ahead of the $\mathrm{TeO}_{2}$ device. The lens is formed from a linear chirp from 75 to $125 \mathrm{MHz}$ over $7.5 \mu \mathrm{s}$. This makes the lens aperture in the scan direction $4.6 \mathrm{~mm}(7.5 \times 0.616)$.

The aperture size in the cross-scan direction is controlled by the acoustic transducer height and is set to also be approximately $4.6 \mathrm{~mm}$. The length of the scan is $14 \mu \mathrm{s}$ or 8.6 $\mathrm{mm}$. Therefore, the traveling chirp cell must have a clear aperture of at least $12.2 \mathrm{~mm}$ in the scan direction.

A cylinder lens is placed immediately after the chirp device to focus the spot on the cross-scan axis. A scan plane occurs at one focal length from the chirp lens. This is the object plane that is relayed and demagnified to produce the final scan at the photomask. Using the approximation $N=\tau \Delta f$, the number of resolvable spots from the predeflector is 1.6. While this performance would be of little use in direct scanning applications, its purpose in this application is to track the traveling chirp lens and maintain optimal illumination. 


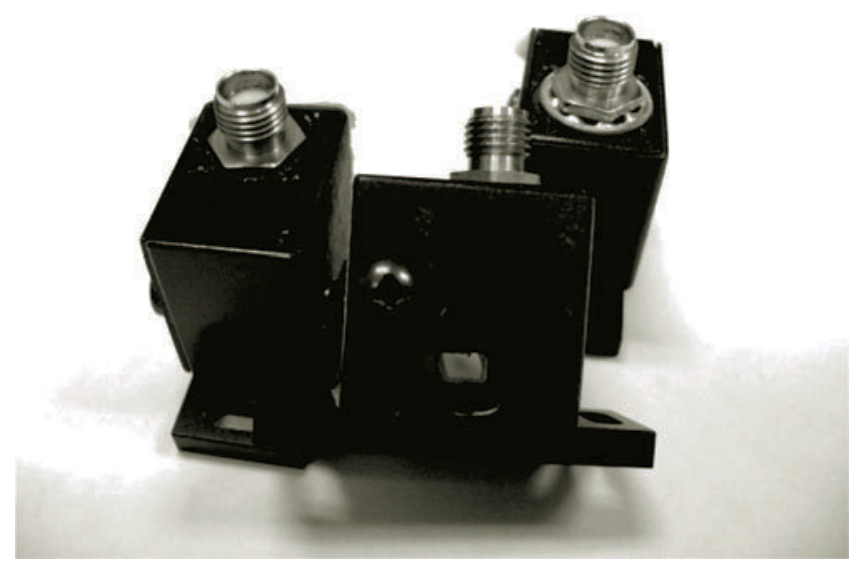

FIGURE 11.42

A typical 100-MHz-bandwidth acousto-optic modulator (courtesy MVM Electronics, Inc.).

The spot gain from the traveling chirp, also estimated from the time-bandwidth product, is 375 . Therefore, the scan resolution is dominated by performance of the traveling chirp lens. The approximate scan size based on these approximations is 600 spots. However, due to limited aperture at the traveling chirp lens, the scanned spot is better approximated by an Airy disk function than a Gaussian, and the scan size based on the null-to-null spacing approximately 1000 spots.

\subsubsection{Driver}

Both AO devices are driven by analog electronics that consist of a voltage-controlled oscillator followed by amplifier stages. Linear voltage ramps are generated by the system electronics and supplied to the drivers to produce the linear frequency chirps required by the $\mathrm{AO}$ devices. Both drive inputs are derived from the same clock to ensure synchronization is maintained between the two devices. Note that chirp linearity is not critical for the predeflector as it would be with an $f-\theta$ configuration. In this system, the scan linearity is controlled by the propagation of the traveling lens and the dominant concern is variation of acoustic velocity in the traveling lens cell due to changes in temperature. Chirp linearity is critical for the traveling lens, as the nonlinearities in the chirp signal will appear as aberrations in the lens. Therefore, precompensation is included in the voltage ramp fed to the traveling chirp voltage-controlled oscillator to correct for inherent nonlinearities.

\subsubsection{Applications of Acousto-Optic Devices and Acousto-Optic Tunable Filters}

$\mathrm{AO}$ devices are used for a variety of applications and, depending on the area of specialization, comprise the following: AOM, AOD, frequency shifters (AOFS), tunable filters (AOTF), wavelength selectors (AOWS), and polychromatic modulators (PCAOM). Combining one of these $\mathrm{AO}$ devices with a mechanical scanner can produce scanning systems for laser printing, laser machining and engraving, wavelength-programmable scanning, lasik, medical and cosmetic laser systems, and many more. Here we will describe the above mentioned devices to provide insight into their limitations and typical product configurations. 
The AO effect is used in a variety of ways for controlling light. From the widely utilized modulation of laser beams, solid-state light scanning, and frequency shifting, to tunable filters and polychromatic light modulation. The basic scheme for all these devices involves the selection of proper $\mathrm{AO}$ materials and the optimization of the piezoelectric transducer to produce acoustic waves with efficient conversion of electrical power to ultrasonic power for the desired frequency range. Except in colinear tunable filters, all schemes utilize the propagation of acoustic and optical waves approximately at $90^{\circ}$ to each other. The theory of individual devices from the list above appears earlier in this chapter, this section serves to discuss the major aspects required to produce the desirable product in each category.

\subsubsection{Acousto-Optic Modulators}

The basic requirement of an AOM is the highest-speed or wide-bandwidth modulation of light with the highest optical throughput and list amount of electrical power. Limiting factors are the acoustic transit time and the limited fractional bandwidth of the piezoelectric transducer and the inefficient interaction of acoustic waves with optical waves. Optical transmission, damage range, and the acoustic attenuation of the material may become hard to overcome as we push the envelope of the design. The highest bandwidth achieved for an $\mathrm{AOM}$ is about $2 \mathrm{GHz}$ or $\sim 0.5 \mathrm{~ns}$ rise/fall time, with a top efficiency of less than $20 \%$. These parameters are considerably degraded as the optical wavelength increases toward IR wavelengths such as $10.6 \mu \mathrm{m}$. As a variation of a single AOM a linear array of transducers has produced an AOM device with up to 128 channels (Figure 11.43 through 11.46).

\subsubsection{Acousto-Optic Deflectors}

The function of an AOD is to move or scan optical beams electronically. The dependence of the optical diffraction angle on acoustic wavelength makes this possible. The desired parameters are the maximum number of resolvable spots, the highest optical throughput

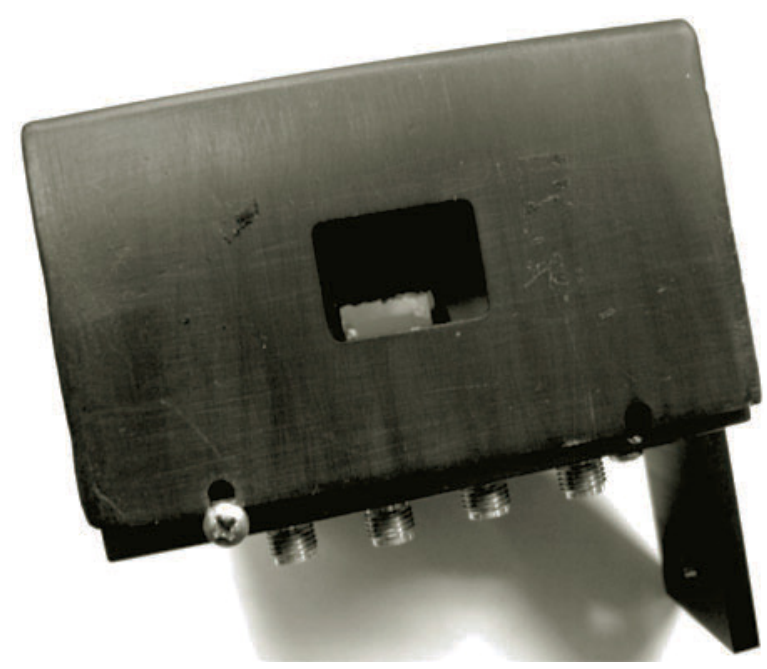

FIGURE 11.43

A typical 16-channel acousto-optic modulator (courtesy MVM Electronics, Inc.). 


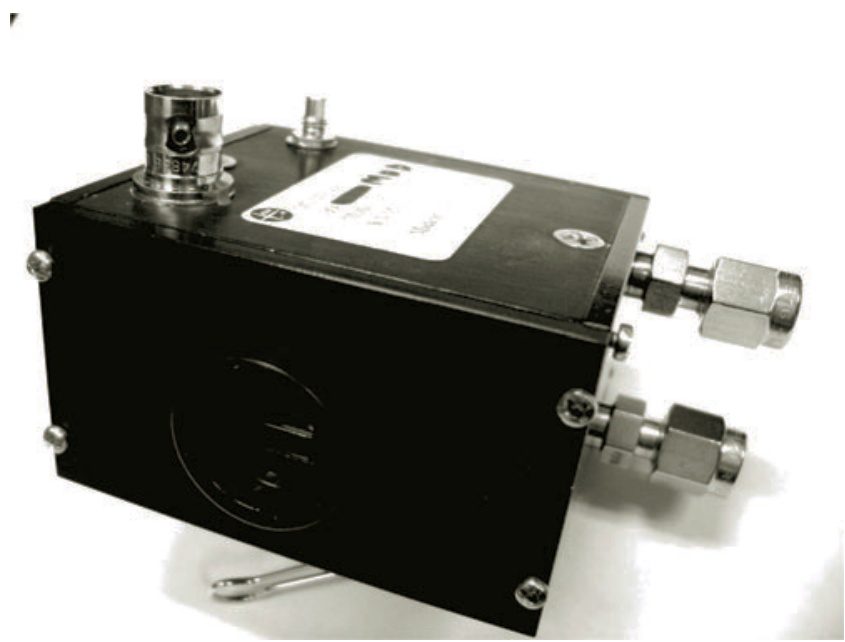

FIGURE 11.44

A typical Ge acousto-optic modulator for infrared modulation (courtesy MVM Electronics, Inc.).

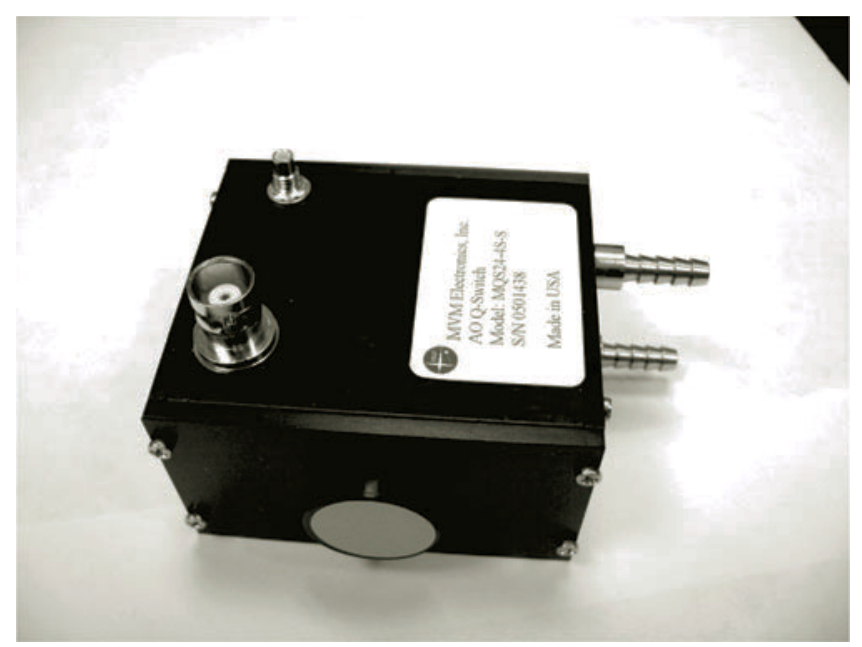

FIGURE 11.45

A typical quartz AOM for laser Q-switching application (courtesy MVM Electronics, Inc.).

and the list electrical power. Here, the transducer bandwidth and acoustic attenuation of the material place severe limits on the achievable results. Speed of scanning and uniformity of optical throughput further reduce the performance. The maximum resolvable spots achieved are about 2000, with a scan time of about $10 \mu$ s or larger. Most AO materials have identical AO properties in orthogonal directions. This allows two-dimensional AO deflection with the same block of material.

AOD also serve another function in signal processing. The number of resolvable spots of an AOD scanner translates to the number of resolvable spectral components of the RF in AO spectrum analyzer applications and the number of corelations in AO corelator applications (Figure 11.47 and 11.48). 


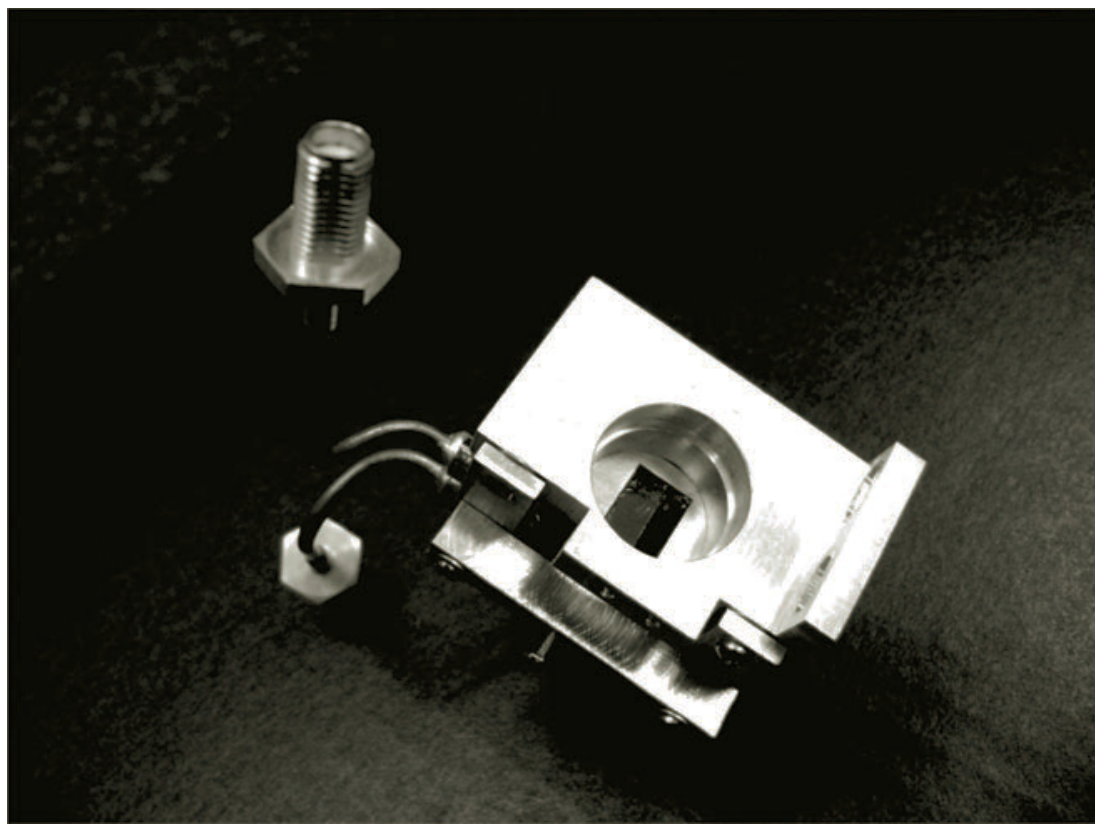

\section{FIGURE 11.46}

A typical two-channel GaP acousto-optic modulator for 1-GHz bandwidth modulation. (Courtesy MVM Electronics, Inc.)

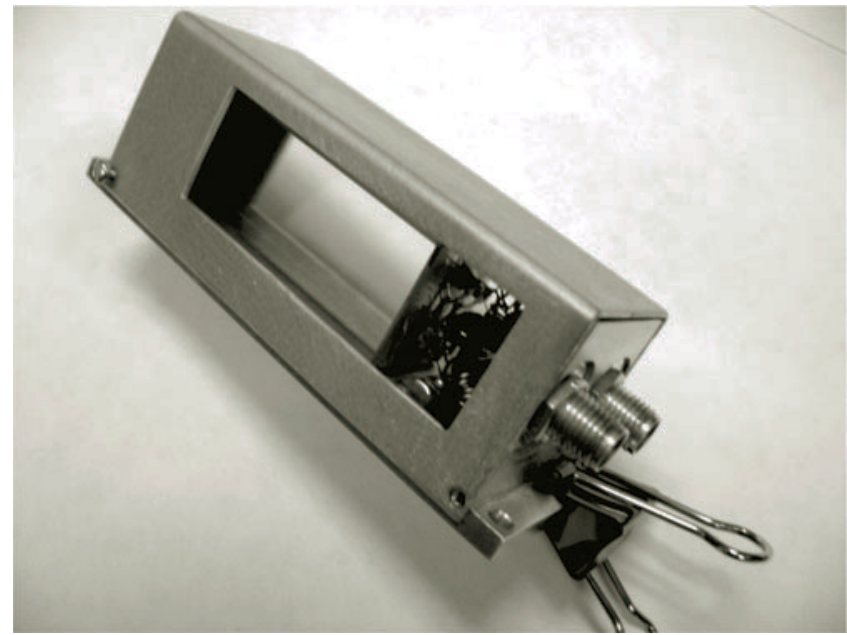

\section{FIGURE 11.47}

A typical 1000 resolvable spot acousto-optic deflector. (Courtesy MVM Electronics, Inc.)

\subsubsection{Acousto-Optic Frequency Shifters}

The traveling-wave interaction between light and acoustic waves imparts frequency shift to the diffracted light. Though AOFS generally require a narrow frequency band to function, some applications may desire a wide bandwidth. When the bandwidth is large, AOFS and AOM lose their distinction. If the frequency range is small, then the bandwidth of the piezoelectric transducer and the $\mathrm{AO}$ interaction can be traded off to achieve higher optical 


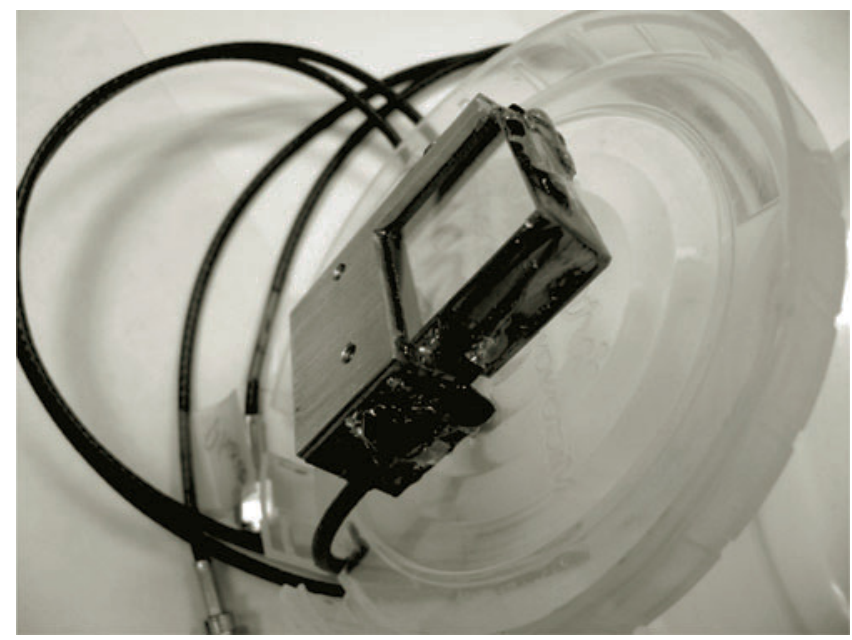

FIGURE 11.48

A typical two-dimensional acousto-optic deflector. (Courtesy MVM Electronics, Inc.)

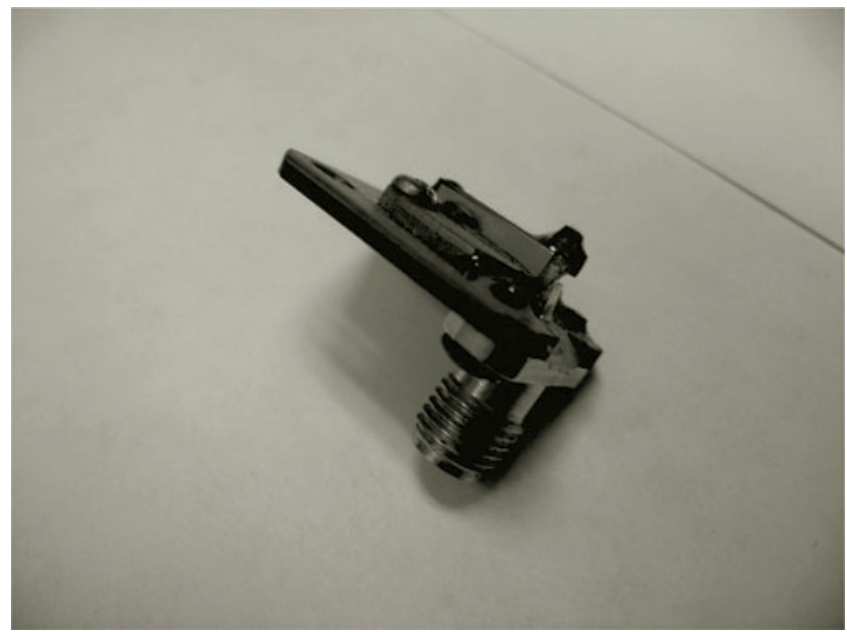

\section{FIGURE 11.49}

6.85-GHz acousto-optic frequency shifter. (Courtesy MVM Electronics, Inc.)

throughput at a much higher center frequency. AO frequency shift to $10 \mathrm{GHz}$ has been achieved, at least for low-percentage optical throughput (Figure 11.49).

\subsubsection{Acousto-Optic Tunable Filters}

The AO scheme is the only one available that allows electronically selectable imaging of narrow-band, multispectral optical components from a wide-spectrum light. In colinear propagating acoustic and optical wave devices, diffraction of one optical polarization to another can occur with Bragg matching k-vector supplied by the acoustic waves. Here, the interaction length can be very large resulting in narrow optical bandwidth diffraction. Using simultaneous multitone RF, many narrow optical bands can be diffracted. Moreover, 


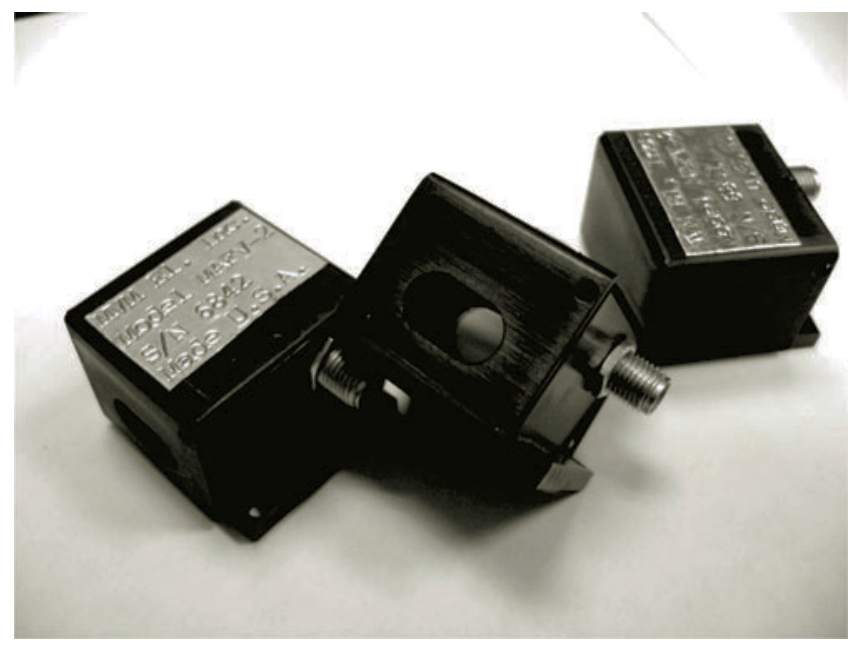

FIGURE 11.50

Tellurium dioxide acousto-optic tunable filter in the visible and infrared range. (Courtesy MVM Electronics, Inc.)

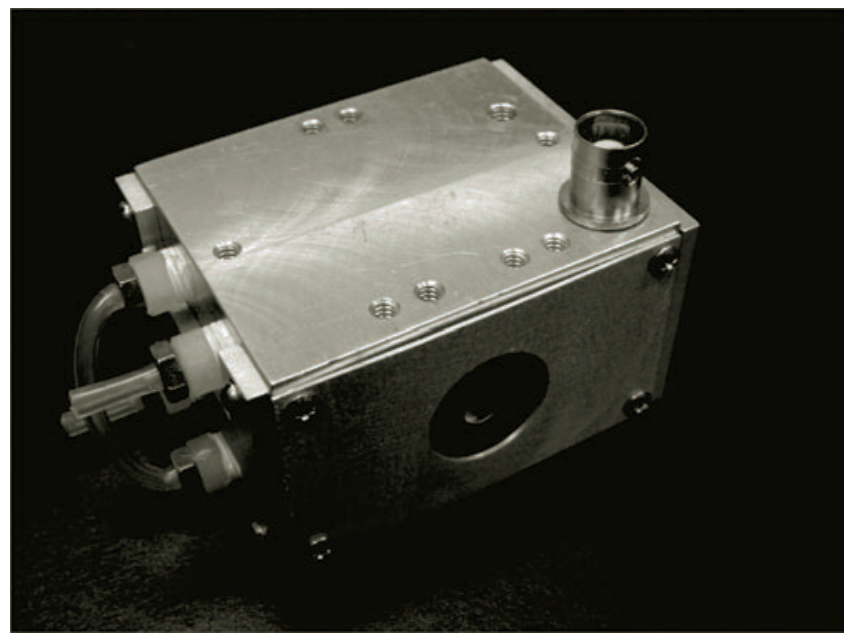

\section{FIGURE 11.51}

Crystalline quartz acousto-optic tunable filter in the ultraviolet range. (Courtesy MVM Electronics, Inc.)

the angular sensitivity of the AO interaction is small, resulting in good image preservation. One drawback of the colinear interaction is the difficulty of separating the diffracted and undiffracted components.

In certain materials such as tellurium dioxide and crystalline quartz, a so-called "parallel tangents Bragg matching condition" is possible. This condition allows orthogonal AO interaction and removes the difficulty of separating diffracted and undiffracted optical components (Figure 11.50 and 11.51).

The limitations of AOTF are set by the availability of large-size material, acoustic attenuation, transmission range of the selected material, and RF drive power requirements. 

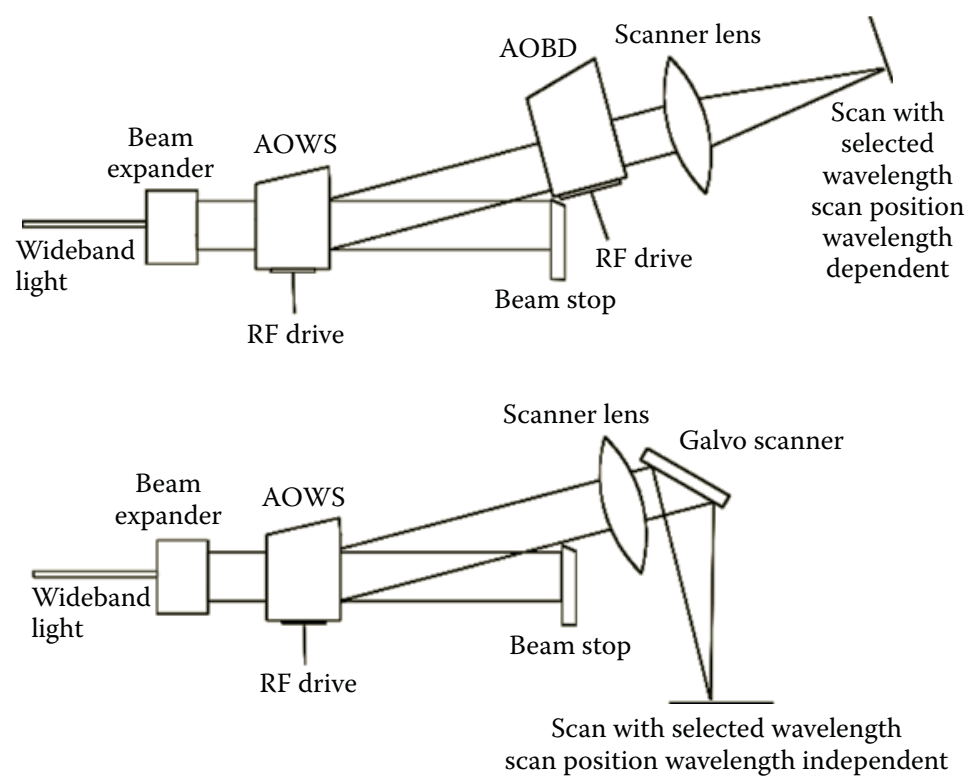

FIGURE 11.52

A schematic of a variable-wavelength optical scanner.

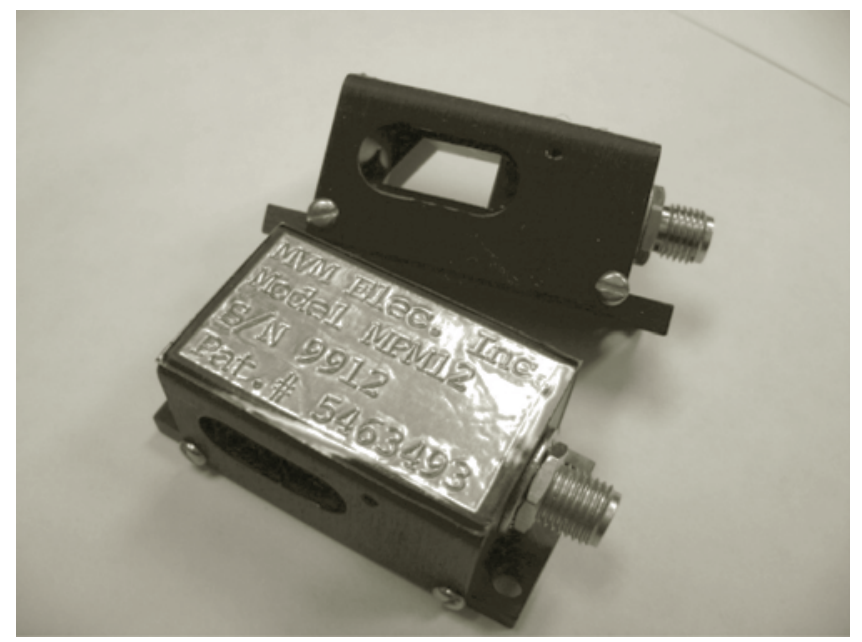

\section{FIGURE 11.53}

Device for full color argon and krypton laser projector systems used in entertainment and planetarium applications. (Courtesy MVM Electronics, Inc.)

\subsubsection{Acousto-Optic Wavelength Selectors}

In certain applications, the imaging function available in AOTF is not required. This relaxes several AOTF parameters that can be traded to reduce requirements for RF drive power and material size. In that case AOTF can function as AOWS. If a galvanometric or other mechanical scanner is placed after the AOWS, we could obtain a variable-wavelength optical scanner that may have wide-ranging applications (Figure 11.52). 


\subsubsection{Polychromatic Acousto-Optic Modulators}

If the diffraction efficiency of an AOWS can be improved further such that almost $100 \%$ diffraction can occur with relatively low RF drive power in the range of 20-100 $\mathrm{mW}$, then many RF tones approaching 16 can be used for the AOWS; this device is known as polychromatic AOM. An argonion laser contains six to ten wavelengths in the visible spectrum. Polychromatic AO modulation has been widely used for laser projection and laser shows to produce vivid, full-color images for entertainment and planetarium applications (Figure 11.53).

Material degradation at high laser power and linear polarization restriction is of concern in some applications.

\subsection{CONCLUSIONS}

AO devices have been utilized in a variety of scanning applications from direct spatial or temporal modulation to predeflection or postscan lensing. For most applications of these devices there are significant design trades between modulation bandwidth, efficiency, and other performance parameters. AOD are often advantageous in applications where high precision is required over a relatively small angular scan. AOM are effective for pixelation of raster scans or video modulation when it is impractical to directly modulate the optical source. The ability to modulate light without moving parts should continue to make acousto-optics an attractive technology in the future.

\section{ACKNOWLEDGMENTS}

We would like to make special mention to Dr. Manhar Shah of MVM Electronics for his valuable contribution on the applications of $\mathrm{AO}$ devices. We wish to thank Dr. Robert Montgomery for his review and assistance, and Damon Kvamme and Bryan Bolt for sharing their experiences with the application of $\mathrm{AO}$ devices.

\section{REFERENCES}

1. Debye, P.; Sears, F.W. Proc. Natl. Acad. Sci. 1932, 18, 409.

2. Lucas, R.; Biquard, P. J. Phys. Rad. 1932, 3(7), 464.

3. Raman, C.F.; Nath, N.S.N. Proc. Indian Acad. Sci. I 1935, 2, 406.

4. Gordon, E.I. Proc. IEEE 1966, 54, 1391.

5. Dixon, R.W. IEEE J. Quantum Electronics 1967, QE-3, 85.

6. Harris, S.E.; Nieh, S.T.R.; Winslow, D.K. Appl. Phys. Lett. 1969, 15, 325.

7. Mertens, R. Meded. K. Vlaam. Acad. Wet. Lett. Schone Künsten Relg., Kl. Wet. 1950, $12,1$.

8. Exterman, R.; Wannier, G. Helv. Phys. Acta 1936, 9, 520.

9. Klein, W.R.; Hiedemann, E.A. Physica 1963, 29, 981. 
10. Nomoto, O. Jpn. J. Appl. Phys. 1971, 10, 611.

11. Klein, W.R.; Cook, B.D. IEEE Trans. Sonics Ultrason. 1967, SU-14, 723.

12. Korpel, A. J. Opt. Soc. Am. 1979, 69, 678.

13. Korpel, A.; Poon, T. J. Opt. Soc. Am. 1980, 70, 817.

14. Chang, I.C. IEEE Trans. Sonics Ultrason. 1976, SU-23, 2.

15. Uchida, N.; Ohmachi, Y. J. Appl. Phys. 1969, 40, 4692.

16. Warner, A.W.; White, D.L.; Bonner, W.A. J. Appl. Phys. 1972, 43, 4489.

17. Cohen, M.; Gordon, E.I. Bell Syst. Tech. J. 1965, 44, 693.

18. Dixon, R.W. J. Appl. Phys. 1962, 38, 5149.

19. Young, E.H.; Yao, S.K. Proc. IEEE 1981, 69, 54.

20. Foster, L.C.; Crumly, C.B.; Cohoon, R.L. A high-resolution linear optical scanner using a traveling-wave acoustic lens. Appl. Opt. 1970, 9, 2154-2160.

21. Pinnow, D.A. IEEE J. Quantum Electronics 1970, QE-6, 223.

22. Gladstone, J.H.; Dale, T.P. Phil. Trans. Roy. Soc. London 1964, 153, 37.

23. Wemple, S.H.; DiDomenico, M. J. Appl. Phys. 1969, 40, 735.

24. Uchida, N.; Niizeki, N. Proc. IEEE 1973, 61, 1073.

25. Woodruff, T.O.; Ehrenreich, H. Phys. Rev. 1961, 123, 1553.

26. Mueller, H. Phys. Rev. 1935, 47, 947.

27. Abrams, R.L.; Pinnow, D.A. J. Appl. Phys. 1970, 41, 2765.

28. Dixon, R.W. J. Appl. Phys. 1967, 38, 5149.

29. Pinnow, D.A.; Van Uitert, L.G.; Warner, A.W.; Bonner, W.A. Appl. Phys. Lett. 1969, 15, 83.

30. Coquin, G.A.; Pinnow, D.A.; Warner, A.W. J. Appl. Phys. 1971, 42, 2162.

31. Ohmachi, Y.; Uchida, N. J. Appl. Phys. 1969, 40, 4692.

32. Gottlieb, M.; Isaacs, T.J.; Feichtner, J.D.; Roland, G.W. J. Appl. Phys. 1969, 40, 4692.

33. Roland, G.W.; Gottlieb, M.; Feichtner, J.D. Appl. Phys. Lett. 1972, 21, 52.

34. Isaacs, T.J.; Gottlieb, M.; Feichtner, J.D. Appl. Phys. Lett. 1974, 24, 107.

35. Feichtner, J.D.; Roland, G.W. Appl. Optics 1972, 11, 993.

36. Harris, S.E.; Wallace, R.W. J. Opt. Soc. Am. 1969, 59, 744.

37. Sittig, E.K. IEEE Trans. Sonics and Ultrasonics 1969, SU-16, 2.

38. Meitzler, A.H.; Sittig, E.K. J. Appl. Phys. 1969, 40, 4341.

39. Mason, W.P. Electromechanical Transducers and Wave Filters; Van Nostrand Reinhold: Princeton, NJ, 1948.

40. Sittig, E.K. IEEE Trans. Sonics and Ultrasonics 1969, 16, 2.

41. Meitzler, A.H. Ultrasonic Transducer Materials; Mattiat, O.E., Ed.; Plenum: New York, 1971.

42. deKlerk, J. Physical Acoustics; Mason, W.P., Ed.; Academic Press: New York, 1970; Vol. IV, Chap. 5.

43. deKlerk, J. IEEE Trans, on Sonics and Ultrasonics 1966, SU-13, 100.

44. Weinert, R.W.; deKlerk, J. IEEE Trans, on Sonics and Ultrasonics 1972, SU-19, 354.

45. Coquin, G.; Griffin, J.; Anderson, L. IEEE Trans, on Sonics and Ultrasonics 1971, SU-7, 34.

46. Korpel, A. et al. Proa IEEE 1966, 54, 1429.

47. Pinnow, D.A. IEEE Trans, on Sonics and Ultrasonics 1971, SU-18, 209.

48. Eschler, H. Optics Communications 1972, 6, 230.

49. Sittig, E.K.; Cook, H.D. Proc. IEEE 1968, 56, 1375.

50. Konog, W.F.; Lambert, L.B.; Schilling, D.L. IRE Int. Conv. Rec. 1961, 9 (6), 285.

51. Larson, J.D.; Winslow, D.K. IEEE Trans. Sonics and Ultrasonics 1971, SU-18, 142. 